\title{
A single bacterial sulfatase is required for metabolism of colonic mucin 0-glycans and intestinal colonization by a symbiotic human gut bacterium
}

\section{Eric Martens ( $\square$ emartens@umich.edu )}

University of Michigan-Ann Arbor https://orcid.org/0000-0001-6681-2990

Ana Luis

University of Michigan; University of Gothenburg

Chunsheng Jin

Gothenburg University https://orcid.org/0000-0002-0229-102X

Gabriel Pereira

University of Michigan Medical School https://orcid.org/0000-0001-7937-474X

Robert Glowacki

University of Michigan

\section{Sadie Gugel}

University of Michigan

Shaleni Singh

University of Michigan

Dominic Byme

University of Liverpool

Nicholas Pudlo

University of Michigan

James London

University of Liverpool

Arnaud Basle

Newcastle University

Mark Reihill

University College Dublin

\section{Stefan Oscarson}

University College Dublin

\section{Patrick Eyers}

University of Liverpool https://orcid.org/0000-0002-9220-2966

\section{Mirjam Czjzek}

CNRS, Station Biologique de Roscoff 


\section{Gurvan Michel}

CNRS, Station Biologique de Roscoff

\section{Tristan Barbeyron}

Station Biologique de Roscoff

\section{Edwin Yates}

University of Liverpool

\section{Gunnar Hansson}

University of Gothenburg https://orcid.org/0000-0002-1900-1869

\section{Niclas Karlsson}

University of Gothenburg

Alan Cartmell

University of Liverpool

\section{Biological Sciences - Article}

Keywords: colonic mucin O-glycans, symbiotic human gut bacterium, intestinal colonization

Posted Date: January 12th, 2021

DOl: https://doi.org/10.21203/rs.3.rs-113032/v1

License: (c) (1) This work is licensed under a Creative Commons Attribution 4.0 International License. Read Full License

Version of Record: A version of this preprint was published at Nature on October 6th, 2021. See the published version at https://doi.org/10.1038/s41586-021-03967-5. 
2 A single bacterial sulfatase is required for metabolism of colonic mucin 0 -glycans 3 and intestinal colonization by a symbiotic human gut bacterium

${ }^{* 1,2}$ Ana S. Luis, ${ }^{2}$ Chunsheng Jin, ${ }^{1}$ Gabriel Vasconcelos Pereira, ${ }^{1}$ Robert W. P. Glowacki, ${ }^{1}$ Sadie Gugel, ${ }^{1}$ Shaleni Singh, ${ }^{3}$ Dominic P. Byrne, ${ }^{1}$ Nicholas Pudlo, ${ }^{3}$ James A London, ${ }^{4}$ Arnaud Baslé, ${ }^{5}$ Mark Reihill, ${ }^{5}$ Stefan Oscarson, ${ }^{3}$ Patrick A. Eyers, ${ }^{6}$ Mirjam, Czjzek, ${ }^{6}$ Gurvan Michel, ${ }^{6}$ Tristan Barbeyron, ${ }^{3}$ Edwin A Yates, ${ }^{2}$ Gunnar C. Hansson, ${ }^{2}$ Niclas G. Karlsson, ${ }^{*}, 3$ Alan Cartmell, ${ }^{* 1}$ Eric C. Martens

${ }^{1}$ Department of Microbiology and Immunology, University of Michigan, Ann Arbor, MI 14 48109, USA

152 Department of Medical Biochemistry, Institute for Biomedicine, Sahlgrenska Academy,

16 University of Gothenburg, Box 440, 40530 Gothenburg, Sweden

$17{ }^{3}$ Department of Biochemistry and Systems Biology, Institute of Systems, Molecular and

18 Integrative Biology, University of Liverpool, Liverpool L69 3BX, United Kingdom

$19{ }^{4}$ Institute for Cell and Molecular Biosciences, Newcastle University, Newcastle upon

20 Tyne, United Kingdom

$21{ }^{5}$ Centre for Synthesis and Chemical Biology, University College Dublin, Belfield, Dublin 22 4, Ireland.

23 '5orbonne Université, Univ Paris 06, CNRS, UMR 8227, Integrative Biology of Marine 24 Models, Station Biologique de Roscoff, CS 90074, Roscoff, Bretagne, France.

${ }^{*}$ Correspondence to:

emartens@umich.edu

Alan.Cartmell@liverpool.ac.uk ana.luis@medkem.gu.se 


\section{Summary}

Humans have co-evolved with a dense community of microbial symbionts that inhabit the lower intestine. In the colon, secreted mucus creates a physical barrier that separates these microbes from the intestinal epithelium. Some gut bacteria are able to utilize mucin glycoproteins, the main mucus component, as a nutrient source. However, it remains unclear which bacterial enzymes initiate the degradation of the highly complex O-glycans found in mucins. In the colon, these glycans are heavily sulfated, but the specific sulfatases that are active on colonic mucins have not been identified. Here, we show that sulfatases are essential to the utilization of colonic mucin O-glycans by the human gut symbiont Bacteroides thetaiotaomicron. We have characterized the activity of 12 different sulfatases encoded by this species, showing that these enzymes collectively are active on all of the known sulfate linkages in colonic O-glycans. Crystal structures of 3 enzymes provide mechanistic insight into the molecular basis of substrate-specificity. Unexpectedly, we found that a single sulfatase is essential for utilization of sulfated $O$ glycans in vitro and also plays a major role in vivo. Our results provide insight into the mechanisms of mucin degradation by gut bacteria, an important process for both normal microbial gut colonization and diseases such as inflammatory bowel disease (IBD). Sulfatase activity is likely to be a keystone step in bacterial mucin degradation and inhibition of these enzymes may therefore represent a viable therapeutic path for treatment of IBD and other diseases.

\section{Introduction} health and disease, including inflammatory bowel disease (IBD) ${ }^{1}$ and colorectal cancer $(\mathrm{CRC})^{2}$. In the colon, secreted mucus creates a physical barrier that separates gut microbes from the intestinal epithelium ${ }^{3}$ preventing close contact that can lead to inflammation and eventual CRC if this barrier is either experimentally eliminated ${ }^{4,5}$ or has reduced glycosylation ${ }^{6-9}$. A major component of the colonic mucus is mucin 2 (MUC2), a glycoprotein that contains up to $80 \%$ glycans by mass and more than 100 different glycan structures that are 0 -linked to serine or threonine residues ${ }^{10}$. Mucin glycosylation is variable along the gastrointestinal $(\mathrm{Gl})$ tract with a marked increase in sulfation in the 
65 colon $^{11}$. In mucins, O-linked sulfate may be attached to the 6-hydroxyl of $\mathrm{N}$-acetyl-D66 glucosamine (6S-GlcNAc) and non-reducing end D-galactose (Gal) sugars at hydroxyl 67 positions 3-, 4- or 6- (3S-, 4S- and 6S-Gal, respectively) ${ }^{11-13}$ (Fig. 1a). Sulfation often occurs as terminal caps that block enzymatic degradation of oligosaccharides. To degrade and utilize colonic mucin O-glycans, members of the HGM need to express appropriate carbohydrate sulfatases to remove these modifications. Bacteroides thetaiotaomicron $(B t)$ is a dominant member of the human gut microbiota that is able to utilize O-glycans as a sole nutrient source ${ }^{14}$. Underscoring the importance of sulfatases, $B t$ requires active sulfatases for competitive colonization of the wild-type mouse gut ${ }^{15}$ and to induce inflammation in genetically-susceptible mice ${ }^{16}$. However, the specific sulfatases that mediate these effects remain unknown. Indeed, despite the critical roles of sulfatases in many biological processes, including several human diseases ${ }^{17}$, a significant knowledge gap exists regarding the biochemical, structural and functional roles of these enzymes.

We hypothesized that specific $B t$ sulfatases play essential roles in initiation of $O$ glycan degradation. To test this, we measured the activities of 23 putative $B t$ sulfatases, determining that 12 enzymes are active on either model glycan substrates or purified colonic mucin O-glycans. Together with defining the specific activities of these enzymes, we determined the corresponding structures for 3 sulfatases, revealing the basis of substrate specificity. Using molecular genetics, we next assessed the contributions of these sulfatases to $B t$ fitness in vitro and in vivo, unexpectedly revealing that a single enzyme is essential for utilization of sulfated mucin O-glycans and plays a major role in competitive gut colonization. Identifying specific bacterial sulfatases that are critical for intestinal mucin degradation provides new potential targets, with a goal of blocking progression of diseases such as IBD and possibly other disorders that result from bacterial disruption of the mucus barrier.

\section{Utilization of colonic mucins by HGM species}

Several studies have identified HGM members that are able to utilize porcine gastric mucin O-glycans (gMO) as a sole carbon source ${ }^{14,18,19}$. However, this substrate 
96 colon, especially those with increased sulfation that are lacking in $\mathrm{gMO}^{11}$. To identify HGM

97 species that utilize sulfated colonic mucins, we measured the growth of 19 Bacteroides

98 type strains, plus Akkermansia muciniphila, on highly sulfated porcine colonic mucin 99 oligosaccharides (cMO). We identified six Bacteroides strains that utilize cMO (Fig. 1b,

100 Extended Data Fig. 1). Interestingly, two known mucin-degraders, A. muciniphila ${ }^{20}$ and

101 B. massiliensis ${ }^{21}$, grew robustly on gMO but failed to utilize sulfated $\mathrm{CMO}$ as a substrate

102 (Extended Data Fig. 1), highlighting the importance of employing colonic mucins as 103 substrates to draw more physiologically-relevant conclusions about the metabolic targets 104 of these organisms. $B t$, the bacterium with the highest number of sulfatases (28), was 105 one of the strains with the best growth on $\mathrm{CMO}$ (Fig. 1 b), suggesting that some of these 106 enzymes might play key roles in promoting this ability. Therefore, to understand the role 107 of sulfatases in colonic mucin utilization by HGM bacteria we focused on the biochemical 108 and genetic characterization of the Bt enzymes. See Supplementary Discussion 1 for additional details of $\mathrm{GI}$ mucins and bacterial growth kinetics.

\section{Substrate specificity of Bt sulfatases}

Sulfatases are classified into four main families (S1 to S4) in the SulfAtlas database according to sequence similarity, catalytic mechanism and fold ${ }^{22}$. Family $S 1$ is currently divided into 72 subfamilies (designated S1_X) and comprises the formylglycine sulfatases, which operate via a hydrolytic mechanism that utilizes a non-genetically coded formylglycine amino acid as its catalytic residue. In $B t$ and other anaerobic bacteria, this

117 residue is introduced co-translationally by the anaerobic sulfatase maturating enzyme 118 (anSME) $)^{15}$, which converts a serine or cysteine, within the consensus sequence $\mathbf{C} / \mathbf{S}$-X$119 \mathrm{P} / \mathrm{A} / \mathrm{S}-\mathrm{X}-\mathrm{R}$, to formylglycine, which then serves as catalytic nucleophile ${ }^{23}$. The $B t$ genome 120 encodes 28 S1 sulfatases classified into twelve different subfamilies (Supplementary 121 Table 1). Four $B t$ sulfatases have been previously characterized and all act on 122 glycosaminoglycans (GAGs) that are components of extracellular matrix (Fig. 1c) ${ }^{24,25}$. 123 Interestingly, several of the uncharacterized S1 sulfatases are encoded within 124 polysaccharide utilization loci (PULs) that are known to be upregulated in vivo or during 125 growth on $\mathrm{gMO}^{14}$ and encode other glycoside hydrolases enzymes potentially involved in 126 degrading mucin O-glycans (Extended Data Fig. 2). 
To understand the role of sulfatases in mucin metabolism, we cloned and

128 expressed in soluble form 23 of the remaining 24 uncharacterized sulfatases. The 129 recombinant proteins were tested for activity against a panel of commercially available 130 sulfated saccharides (Supplementary Table 2). In this initial screen, we identified 131 activities for twelve sulfatases (Fig. 1c, Extended Data Figs. 3, 4 and Supplementary 132 Table 3). Among these, 5 of the enzymes represent the first activities reported for their 133 respective subfamilies: two S1_20 members (BT1636 and BT1622) were determined to 134 target 3S-Gal, with BT1622 also preferentially cleaving 3S-N-acetyl-D-galactosamine (3S135 GalNAc), two S1_16 enzymes (BT3796 and BT3057) cleave 4S-Gal/4S-GalNAc and one 136 S1_46 enzyme (BT1918) cleaves 3S-GlcNAc, using the $N$-acetyl group as an absolute 137 specificity determinant. This represents the first report of a bacterial sulfatase active on 138 3S-GaINAc, indicating that this sulfation could exist as a yet unidentified modification of 139 host glycans. Subsequently, we refer to these enzymes by their gene/locus tag number with the corresponding activity in superscript (e.g., BT1636 3 -Gal).

141 In addition to assigning new catalytic activities associated with three subfamilies 142 that previously lacked any characterization, we also identified sulfatases displaying novel 143 activities inside previously characterized subfamilies. These include three S1_15 144 enzymes (BT16246S-Gal/GalNAc, BT31096S-Gal/GalNAc and BT46316S-Gal/GalNAc) that extend this 145 family, previously only known to include 6S-GalNAc sulfatases, to those cleaving 6S-Gal. 146 Two members of S1_4 were active on 3S-Gal (BT4683 ${ }^{3 S-G a l}$ ) or 6S-Gal (BT3487 ${ }^{6 S-G a l}$ ), 147 representing novel activities within this arylsulfatase subfamily. Finally, consistent with 148 the activity previously described for S1_11 members, two enzymes were 6S-GIcNAc 149 sulfatases (BT1628 ${ }^{6 S-G I c N A c}$ and BT3177 ${ }^{6 S-G l c N A c}$ ) (Fig. 1c and Extended Data Fig. 3).

150 Characterized sulfatases within the same subfamily, with the exception of two 151 S1_4 members, cleaved the same sulfate ester linkages (Fig. 1c). However, despite 152 these enzymes targeting the same linkages, their optimal activity depends on the 153 surrounding glycan context. The activity of the 3S-Gal sulfatases is dependent on the 154 linkage between Gal and GlcNAc with BT468335-Gal showing a preference for 3'-sulfate$155 N$-acetyl-D-lactosamine (3'S-LacNAc, 3'S-D-Gal- $\beta 1,4-D-G I c N A c)$. In contrast, BT1622 ${ }^{3 S-}$ 156 Gal/GalNAc demonstrated enhanced activity towards 3'-sulfate-lacto-N-biose (3'S-LNB, 3'S157 D-Gal- $\beta 1,3-D-G l c N A c)$ (Extended Data Fig. 4a and Supplementary Table 3). 
158 Furthermore, additional affinity/activity studies revealed that BT1622 ${ }^{3 S-G a l / G a l N A c}$ 159 preferentially targets GalNAc and not Gal (Extended data Fig. 4b,c and Supplementary

160 Table 3), suggesting that this sulfatase evolved to optimally target sulfate O3-linked to 161 GalNAc. Bt sulfatase activity is also affected by the presence of terminal epitopes such 162 as those that occur in Lewis antigens. Despite BT1636 3 -Gal being equally active on 3'S163 LacNAc and 3'S-LNB, this protein has a lower affinity and it is 100-fold less active when 164 L-fucose (Fuc) is linked to GlcNAc (3'S-Lewis-a/x) (Extended data Fig. 4a,b and 165 Supplementary Table 3). While BT1622 ${ }^{3 S-G a l / G a l N A c}$ is only weakly active on 3'S-Lewis-a 166 antigen and not active at all on 3'S-Lewis-x, the reciprocal is true for BT4683 ${ }^{3 S-G a l}$ 167 (Supplementary Table 3). The subfamily S1_15 enzyme BT1624 ${ }^{6 S-G a l / G a l N A c}$ was only 168 weakly active on 6S-Lewis-a/x antigens (Extended Data Fig. 4a), suggesting that these 169 enzymes cannot accommodate Fuc linked to GIcNAc and that Fuc needs to be removed 170 prior to sulfate cleavage. Additionally, affinity studies showed that BT3109 ${ }^{6 S-G a l / G a l N A c}$ has 171 a strong affinity for Gal, while the previously characterized GAG sulfatase BT3333 ${ }^{6 \mathrm{~S}-G a l N A c}$ 172 showed a preference for GalNAc, suggesting that optimal activity of S1_15 sulfatases 173 likely depends on the glycan context and BT33336S-GalNAc evolved to target sulfated 174 linkages in GAGs, a substrate that contains GalNAc but not Gal.

\section{Bt sulfatase activity on colonic mucin oligosaccharides (cMO)}

We next tested the activity of $B t$ sulfatases on custom purified $\mathrm{cMO}$, which we determined to contain at least 131 different oligosaccharides (Supplementary Table 4). Only 4 of the 6 sulfatases tested displayed activity on cMOs (Fig. 2a,b and Supplementary Table 4). BT1628 ${ }^{6 S-G I C N A C}$ and BT3177 ${ }^{6 S-G I C N A c}$ removed 6-O-sulfate from all GIcNAc structures but only when present at the non-reducing end of O-glycans confirming an exo-mode of action observed using commercial oligosaccharides (Fig. 2 and Extended Data Fig. 5a). After incubation with BT1636 $36^{3 \mathrm{~S}-G a l}$, we were able to detect 14 new oligosaccharides and an overall increase of non-sulfated glycans (Fig. 2 and Extended Data Fig. 5b). Compared to the non-enzyme treated control, 36 oligosaccharides could no longer be detected after incubation with BT1636 ${ }^{3 S-G a l}$ (Fig. 2a and Supplementary Table 4). We determined the structures of 8 of these glycans and 
189 (core 1) and more complex sulfated structures built around other common core structures

190 (Fig. 2c), indicating that BT1636 ${ }^{3 S-G a l}$ evolved to accommodate the various linkages and 191 substitutions found in mucin O-glycans. After incubation with BT4683 ${ }^{3 S-G a l}, 9$

192 oligosaccharides disappeared, indicating that this enzyme is active on a smaller subset

193 of structures (Fig. 2a and Supplementary Table 4). We were only able to determine the 194 structure of one of those glycans and, unexpectedly, we found that this enzyme is endo195 active on sialylated 3S-Gal (Fig. 2b), which is consistent with the protein structural data discussed below. Additionally, the strong activity of BT1636 $3 \mathrm{~S}-\mathrm{Gal}$ on larger glycans with unknown structures (Supplementary Table 4) reveals that porcine cMOs are highly sulfated at the O3-position of galactose. Although previous studies have reported the presence of $3 S-G a l$ in colonic mucins ${ }^{26-28}$, the analysis of such complex samples is technically challenging and, until now, no precise enzymatic tools were available to probe these linkages. Together, these results represent the first report of HGM sulfatases active on colonic mucin O-glycans and highlight the possibility to use gut bacterial sulfatases as analytical tools in structural characterization of mucin glycans (see Supplementary Discussion 2 for additional details of sulfatase activity on cMO).

\section{Structural characterization of 3S-Gal/GaINAc sulfatases}

To further understand the molecular details of carbohydrate recognition by S1 sulfatases, we determined the crystal structures of the three different 3S-Gal sulfatases that belong to two subfamilies. Consistent with previous structures of S1 sulfatases, all 3 enzymes display a $\alpha / \beta / \alpha$ topology with a C-terminal sub-domain, and the active site residues interacting with the sulfate group are fully conserved (Extended data Fig. 6a,b).

212 The structure of BT1636 ${ }^{3 \mathrm{~S}-G a l}$ in complex with the product LacNAc revealed that His177

213 coordinates with $\mathrm{O} 4$ of $\mathrm{Gal}$ and mutation to alanine ablates enzyme activity, suggesting

214 that this residue is the major specificity determinant for Gal (presenting an axial O4) over

215 glucose (equatorial O4) (Fig. 3 and Supplementary Table 3). BT1636 35 -Gal also makes

216 strong interactions with O2 via R353 and E334. The essential His177 in BT1636 ${ }^{3 S-G a l}$ is

217 highly conserved (92\%) within S1_20 sulfatases (Extended data Fig. 7). In BT1622 ${ }^{3 S-}$

$218 \mathrm{Gal} / \mathrm{GalNAc}$, mutation of the corresponding $\mathrm{H} 176$ to alanine causes a $\sim 300$-fold reduction in

219 activity (Supplementary Table 3), further highlighting the importance of this residue in 
220 Gal recognition. In BT1622 3 S-Gal/GalNAc, R353 and E334 are replaced by C357 and N334,

221 amino acids that present shorter side chains that allow the accommodation of a C2-linked

$222 \mathrm{~N}$-acetyl group found in GalNAc (Fig. 3). This observation is consistent with the ability of

223 BT16223S-Gal/GalNAc to cleave 3S-GalNAc and preferentially bind GalNAc over Gal, while

224 BT1636 ${ }^{3 S-G a l}$ only recognizes Gal (Extended Data Fig. 4b,c). Additionally, and consistent

225 with exo-activity observed for both enzymes, the substrates are buried in a deep pocket

226 and only 01 is solvent exposed (Fig. 3).

227 For the S1_4 enzyme BT4683 3 SS-Gal, a structure solved in complex with LacNAc did 228 not reveal any interaction between the protein and the $\mathrm{O} 4$ of Gal. In BT4683 ${ }^{3 \mathrm{~S}-G a l}$, the 229 interaction with $\mathrm{Gal}$ is driven by the residues R72 and E335, spatially equivalent to R353 230 and E334 in BT16363s-Gal, that form hydrogen bonds with O2 of D-Gal (Fig. 3) and 231 disruption of either of these residues eliminates activity (Supplementary Table 3). 232 However, in BT4683 ${ }^{3 \mathrm{~S}-G a l}$, a sulfatase that does not have any affinity for monosaccharides

233 (Extended data Fig. 4b), the active site is located in an open cleft (Fig. 3) that allows the 234 accommodation of additional substitutions on Gal (Extended Data Fig. 6d). This finding 235 is consistent with the apparent endo-activity found using cMO. Together, these structures 236 reveal the key specificity determinants in 3S-Gal/GalNAc sulfatases, highlighting that 237 these enzymes have evolved to target sulfate groups in the different contexts in which 238 they are found in complex host glycans. This is especially true for BT1636 35 -Gal which 239 utilizes high affinity interactions with both $\mathrm{O} 2$ and $\mathrm{O} 4$ to drive enhanced activity to remove 240 terminal 3S-Gal linkages in cMOs (see Supplementary Discussion 3 for additional 241 details of sulfatase structural characterization and phylogeny).

\section{Roles of sulfatases in B. thetaiotaomicron O-glycan utilization}

$244 B t$ is able to utilize cMO as a sole carbon source (Fig. 1 b), but the key enzymes 245 involved in the degradation of these glycans remain unclear. Highlighting the importance 246 of sulfatases to this symbiont's physiology, deletion of the gene encoding the only 247 anaerobic sulfatase maturating enzyme (anSME) eliminates activation of all 28 S1 248 sulfatases ${ }^{15}$ and the ability of $B t$ to grow efficiently on cMOs (Fig. 4a). Based on this 249 observation we generated a series of strains with compounded gene deletions in which 250 one or several groups of sulfatases were eliminated based on their biochemical activity. 
251 Deletion of all 3S-Gal/GalNAc sulfatases $\left(\Delta b t 1636^{3 S-G a l}+\Delta b t 1622^{3 S-G a l / G a l N A c}+\Delta b t 4683^{3 S-}\right.$

$252 \mathrm{Gal}$ ) resulted in a growth phenotype similar to $\triangle a n S M E$ (Extended Data Fig. 8a). 253 Interestingly, we observed a similar growth defect when just BT1636 ${ }^{3 \mathrm{~S}-G a l}$ was deleted, 254 but not the other 3S-Gal sulfatases (Fig. 4a and Extended Data Fig. 8a), consistent with 255 the prominent activity of the recombinant form of this enzyme on cMOs. In contrast, a 256 strain with compounded deletions of eight other sulfatases $\left(\triangle b t 1622^{3 S-G a l / G a l N A c}+\right.$ $257 \Delta b t 4683^{3 S-G a l}+\Delta b t 1624^{6 S-G a l / G a l N A c}+\Delta b t 3109^{6 S-G a l}+\Delta b t 4631^{6 S-G a l / G a l N A c}+\Delta b t 1628^{6 S-G l c N A c}$

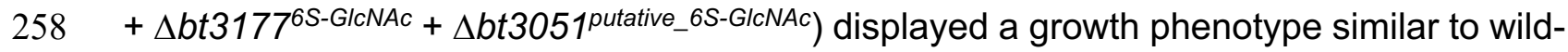
259 type (Fig. 4a), indicating that these enzymes are not essential for cMO utilization. 260 However, a $\Delta 10 X$ sulf mutant, which included the deletion of BT1636 $35-$ Gal and the two 261 4S-Gal/GalNAc sulfatases, showed a similar growth defect as $\triangle a n S M E$ and $\triangle b t 1636$. 262 Complementation of this and other loss of function mutants with only bt1636 ${ }^{3 S-G a l}$ restored 263 growth on cMO to levels similar to wild-type (Fig. 4a and Extended Data Fig. 8a). Cellular 264 localization experiments revealed that BT1636 $35-G a l$ is located at the cell surface in Bt 265 (Fig. 4b) and together these data suggest that this single 3S-Gal sulfatase is a critical cell 266 surface enzyme involved in the utilization of sulfated O-glycans that are prominent in the 267 colon.

268 To further investigate the role of BT1636 3 -Gal in O-glycan utilization, we analyzed 269 the oligosaccharides present in the culture supernatant of the wild-type and $\Delta b t 1636^{3 S-G a l}$ 270 strains after growth on cMO. Consistent with a robust ability of $B t$ to degrade diverse 271 colonic O-glycans, no oligosaccharides were detected in wild-type supernatant 272 (Supplementary Table 5). Compared to the cMO used as substrate (control), the 273 supernatant of $\Delta b t 1636^{3 S-G a l}$ showed a 20 -fold accumulation of terminal 3S-Gal capped 274 glycans, suggesting that these could not be degraded. Indeed, the three most common 275 3S-Gal structures detected in the $\Delta b t 1636^{3 S-G a l}$ supernatant accounted for $50 \%$ of the 276 total oligosaccharides detected in this sample (Fig. 4c). These results reveal that deletion 277 of BT1636 3 S-Gal results in loss of the ability to utilize 3S-Gal O-glycans. Interestingly, 49 278 of the 72 glycans detected in $\Delta b t 1636^{3 S-G a l}$ supernatant were not present in the control 279 sample (Supplementary Table 5), suggesting that although the mutant does not grow 280 well on cMO it is able to modify these oligosaccharides to generate new glycans 
281 (Supplementary discussion 5). These data, combined with the cell surface location of

282 BT1636 ${ }^{3 S-G a l}$, suggest that this sulfatase is required early in O-glycan catabolism likely by

283 cleaving 3S-Gal from O-glycans prior to importing them into the periplasm where these

284 oligosaccharides will be sequentially degraded by additional enzymes and also serve as

285 cues for activating transcription of the O-glycan PULs (Extended Data Fig. 2). Although

286 Bt does encode two additional 3S-Gal/GalNAc sulfatases, the low activity of these

287 additional sulfatases on cMOs (Fig. 2) and their likely periplasmic location, suggests why

288 these enzymes cannot compensate for loss of BT1636 ${ }^{3 S-G a l}$. Interestingly, all of the

289 Bacteroides species able to utilize cMO (Fig. 1a) have homologues of BT1636 ${ }^{3 S-G a l}$,

290 suggesting that this activity plays a key role in mucin utilization by other HGM members

291 (Supplementary Table 6).

Finally, to investigate the requirement for specific sulfatases in vivo, we utilized gnotobiotic mice in which we competed individual mutants against the wild-type strain to evaluate their colonization fitness. It has been reported that mouse colonic Muc2 prominently displays 6S-GIcNAc modifications ${ }^{29}$. However, mutants lacking either just the two active $6 S-G I c N A c$ sulfatases ( $\triangle 6 S-G / c N A c$ double mutant), or these two enzymes, another putative $6 \mathrm{~S}-$ GIcNAc sulfatase and all three $6 \mathrm{~S}-\mathrm{Gal} / \mathrm{GalNAc}$ sulfatases ( $\triangle 6 \mathrm{~S}$ GlcNAc+ $\triangle 6 S-G a l / G a l N A c$ hexa mutant), competed equally with wild-type (Extended Data Fig. 8c), suggesting that neither of these two sulfatase activities are essential determinants in vivo. A significant defect was observed with a mutant lacking all 3SGal/GalNAc sulfatases (Fig. 4d). The fitness defect was exacerbated by eliminating 3SGal/GalNAc and 6S-GIcNAc sulfatase activities together ( $\triangle 3 S-G a l / G a l N A c+\Delta 6 S$ GIcNAc) (Fig. 4d), suggesting that they synergize in vivo. Consistent with its prominent role in cMO utilization in vitro, a mutant lacking just BT1636 Gal-3Sulf displayed a significant defect that was similar or slightly more severe compared to the $\triangle 3 S$-Gal/GalNAc mutant when competed with the wild-type strain (Fig. 4d), further suggesting that this enzyme plays an essential role in gut colonization by allowing $B t$ to access 3S-Gal O-glycans (see Supplementary Discussion 4-5 for additional details regarding growth and competition of mutants in vitro and in vivo). 


\section{Conclusion}

313 To degrade the complex O-glycans found in mucins some HGM bacteria have 314 evolved complex arsenals of degradative enzymes which include diverse sulfatases.

315 Disarming all of the sulfatases in Bt via anSME deletion results in drastically reduced 316 competitive colonization (Extended Data Fig. 8c) ${ }^{15}$ and an inability to elicit colitis in an 317 animal model of IBD ${ }^{16}$. While these findings support a critical role for active sulfatases in 318 both fitness and promoting inflammation, they provide no insight into the complexity of 319 catalytic modifications carried out by these enzymes. In this study, we reveal that $B t$ has 320 a robust ability to grow on highly sulfated mucin oligosaccharides from colonic tissue and 321 that it possesses active sulfatases capable of removing sulfate groups in all contexts in

322 which sulfation is known to occur in mucin, including novel specificities. Surprisingly, we 323 found that a single key sulfatase is essential for growth on colonic mucin O-glycans. This 324 cell surface enzyme removes 3-sulfate capping Gal allowing the degradation of these 325 glycans by additional enzymes. This critical role of BT1636 35 -Gal supports the conclusion 326 that keystone steps exist in the complex pathway of mucin degradation. Further 327 delineation of these critical steps, along with identification of the corresponding 328 enzymes(s), are a prerequisite to modulating such events and potentially inhibiting mucin329 degrading activities in bacteria that contribute to disease.

\section{Methods}

\section{Recombinant Protein Production}

335 Genes were amplified by PCR using the appropriate primers and the amplified DNA

336 cloned in pET28b using Nhel/Xhol restriction sites or pETite (Expresso ${ }^{\mathrm{TM}} \mathrm{T7}$ cloning and 337 expression system, Lucigen) generating constructs with either $\mathrm{N}$ - or C-terminal His 6 tags 338 (Supplementary Table 7). The catalytic serine was mutated to cysteine since 339 Escherichia coli in only able to convert cysteine to formylglycine. Recombinant genes 340 were expressed in Escherichia coli strains BL21 (DE3) or TUNER (Novagen), containing 341 the appropriate recombinant plasmid, and cultured to mid-exponential phase before 342 induction with $1 \mathrm{mM}(\mathrm{BL21(DE3))}$ or $0.2 \mathrm{mM}$ (TUNER) of isopropyl $\beta$-D-1- 
343 thiogalactopyranoside; cells were cultured for another $16 \mathrm{~h}$ at $16^{\circ} \mathrm{C}$ and $180 \mathrm{rpm}$.

344 Recombinant protein were purified to $>90 \%$ electrophoretic purity by immobilized metal 345 ion affinity chromatography using a cobalt-based matrix (Talon, Clontech) and eluted with 346 imidazole as described previously ${ }^{24}$. For the proteins selected for structural studies,

347 another step of size exclusion chromatography was performed using a Superdex 16/60

348 S200 column (GE Healthcare), with $10 \mathrm{mM} \mathrm{HEPES,} \mathrm{pH} \mathrm{7.5,} \mathrm{and} 150 \mathrm{mM} \mathrm{NaCl}$ as the 349 eluent, and they were judged to be $\geq 95 \%$ pure by SDS-PAGE. Protein concentrations 350 were determined by measuring absorbance at $280 \mathrm{~nm}$ using the respective molar 351 extinction coefficient. When necessary, proteins were then concentrated by centrifugaton 352 using a molecular mass cutoff of $30 \mathrm{kDa}$.

353

\section{Site-Directed Mutagenesis}

355 Site-directed mutagenesis was conducted using the PCR-based QuikChange kit 356 (Stratagene) and conducted according to the manufacturer's instructions, using the 357 appropriate plasmid as the template and primers (Supplementary Table 8). All mutations 358 were confirmed by DNA sequencing.

360 Sources of purified carbohydrates

361 All carbohydrates were from Sigma, Carbosynth or Dextra Laboratories. All other 362 chemical reagents were purchased from Sigma. The 3S-GalNAc was chemically 363 synthesized as previously described ${ }^{30}$.

\section{Mucin purification}

366 Gastric mucin oligosaccharides (gMO) were purified from commercial available porcine gastric mucins (type III, Sigma) as previously described ${ }^{14}$. Colonic mucins

368 oligosaccharides (cMO) were purified from pig distal pig colons and rectum. Briefly, the 369 tissue was open and the fecal contents were carefully removed. The mucosa was 370 scrapped off and mucus was extracted by homogenizing the tissue in at least 5 times 371 volume of extraction buffer (6 M guanidine chloride, $5 \mathrm{mM}$ EDTA, $10 \mathrm{mM} \mathrm{NaH} \mathrm{PO}_{4}$, $\mathrm{pH}$

3726.5 ) and slow stirring at $4^{\circ} \mathrm{C}$ for $16 \mathrm{~h}$. The solution was spun down at $15,000 \mathrm{rpm}$ and $37310^{\circ} \mathrm{C}$ for $30 \mathrm{~min}$ and supernatant was discharged. The pellets were resuspended in 
374 extraction buffer and the process was repeated until the supernatant was clear for at least 375 two extractions. After the extraction the mucins were solubilized by reducing the disulfide 376 bonds. The pellets were resuspended in fresh reduction buffer (6 M guanidine chloride, $3770.1 \mathrm{M}$ Tris, $5 \mathrm{mM}$ EDTA, pH 8.0) containing $25 \mathrm{mM}$ of 1,4-dithiothreitol and slowly stirred 378 at $37^{\circ} \mathrm{C}$ for $5 \mathrm{~h}$. After this incubation, $62.5 \mathrm{mM}$ of iodoacetamide were added and the 379 solution was stirred slowly in the dark at room temperature for $16 \mathrm{~h}$. The solution was 380 centrifuged at $10,000 \mathrm{rpm}$ at $4^{\circ} \mathrm{C}$ for $30 \mathrm{~min}$ and the supernatant containing the solubilized 381 mucins was extensively dialysed into water. Samples were dissolved into $100 \mathrm{mM}$ Tris$382 \mathrm{HCl} \mathrm{pH} 8.0$ containing $1 \mathrm{mg} / \mathrm{ml}$ of trypsin and incubated slowly stirring at $37^{\circ} \mathrm{C}$ for $16 \mathrm{~h}$.

383 The glycans were $\beta$-eliminated by adding $0.1 \mathrm{M} \mathrm{NaOH}$ and $1 \mathrm{M} \mathrm{NaBH}_{4}$ and incubate the 384 solution at $65^{\circ} \mathrm{C}$ for $18 \mathrm{~h}$. After cooling the solution to room temperature, the $\mathrm{pH}$ was 385 adjusted to 7.0 with concentrated $\mathrm{HCl}$ and extensively dialysed in water. The released 386 porcine colonic mucin glycans were recovered by lyophilization the solution until 387 completely dry and used in further experiments.

\section{HPLC and TLC sulfatase enzymatic assays}

390 The sulfatase activity screen against commercially available sulfated oligosaccharides 391 (Supplementary Table 2) was performed with $1 \mu \mathrm{M}$ of recombinant enzyme and $1 \mathrm{mM}$ 392 of substrate in $10 \mathrm{mM}$ MES pH6.5 with $5 \mathrm{mM} \mathrm{CaCl} 2$ for $16 \mathrm{~h}$ at $37^{\circ} \mathrm{C}$. Sulfated $\mathrm{N}$-acetyl-

393 D-lactosamine and lacto- $N$-biose were generated by incubating the respective sulfated 394 Lewis antigens with $1 \mu \mathrm{M}$ of $\alpha-1,3 / 1,4$-fucosidase BT1625 $5^{31}$ in the same conditions. 395 Reactions were analysed by thin layer chromatography (TLC). Briefly, $2 \mu \mathrm{L}$ of each 396 sample was spotted onto silica plates and resolved in butanol:acetic acid:water (2:1:1) 397 running buffer. The TLC plates were dried, and the sugars were visualized using 398 diphenylamine stain $(1 \mathrm{ml}$ of $37.5 \% \mathrm{HCl}, 2 \mathrm{ml}$ of aniline, $10 \mathrm{ml}$ of $85 \% \mathrm{H} 3 \mathrm{PO}, 100 \mathrm{ml}$ of 399 ethyl acetate and $2 \mathrm{~g}$ diphenylamine) and heated at $100^{\circ} \mathrm{C}$ for $20 \mathrm{~min}$. When relevant, the 400 enzymatic activity was confirmed by high-performance anionic exchange 401 chromatography (HPAEC) with pulsed amperometric detection using standard 402 methodology. The sugars (reaction substrate/products) were bound to a Dionex 403 CarboPac P100 column and eluted with an initial isocratic flow of $10 \mathrm{mM} \mathrm{NaOH}$ during 20 $404 \mathrm{~min}$ then a gradient of $10-100 \mathrm{mM}$ of $\mathrm{NaOH}$ for $20 \mathrm{~min}$ at a flow rate of $1.0 \mathrm{ml} \mathrm{min}^{-1}$. The 
reaction products were identified using the appropriated standards. All experiments were 406 performed in triplicate.

\section{Liquid Chromatograph-Electrospray Ionization Tandem Mass Spectrometry}

409 Enzymatic reactions of sulfatases in colonic mucin oligosaccharides and culture 410 supernatant were cleaned up with graphitized carbon ${ }^{32}$. Reactions with sulfated defined 411 saccharides were reduced and desalted. Briefly, reactions were dried in Speed vac, 412 reconstituted in $20 \mu \mathrm{L}$ of $50 \mathrm{mM} \mathrm{NaOH}$ and $500 \mathrm{mM} \mathrm{NaBH}_{4}$ and incubated at $50^{\circ} \mathrm{C}$ for 3 $413 \mathrm{~h}$. Reactions were cool down on ice, neutralized with $1 \mu \mathrm{L}$ of glacial acetic acid and 414 desalted using a cation exchange column containing $A G^{\circledR} 50 \mathrm{~W}-\mathrm{X} 8$ resin. All cleaned and 415 desalted reactions were reconstituted in water before analysis by liquid chromatograph416 electrospray ionization tandem mass spectrometry (LC-ESI/MS). The oligosaccharides 417 were separated on a column $(10 \mathrm{~cm} \times 250 \mu \mathrm{m})$ packed in-house with $5 \mu \mathrm{m}$ porous 418 graphite particles (Hypercarb, Thermo-Hypersil, Runcorn, UK). The oligosaccharides 419 were injected on to the column and eluted with a 0-40\% acetonitrile gradient in $10 \mathrm{mM}$ 420 ammonium bicarbonate over $46 \mathrm{~min}$ at a flow rate of $10 \mu \mathrm{l} / \mathrm{min}$.. A $40 \mathrm{~cm} \times 50 \mu \mathrm{m}$ i.d. 421 fused silica capillary was used as transfer line to the ion source. Samples were analyzed 422 in negative ion mode on a LTQ linear ion trap mass spectrometer (Thermo Electron, San 423 José, CA), with an lonMax standard ESI source equipped with a stainless steel needle 424 kept at $-3.5 \mathrm{kV}$. Compressed air was used as nebulizer gas. The heated capillary was 425 kept at $300^{\circ} \mathrm{C}$, and the capillary voltage was $-33 \mathrm{kV}$. Full scan $(\mathrm{m} / \mathrm{z} 380-2,000$, two 426 microscan, maximum $100 \mathrm{~ms}$, target value of 30,000) was performed, followed by data427 dependent $\mathrm{MS}^{2}$ scans (two microscans, maximum $100 \mathrm{~ms}$, target value of 10,000) with 428 normalized collision energy of $35 \%$, isolation window of 2.5 units, activation q=0.25 and 429 activation time $30 \mathrm{~ms}$ ). The threshold for $\mathrm{MS}^{2}$ was set to 300 counts. Data acquisition and 430 processing were conducted with Xcalibur software (Version 2.0.7). Glycans were 431 identified from their MS/MS spectra by manual annotation and validated by available 432 structures stored in Unicarb-DB database (2020-01 version) (Supplementary Fig. 1) ${ }^{33}$. 433 O-Glycan structural characterization was based on diagnostic fragment ions ${ }^{34}$.The 434 schematic glycosidic or cross-ring cleavages were assigned according to the Domon and 435 Costello nomenclacture ${ }^{35}$. For comparison of glycan abundance between samples, the 
436 individual glycan structures were quantified relative to the total content by integration of

437 the extracted ion chromatogram peak area using Progenesis QI. The area under the 438 curve (AUC) of each structure was normalized to the total AUC and expressed as a 439 percentage.

\section{Microfuidic-based enzymatic desulfation assays}

442 Sulfated carbohydrates were labelled at their reducing end with BODIPY which has a maximal emission absorbance of $\sim 503 \mathrm{~nm}$, which can be detected by the EZ Reader via LED-induced fluorescence. Non-radioactive mobility shift carbohydrate sulfation assays were optimised in solution with a 12-sipper chip coated with CR8 reagent using a PerkinEImer EZ Reader II system using EDTA-based separation buffer. This approach allows real-time kinetic evaluation of substrate de-sulfation ${ }^{36}$. Pressure and voltage settings were adjusted manually (1.8 psi, upstream voltage:2250 V, downstream voltage: $500 \mathrm{~V}$ ) to afford optimal separation of the sulfated product and unsulfated substrate with a sample (sip) time of $0.2 \mathrm{~s}$, and total assay times appropriate for the experiment. Individual de-sulfation assays were carried out at $28^{\circ} \mathrm{C}$ after assembly in a 384-well plate in a final volume of $80 \mu \mathrm{l}$ in the presence of substrate concentrations between 0.5 and 20 $\mu \mathrm{M}$ with $100 \mathrm{mM}$ Bis-Tris-Propane, $150 \mathrm{mM} \mathrm{NaCl}, 0.02 \%$ (v/v) Brij-35 and $5 \mathrm{mM} \mathrm{CaCl}_{2}$. The degree of de-sulfation was calculated by peak integration using EZ Reader software, which measures the sulfated carbohydrate:unsulfated carbohydrate ratio at each individua time-point. The activity of sulfatase enzymes was quantified in 'kinetic mode' by monitoring the amount of unsulfated glycan generated over the assay time, relative to control assay with no enzyme; with sulfate loss limited to $\sim 20 \%$ to prevent of substrate and to ensure assay linearity. $k_{c a t} / K_{\mathrm{M}}$ values, using the equation $\mathrm{V}_{0}=\left(\mathrm{V}_{\max } / K_{\mathrm{M}}\right) / \mathrm{S}$, were determined by linear regression analysis with GraphPad Prism software. Substrate concentrations were varied to ensure assay linearity, and substrate concentrations present were significantly $<K_{\mathrm{M}}$. 


\section{NMR desulfation assays}

467 NMR experiments, monitoring the de-sulfation of 6S-D-galactose and 6S- $N$-acetyl-D468 galactosamine, were conducted in $\mathrm{D}_{2} \mathrm{O}$ with $50 \mathrm{mM}$ sodium phosphate, $\mathrm{pH}$ 7.0, 469 supplemented with $150 \mathrm{mM} \mathrm{NaCl}$ at $25^{\circ} \mathrm{C}$ on a $800 \mathrm{MHz}$ Bruker Avance III spectrometer 470 equipped with a TCl CryoProbe and a $600 \mathrm{MHz}$ Bruker Avance II+ spectrometer, also 471 fitted with a TCl CryoProbe. 1D and 2D proton and TOCSY spectra (mixing time $80 \mathrm{~ms}$ ) 472 were measured using standard pulse sequences provided by the manufacturer. Spectra were processed and analysed using TopSpin 3.4A and TopSpin 4.0 software (Bruker).

474 Galactose integrals were recorded directly for the $\mathrm{C}(6) \mathrm{H}_{2}-\mathrm{OH}$ peak within the region 3.694 475 to $3.721 \mathrm{ppm}$, referenced to the combined C(2) peaks of D-galactose and 6S-D-galactose 476 with in the region 3.415 to $3.475 \mathrm{ppm}$. Similarly, 6S-N-acetyl-D-galactosamine integrals were recorded directly for the $\mathrm{C}(6) \mathrm{H}_{2}-\mathrm{OH}$ peak within the region 3.674 to $3.747 \mathrm{ppm}$, referenced to the combined C(4) peaks for $N$-acetyl-D-galactosamine and 6S- $N$-acetyl-Dgalactosamine in the region 3.925 to $3.968 p p m$.

480

\section{Differential scanning fluorimetry}

482 Thermal shift/stability assays (TSAs) were performed using a StepOnePlus Real-Time 483 PCR machine (LifeTechnologies) and SYPRO-Orange dye (emission maximum $570 \mathrm{~nm}$, 484 Invitrogen) as previously described ${ }^{37}$ with thermal ramping between 20 and $95^{\circ} \mathrm{C}$ in $0.3^{\circ} \mathrm{C}$ 485 step intervals per data point to induce denaturation in the presence or absence of various 486 carbohydrates as appropriate to the sulfatase being analysed. The melting temperature 487 (Tm) corresponding to the midpoint for the protein unfolding transition was calculated by 488 fitting the sigmoidal melt curve to the Boltzmann equation using GraphPad Prism, with $\mathrm{R}^{2}$ 489 values of $>0.99$. Data points after the fluorescence intensity maximum were excluded 490 from the fitting. Changes in the unfolding transition temperature compared with the control 491 curve $\left(\Delta T_{m}\right)$ were calculated for each ligand. A positive $\Delta T_{m}$ value indicates that the ligand 492 stabilises the protein from thermal denaturation, and confirms binding to the protein. All 493 TSA experiments were conducted using a final protein concentration of $5 \mu \mathrm{M}$ in $100 \mathrm{mM}$ 494 Bis-Tris-Propane (BTP), pH 7.0, and $150 \mathrm{mM} \mathrm{NaCl}$ supplemented with the appropriate ligand. When BT1622 3 S-Gal/GalNAc and BT1636 3 S-Gal were assessed against 3'S-LacNAc 
497 difference in the $T_{m}$ value of the proteins was observed. Three independent assays were 498 performed for each protein and protein ligand combination.

\section{Glycan labelling}

501 Sulfated saccharide samples were labelled according to a modification of the method 502 previously described reporting the formation of $\mathrm{N}$-glycosyl amines for 4,6-O-benzilidene 503 protected D-gluopyranose monosaccharides with aromatic amines ${ }^{38}$. Briefly, the 504 lyophilised sugar $(1 \mathrm{mg})$ was dissolved in $0.50 \mathrm{ml}$ anhydrous methanol in a $1.5 \mathrm{ml} \mathrm{screw}$ 505 top PTFE microcentrifuge tube. $0.1 \mathrm{mg}$, BODIPY-FL hydrazide (4,4-difluoro-5,7506 dimethyl-4-bora-3a,4a-diaza-s-indacene-3-propionic acid

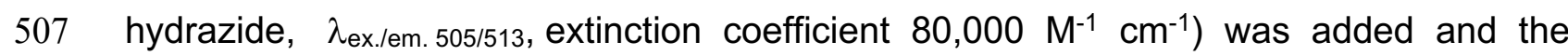
508 mixture vortexed $(1 \mathrm{~min})$, then incubated in darkness at $65^{\circ} \mathrm{C}$ for $24 \mathrm{~h}$. The products were 509 then cooled and a portion purified by TLC on silica coated aluminium plates and 510 developed with methanol or $1: 1 \mathrm{v} / \mathrm{v}$ ethyl acetate/methanol to provide $R_{\mathrm{f}}$ values suitable 511 to allow separation of unreacted label from labelled glycan product. The unreacted 512 BODIPY-FL label (orange on the TLC plate) was identified by reference to a lane 513 containing the starting material (BODIPY-FL hydrazide) on the TLC plate, allowing 514 differentiation from the putative labelled product (also orange). This latter band was 515 scraped from the plates and extracted in fresh methanol $(2 \times 0.5 \mathrm{ml})$, spun for $3 \mathrm{~min}$ at $51613,000 \times g$ and the supernatant was recovered and dried (rotary evaporator) to recover

517 the fluorescent-coloured product (bright green when dissolved in aqueous solution), 518 which was then employed in subsequent experiments. Anaerobic bacterial culture and genetic manipulation

521 All strains were anaerobic grown at $37{ }^{\circ} \mathrm{C}$ in a chamber $\left(10 \% \mathrm{H}_{2}, 5 \% \mathrm{CO}_{2}\right.$, and $85 \% \mathrm{~N}_{2}$; 522 Coy Manufacturing, Grass Lake, MI). Bacteroides type strains were culture in either 523 tryptone-yeast extract-glucose medium (TYG), brain heart infusion medium or minimal 524 medium (MM) containing an appropriate carbon source. Bacteroides massilliensis and 525 Akkermansia muciniphila were culture as described before ${ }^{21,39}$. Bt strains containing 526 specific gene deletions or inactivated versions of enzymes (BT1636 ${ }^{3 S-G a l}$ S77A) were 527 made by counterselectable allelic exchange as previously described ${ }^{40}$. Complemention 
528 of deletion strains was performed using pNBU2 vector as previously described ${ }^{40}$,

529 containing a constitutive promotor used previously ${ }^{41}$. All primers used to generate the 530 mutants and complementation are listed in Supplementary Table 9. Growth of the WT

531 and mutants was measured on an automated plate reader by increase in absorbance at

$532600 \mathrm{~nm}$ in 96-well plates containing $200 \mu \mathrm{l}$ of minimal media mixed with the respective

533 filter-sterilised (monosaccharide and $\mathrm{gMO}$ ) or autoclave-sterilised cMO as described

534 before $^{39}$. To achieve consistent growth, all carbon sources were used at $5 \mathrm{mg} / \mathrm{ml}$ with

535 exception of gMO that was added in a final concentration of $10 \mathrm{mg} / \mathrm{ml}$. All growth curves

536 presented are averages and s.e.m of three technical replicates.

\section{Crystallization of carbohydrate sulfatases}

539 After purification, all proteins were carried forward in the same eluent as used for the size

540 exclusion chromatography (see Recombinant Protein Production). Sparse matrix screens

541 were set up in 96-well sitting drop TTP Labtech plates (400-nL drops). Initial hits crystals

542 for all proteins were obtained between 20 and $35 \mathrm{mg} / \mathrm{ml}$. For BT1622 ${ }^{3 S-G a l / G a l N A c}$ and

543 BT1636 ${ }^{3 S-G a l}$ wildtype $B t$ variants were used, having a Ser at the catalytic formylglycine 544 position, whilst for for BT4683 35 -Gal the S73C mutant was used. BT1622 ${ }^{3 S-G a l / G a l N A c}$ with

$54520 \mathrm{mM}$ LNB crystallised in 20\% PEG 3350 and $0.2 \mathrm{M}$ sodium citrate tribasic dihydrate.

546 BT1636 ${ }^{3 \mathrm{~S}-G a l}$ with $20 \mathrm{mM}$ LacNAc crystallised in 40\% MPD and $0.2 \mathrm{M}$ sodium cacodylate 547 pH 6.5. for BT4683 ${ }^{3 S-G a l}$ with 20 mM LacNAc crystallised in 20\% PEG 3350, 0.2 M sodium 548 iodide and BTP pH 8.5. All crystals were cryoprotected with the addition of the ligand they 549 were crystallised with plus 20\% PEG 400 and 20\% glycerol was used as the 550 cryoprotectant for BT4683 3 S-Gal and BT1622 $3 \mathrm{~S}-$ Gal/GalNAc, respectively. No cryoprotectant 551 was added to BT1636 35 -Gal crystals. Data were collected at Diamond Light Source 552 (Oxford) on beamlines 103, 104, 104-1 and I24 at $100 \mathrm{~K}$. The data were integrated with $553 \mathrm{XDS}^{42}$, or Xia2 ${ }^{43} 3 \mathrm{di}$ or $3 \mathrm{dii}$ and scaled with Aimless ${ }^{44}$. Five percent of observations were 554 randomly selected for the $R_{\text {free }}$ set. The phase problem was solved by molecular 555 replacement using the automated molecular replacement server Balbes ${ }^{45}$ for all proteins 556 except BT1622 3 S-Gal/GalNAc. The phase problem for BT1622 ${ }^{3 S-G a l / G a l N A c}$ was initially solved 557 using Molrep ${ }^{46}$ and BT1636 ${ }^{3 S-G a l}$ as the search model. This gave a partial solution, which 558 could not be fully solved due to twinning. An acceptable model of BT1622 ${ }^{3 S-G a l / G a l N A c}$ was 
559 constructed to be used to better solve the phase problem and the molecular replacement 560 was re-performed. Models underwent recursive cycles of model building in $\operatorname{Coot}^{47}$ and 561 refinement cycles in Refmac5 ${ }^{48}$. Bespoke ligands were generated using JLigand ${ }^{49}$. The

562 models were validated using $\mathrm{Coot}^{47}$ and MolProbity ${ }^{50}$. Structural figures were made using 563 Pymol and all other programs used were from the CCP451 and CCP4i2 suite ${ }^{52}$. The data 564 processing and refinement statistics are reported in Supplementary Table 10.

\section{Immunolabelling of BT1636 in Bt cell surface}

For the fluorescence microscopy, Bt cells (Wild type ( $\Delta t d k)$ and $\Delta b t 1636^{3 S-G a l}$ ) were grown to early exponential phase (Abs600nm $0.25-0.35$ ) in rich TYG medium. One $\mathrm{ml}$ of the cultures was collected, centrifuged at 13,000 $\mathrm{g}$, and subsequently washed three times in MM with no carbon source. Bt cells incubated with cMO for four hours and fixed in $4.5 \%$ formalin overnight at $4^{\circ} \mathrm{C}$ with gentle rocking. Cells were stained with a polyclonal antibody raised in rabbit against purified recombinant BT1636 (BT1636 ${ }^{\mathrm{Ab}}$, Cocalico Biologicals) and detected with an Alexa Fluor ${ }^{\circledR}$ 488-conjugated goat anti-rabbit IgG secondary antibody (Molecular Probes). Images were taken with Zeiss Apotome using the same exposure time between samples.

\section{Gnotobiotic Mouse Experiments}

578 All experiments involving animals, including euthanasia via carbon dioxide asphyxiation,

579 were approved by the University Committee on Use and Care of Animals at the University

580 of Michigan (NIH Office of Laboratory Animal Welfare number A3114-01) and overseen

581 by a veterinarian. Groups of 3 to $5,6-8$ week old germfree Swiss Webster mice were 582 randomly assigned to each experiment. 7 days prior gavage the animals diet was 583 switched to a fiber-free diet (Envigo-Teklad TD 130343) that was maintained through all 584 the experiment. At day 0 , mice were gavage with equal amount of $B t$ WT strain and mutant 585 and fecal samples were collected at day 2 and every 5 days until day 42 . At the end-point 586 of the experiment distal small intestine and cecal contents were also collected. The 587 bacteria gDNA extraction and quantification by qPCR of the relative abundance of each 588 strain on the various samples was carried out as described previously ${ }^{39}$. 


\section{Phylogenetic analysis}

592 To maximise sequence coverage, and avoid repetition, we selected 800 and 920 593 representative sequences of subfamily S1_20 (composed of 1356 sequences) and S1_4 594 (composed of 1895 sequences), respectively. The sequences were aligned by MAFFT 595 v. $7^{53}$ using L-INS-i algorithm. The multiple sequence alignment was visualized by Jalview 596 software v.11.054 and non-aligned regions were removed. 404 and 364 positions were 597 used for the S1_4 and S1_20 phylogeny, respectively. Phylogeny was made using

598 RAxML v. 8.2.455. The phylogenetic tree was build with the Maximum Likelihood method ${ }^{56}$ 599 and the LG matrix as evolutive model ${ }^{57}$ using a discrete Gamma distribution to model 600 evolutionary rate differences among sites (4 categories). The rate variation model allowed 601 for some sites to be evolutionarily invariable. The reliability of the trees was tested by 602 bootstrap analysis using 1,000 resamplings of the dataset ${ }^{58}$. All the final global 603 phylogenetic trees were obtained with MEGA v. $7^{59}$. Fifteen S1_0 sequences from the 604 sulfAtlas database were used as an outgroup.

\section{Quantification and statistical analysis}

607 For in vivo competitions, when three or more fecal samples were collected, Student's t 608 tests (one-tailed, paired) were performed for each time point in Excel. When necessary, 609 the statistical analysis for remaining samples in stated in the respective figure legend.

\section{Data availability statement}

612 Source Data for all experiments, along with corresponding statistical test values, where 613 appropriate, are provided within the paper and in Supplementary information. The crystal 614 structure dataset generates have been deposit in the in the Protein Data Bank (PDB) 615 under the following accession numbers: 7ANB, 7ANA, 7AN1 and 7ALL. The LC-MS/MS 616 raw files and annotated structures are submitted to the Glycopost 617 (https://glycopost.glycosmos.org/preview/12430260615f9d5733a1a5d, code 1955) and 618 Unicarb-DB database, respectively.

620 Code availability statement: No new codes were developed or compiled in this study 


\section{Competing interests statement}

622 The authors declare no competing interests.

623

\section{Acknowledgements}

625 This project has received funding from the European Union's Horizon 2020 research and 626 innovation programme under the Marie Skłodowska-Curie grant agreement № 748336. 627 This work was supported by National Institutes of Health grants (DK118024 and 628 DK125445 awarded to ECM, U01AI095473 awarded to $\mathrm{GCH}$ ), the European Research 629 Council ERC (694181), The Knut and Alice Wallenberg Foundation (2017.0028), Swedish 630 Research Council (2017-00958) and the academy of medical sciences/Wellcome Trust 631 through the springboard grant SBF005\1065 163470 awarded to AC. The authors 632 acknowledge access to the SOLEIL and Diamond Light sources via both the University 633 of Liverpool and Newcastle university BAGs (proposals mx21970 and mx18598, 634 respectively). We thank the staff of DIAMOND, SOLEIL, and members of the Liverpool's 635 Molecular biophysics group for assistance with data collection. Mass spectrometry of 636 glycans was performed in the Swedish infrastructure for biologic mass spectrometry 637 (BioMS) supported by the Swedish Research Council. We are also grateful for Dr. Erwan 638 Corre's help regarding bioinformatics analyses (ABIMS platform, Station Biologique de 639 Roscoff, France).

\section{Author contributions}

642 ASL, AC and ECM designed experiments and wrote the manuscript. ASL and AC cloned, 643 generated proteins mutants, expressed and purified sulfatases. ASL and AC performed 644 enzyme assay, binding and kinetic analyses with assistance of DPB and PAE. MR and 645 SO synthesized the O3-sulfated N-acetylgalactosamine. EAY labelled glycan substrates. 646 AC grew, solved, collected and analysed crystallographic data with assistance from $A B$. 647 AC and JAL carried out NMR kinetic analyses. CJ, ASL, GCH and NGK performed and 648 interpreted analytical glycobiology experiments. ASL, GP, RWPG, SG, SS and NAP 649 performed bacterial growth experiments and analysed in vivo competition data. MC, GM 650 and TB performed sulfatase phylogenetic analyses. All authors read and approved the manuscript. 


\section{Figure legends}

654 Figure 1. Bacterial growth on colonic mucin and Bt sulfatase activities. a, 655 Schematic representation of mucin O-glycans and relevant terminal epitopes (dashed 656 boxes). Sugars are shown according to the Symbol Nomenclature for Glycan system ${ }^{60}$. 657 b, Growth of Bacteroides type strains and Akkermansia muciniphila on colonic mucin O658 glycans (cMO) and number of respective encoded S1 sulfatases. The bars represent the 659 average of two independent experiments with different batches of cMO. Bacterial species 660 able to utilize gastric mucin glycans are highlighted in blue. Maximum absorbance is the 661 difference of the maximum absorbance value (Abs600nm) for each culture and the initial 662 absorbance at time $0\left(\mathrm{~T}_{0 h}\right)$. Graphic shows the example of growth curves for $B$. fragilis $663(B f), B$. thetaiotaomicron $(B t), B$. vulgatus $(B v)$ and $B$. massilliensis $(B m)$. c, Phylogeny of 664 Bt sulfatases showing the 28 S1 sulfatases and their respective substrates where known, 665 including this study. Enzymes are color coded according the respective subfamilies with 666 sulfatases characterized in this study highlighted in bold. * indicates sulfatase activity 667 previously characterized and arrows point the substrate preferentially targeted by the 668 respective enzyme. Sulfatases on a shared branch that share more than $86 \%$ and 39 $66958 \%$ of sequence identity are highlighted in blue and green background, respectively. 670 Data from biological replicates $n=3$ to 6 and error bars denote s.e.m..

672 Figure 2. Activity of Bt sulfatases on colonic mucin O-glycans. a, Representation of 673 O-glycans detected by mass spectrometry in cMO (control) and after sulfatase treatment 674 from the lower (top) to the higher (bottom) mass range. b, Relative abundance and 675 putative structures for the specific $\mathrm{m} / \mathrm{z}$ shown in panel a. Remaining structures are shown 676 in Extended figure 5a. c, Schematic representation of the putative structures that were not detected after treatment with BT1636 3 S-Gal.

679 Figure 3. Crystal structures of 35-Gal/GalNAc sulfatases. a, Schematic 680 representation of the residues interacting with targeted sugars, including the putative 681 catalytic residues (in dark red), the calcium ion (grey sphere) and subsites S, 0 and +1 682 highlighted in red. BT1636 3 -Gal and BT46833S-Gal in complex with LacNAc (D-Gal- $\beta 1,4-$ 
683 D-GIcNAc) and BT16223s-Gal/GalNAc in complex with GalNAc. b, Surface representation of 684 the active pocket. The equivalent Gal/GalNAc specificity residues in BT1636 ${ }^{3 S-G a l}$ and 685 BT4683 ${ }^{3 S-G a l}$ are highlighted in red and blue. The open active site of BT46833S-Gal is 686 highlighted in purple. In all structures the amino acids and ligands are represented as 687 stick.

Figure 4. BT1636 ${ }^{3 S-G a l}$ activity is required for the utilization of $\mathrm{CMO}$ and competitive fitness in vivo. a, Growth of $B t$ wild-type $\Delta t d k(\mathrm{WT})$, different sulfatase gene-deletion mutants (named " $\Delta b t X X X X$ ") and strains complemented with bt1636"3s-Gal on colonic or gastric mucin O-glycans (cMO and gMO, respectively) (line represents the average of biological replicates $(n=3)$ and error bars denote s.e.m.) b, Immunofluorescent and differential interference contrast (DIC) microscopy of Bt WT and sulfatase mutant staining with polyclonal antibody (Ab) against BT1636 $36^{3 \mathrm{~S}-G a l}$ (green) and DNA staining with DAPI (blue). c, Relative abundance of different O-glycans detected by mass spectrometry in $\Delta b t 1636^{3 \mathrm{~S}-G a l}$ culture supernatant or $\mathrm{CMO}$ in minimal media without bacteria (control), after $96 \mathrm{~h}$ in anaerobic conditions. The mass and associated structure of the 3 more abundant glycans in both samples are shown. $\mathbf{d}$, in vivo competitions in gnotobiotic mice ( $n=5-9$ separate mice from two separate experiments, except competition of the $\triangle 6 S$-GIcNAC mutant that showed no defect in one experiment) fed fiber-free diet and inoculated with WT and mutants. The fecal relative abundance of each strain was determined along the time course and in small intestine and cecum at day 42 (experimental endpoint). The relative abundance in each mouse is represented in the respective light colour. The error bars denote s.e.m. Significant differences between wild-type and mutant strain were compared at each time point using student's t-test (paired, one tail) and * indicates sample days in which the mutant was significantly different $(p<0.01)$ from the wild-type. in different mucin O-glycans. The graphics show the growth of strains able to utilize 
$712 \mathrm{n}=3$, error bars denote the s.e.m.). cMO, colonic mucin O-glycans; gMO, gastric mucin

713 O-glycans.

715 Extended Data 2. Schematic representation of polysaccharide utilization loci

716 (PULs) encoding sulfatases (sulf). Genes are colour coded according to the predicted

717 function of the respective proteins. Glycoside hydrolases $(\mathrm{GH})$ in known families are

718 indicated by $\mathrm{GHXX}$ or $\mathrm{GH}^{*}$, where $\mathrm{XX}$ and * indicates the respective family number or

719 non-classified, respectively.

720

721 Extended Data 3. Enzymatic screen of Bt sulfatases using sulfated 722 monosaccharides. Recombinant enzymes $(1 \mu \mathrm{M})$ were incubated with $1 \mathrm{mM}$ of 723 substrate in $10 \mathrm{mM}$ MES pH6.5 with $5 \mathrm{mM} \mathrm{CaCl}_{2}$ for $16 \mathrm{~h}$ at $37^{\circ} \mathrm{C}$. Reactions were 724 analyzed by thin layer chromatography (left side) or HPAEC with pulsed amperometric 725 detection (right side). Control reactions without sulfatases were carried out in the same 726 conditions. The standards in TLC and HPAEC-PAD are labelled on the left side and top, 727 respectively. The different panel represent activities found for sulfatases targeting: (a) 4S728 Gal/GalNAc; (b) 6S-Gal/GalNAc; (c) 6S-GlcNAc and; (d) 3S-GlcNAc. The data shown 729 are a representative from biological replicates $(n=3)$.

730

731 Extended Data 4. Activity and affinity of sulfatases to targeted substrates. a,

732 Recombinant enzymes $(1 \mu \mathrm{M})$ were incubated with $1 \mathrm{mM}$ of substrate in $10 \mathrm{mM}$ MES $733 \mathrm{pH} 6.5$ with $5 \mathrm{mM} \mathrm{CaCl}_{2}$ for $16 \mathrm{~h}$ at $37^{\circ} \mathrm{C}$. Sulfated disaccharides were generated by 734 adding $1 \mu \mathrm{M}$ of a characterized $\alpha 1,3 / 1,4$-fucosidase (BT1625) in the enzymatic reaction.

735 Control reactions without sulfatases were carried in the same conditions. Samples were 736 analysed by mass spectrometry and the intensity of the substrate and reaction products 737 was used for comparison of the relative abundance of these sugars after incubation with 738 the respective enzymes. b, Affinity studies looking at the effect of ligand binding on the 739 melting temperature of $3 S$ and 6S-Gal sulfatases. All reactions were performed in 100 740 mM BTP, pH 7.0 with 150 mM NaCl. For sample melting temperatures see Table S11. c, 741 Activity of $3 S-G a l / G a l N A c$ sulfatases $(10 \mu \mathrm{M})$ against 3 S-GalNAc $(10 \mathrm{mM})$. Reactions 
742 were performed in $10 \mathrm{mM} \mathrm{Hepes,} \mathrm{pH} 7.0$, with $150 \mathrm{mM} \mathrm{NaCl}$ and $5 \mathrm{mM} \mathrm{CaCl}_{2}$. The data

743 shown are one representative from the biological replicates conducted $(n=3)$.

744

Extended Data 5. Activity of Bt sulfatases against colonic mucin O-glycans (cMO) analysed by mass spectrometry. a, Relative abundance of defined oligosaccharides after incubation of $\mathrm{cMO}$ with different sulfatases or no enzyme (control). The putative structure for the different mass is shown on the right side of the graphic. The reactions were performed with $1 \mu \mathrm{M}$ of enzyme and $0.5 \% \mathrm{cMO}$ in $10 \mathrm{mM}$ MES pH 6.5 with $5 \mathrm{mM}$ $\mathrm{CaCl}_{2}$ for $16 \mathrm{~h}$ at $37^{\circ} \mathrm{C}$. b, Relative abundance of structures detected in different samples organized by sulfate-linkage (top panel) or presence of one or several sugar substitutions such as sulfate, sialic acid and fucose (bottom panel). The colour-coded bars represent the relative abundance and the total number of the structures containing the specific linkage/substitution. The complete dataset is provided in Supplementary Table 4.

Extended Data 6. Schematic representation of 3S-Gal/GalNAc sulfatases. a, Cartoon representation colour ramped from blue $(\alpha / \beta / \alpha \mathrm{N}$-terminal domain) to red ( $\beta$-sheet $\mathrm{C}$ terminal domain). b, Overlay of the active site $S$ residues of BT1636 ${ }^{3 S-G a l}$ (green) BT1622 $2^{3 S-G a l / G a l N A c}$ (blue) and BT4683 ${ }^{3 S-G a l}$ (pink). The putative catalytic residues are shown in bold. The calcium ion is represented as a grey sphere and its polar interactions indicated as dashed lines. C, Ligand density of maps for LacNAc in BT1636 ${ }^{3 S-G a l}$ and BT4683 3 S-Gal , and GalNAc in BT1622 ${ }^{3 S-G a l / G a l N A c}$, contoured at $1 \sigma\left(0.33 \mathrm{e} / \mathrm{A}^{3}, 0.37 \mathrm{e} / \mathrm{A}^{3}\right.$ and $0.18 \mathrm{e} / \mathrm{A}^{3}$, respectively); $\mathbf{d}$, Docking of putative structures of $O$-glycans targeted by BT4683 3 S-Gal using the LacNAc as reference point showing that this structure can accommodate a sialic acid in -1 subsite and additional sugars in positive subsites (left hand side). The docking sugars are schematic shown as sticks (middle panel) and schematic represented inside the dashed box (right hand side). Using the LacNAc product as an 'anchor' additional sugars were built in manually with Coot 0.9 and regularized to low energy conformations.

Extended Data 7. Phylogenetic tree of S1_20 and S1_4 sulfatases. The radial trees were constructed using the branched trees shown in Supplementary Figs. 2 and 3. For 
773 clarity, all labels and sequence accession codes have been omitted. Red filled circles

774 designate sequences from $B$. thetaiotaomicron sulfatases. The residue is written in black

775 without any attributes if present in the sequence, in grey and italics if the residue is

776 mutated to any type in that sequence, or to a specific residue type if given in brackets. a,

777 Radial representation of the phylogenetic tree constructed with representative sequences

778 of the sulfatase S1_4 subfamily. The colour code is given as a pattern of presence or

779 absence of the residues R72, E335 and W505, which are crucial in substrate recognition

780 by BT4683 (acc-code Q89YP8, coloured red). A grey X in italics specifically designates

781 that the residue W505 is absent in that sequence, and no obvious orthologous residue

782 can be found from the alignment. $\mathbf{b}$, Radial representation of the phylogenetic tree

783 constructed with representative sequences of the sulfatase S1_20 subfamily. The colour

784 code is given as a pattern of presence or absence of the residues E100, Q173 H177,

785 E334, R353, which are crucial in substrate recognition by BT1636 (acc-code Q8A789,

786 coloured red). A grey $X$ in italics specifically designates that the residue E100 is absent

787 in that sequence, and no obvious ortologous residue can be found from the alignment.

789 Extended Data 8. Sulfatase activity is required for growth in cMO and in vivo

790 fitness. a, Growth curves of $B t$ wild-type $\Delta t d k(\mathrm{WT})$, different sulfatase mutants ( $\Delta b t X X X)$

791 and complemented strains on glucose, colonic or gastric mucin O-glycans (cMO and

$792 \mathrm{gMO}$, respectively). The curves represent the average of biological replicates $(n=3)$ and

793 the error bars denote s.e.m. b, Relative abundance of oligosaccharides detected by mass

794 spectrometry in culture supernatant of WT and $\Delta b t 1636^{3 S-G a l}$ after growth in cMO for $96 \mathrm{~h}$

795 at anaerobic conditions. The control corresponds to CMO incubated in the same 796 conditions without bacterium. The colours represent the relative abundance of structures

797 grouped according to the presence of epitopes (sulfate, fucose and sialic acid) and the 798 numbers represent the total number of structures that contain the respective substitution.

799 c, Colonization of gnotobiotic mice fed a fiber-free diet by Bt WT and mutants lacking the 800 full ( $\triangle a n S M E$, no S1 sulfatases active) or specific sulfatase activity ( $\triangle 6 S-G / c N A c$ and $801 \Delta 6 S-G / c N A c+\Delta 6 S-G a l / G a l N A c)$. The fecal relative abundance of each strain was 802 determined in regular intervals until day 42. The relative abundance of time 0 represents 803 the abundance in gavaged inoculum. At the experimental endpoint the relative abundance 
was also determined in small intestine and cecum. The graphics represent the average of $n=3$ and the error bars denote the s.e.m. The relative abundance in each individual animal is represented in a lighter colour in each of the respective graphics.

\section{References}

1 Packey, C. D. \& Sartor, R. B. Commensal bacteria, traditional and opportunistic pathogens, dysbiosis and bacterial killing in inflammatory bowel diseases.

Current opinion in infectious diseases 22, 292-301 (2009)

2 Sears, C. L. \& Garrett, W. S. Microbes, Microbiota, and Colon Cancer. Cell Host Microbe 15, 317-328, doi:10.1016/j.chom.2014.02.007 (2014).

3 Johansson, M. E. et al. The inner of the two Muc2 mucin-dependent mucus layers in colon is devoid of bacteria. Proceedings of the National Academy of Sciences of the United States of America 105, 15064-15069, doi:10.1073/pnas.0803124105 (2008).

4 Van der Sluis, M. et al. Muc2-deficient mice spontaneously develop colitis, indicating that Muc2 is critical for colonic protection. Gastroenterology 131, 117129, doi:Doi 10.1053/J.Gastro.2006.04.020 (2006).

5 Velcich, A. et al. Colorectal cancer in mice genetically deficient in the mucin Muc2. Science 295, 1726-1729, doi:10.1126/science.1069094 (2002).

6 Bergstrom, K. et al. Core 1- and 3-derived O-glycans collectively maintain the colonic mucus barrier and protect against spontaneous colitis in mice. Mucosal immunology, doi:10.1038/mi.2016.45 (2016).

7 Larsson, J. M. et al. Altered O-glycosylation profile of MUC2 mucin occurs in active ulcerative colitis and is associated with increased inflammation. Inflamm Bowel Dis 17, 2299-2307, doi:10.1002/ibd.21625 (2011).

$8 \mathrm{Fu}, \mathrm{J}$. et al. Loss of intestinal core 1-derived O-glycans causes spontaneous colitis in mice. J Clin Invest 121, 1657-1666, doi:10.1172/JCI45538 (2011).

9 Kudelka, M. R. et al. Cosmc is an X-linked inflammatory bowel disease risk gene that spatially regulates gut microbiota and contributes to sex-specific risk. Proceedings of the National Academy of Sciences of the United States of America 113, 14787-14792, doi:10.1073/pnas.1612158114 (2016).

10 Larsson, J. M., Karlsson, H., Sjovall, H. \& Hansson, G. C. A complex, but uniform O-glycosylation of the human MUC2 mucin from colonic biopsies analyzed by nanoLC/MSn. Glycobiology 19, 756-766, doi:10.1093/glycob/cwp048 (2009).

11 Holmen Larsson, J. M., Thomsson, K. A., Rodriguez-Pineiro, A. M., Karlsson, H. \& Hansson, G. C. Studies of mucus in mouse stomach, small intestine, and colon. III. Gastrointestinal Muc5ac and Muc2 mucin O-glycan patterns reveal a regiospecific distribution. American journal of physiology. Gastrointestinal and liver physiology 305, G357-363, doi:10.1152/ajpgi.00048.2013 (2013).

12 Thomsson, K. A. et al. Detailed O-glycomics of the Muc2 mucin from colon of wild-type, core 1- and core 3-transferase-deficient mice highlights differences 
compared with human MUC2. Glycobiology 22, 1128-1139, doi:10.1093/glycob/cws083 (2012).

13 Robbe, C. et al. Evidence of regio-specific glycosylation in human intestinal mucins - Presence of an acidic gradient along the intestinal tract. Journal of Biological Chemistry 278, 46337-46348, doi:DOI 10.1074/jbc.M302529200 (2003).

14 Martens, E. C., Chiang, H. C. \& Gordon, J. I. Mucosal Glycan Foraging Enhances Fitness and Transmission of a Saccharolytic Human Gut Bacterial Symbiont. Cell Host Microbe 4, 447-457, doi:DOI 10.1016/j.chom.2008.09.007 (2008).

15 Benjdia, A., Martens, E. C., Gordon, J. I. \& Berteau, O. Sulfatases and a radical S-adenosyl-L-methionine (AdoMet) enzyme are key for mucosal foraging and fitness of the prominent human gut symbiont, Bacteroides thetaiotaomicron. $J$ Biol Chem 286, 25973-25982, doi:10.1074/jbc.M111.228841 (2011).

16 Hickey, C. A. et al. Colitogenic Bacteroides thetaiotaomicron Antigens Access Host Immune Cells in a Sulfatase-Dependent Manner via Outer Membrane Vesicles. Cell Host Microbe 17, 672-680, doi:10.1016/j.chom.2015.04.002 (2015).

17 Diez-Roux, G. \& Ballabio, A. Sulfatases and human disease. Annu Rev Genomics Hum Genet 6, 355-379, doi:10.1146/annurev.genom.6.080604.162334 (2005).

18 Wlodarska, M. et al. Indoleacrylic Acid Produced by Commensal Peptostreptococcus Species Suppresses Inflammation. Cell Host Microbe 22, 25-37 e26, doi:10.1016/j.chom.2017.06.007 (2017).

19 Tramontano, M. et al. Nutritional preferences of human gut bacteria reveal their metabolic idiosyncrasies. Nat Microbio/ 3, 514-522, doi:10.1038/s41564-0180123-9 (2018).

20 Derrien, M., Vaughan, E. E., Plugge, C. M. \& de Vos, W. M. Akkermansia muciniphila gen. nov., sp. nov., a human intestinal mucin-degrading bacterium. Int J Syst Evol Microbiol 54, 1469-1476 (2004).

21 Pudlo, N. A. et al. Symbiotic Human Gut Bacteria with Variable Metabolic Priorities for Host Mucosal Glycans. mBio 6, e01282-01215, doi:10.1128/mBio.01282-15 (2015).

22 Barbeyron, T. et al. Matching the Diversity of Sulfated Biomolecules: Creation of a Classification Database for Sulfatases Reflecting Their Substrate Specificity. PLoS ONE 11, e0164846, doi:10.1371/journal.pone.0164846 (2016).

23 Benjdia, A. et al. Anaerobic sulfatase-maturating enzymes, first dual substrate radical S-adenosylmethionine enzymes. J Biol Chem 283, 17815-17826, doi:10.1074/jbc.M710074200 (2008).

24 Cartmell, A. et al. How members of the human gut microbiota overcome the sulfation problem posed by glycosaminoglycans. Proceedings of the National Academy of Sciences of the United States of America 114, 7037-7042, doi:10.1073/pnas.1704367114 (2017).

25 Ndeh, D. et al. Metabolism of multiple glycosaminoglycans by Bacteroides thetaiotaomicron is orchestrated by a versatile core genetic locus. Nature communications 11, 646, doi:10.1038/s41467-020-14509-4 (2020). 
26 Thomsson, K. A., Karlsson, H. \& Hansson, G. C. Sequencing of sulfated oligosaccharides from mucins by liquid chromatography and electrospray ionization tandem mass spectrometry. Analytical chemistry 72, 4543-4549, doi:10.1021/ac000631b (2000).

27 Thomsson, K. A., Backstrom, M., Holmen Larsson, J. M., Hansson, G. C. \& Karlsson, $\mathrm{H}$. Enhanced detection of sialylated and sulfated glycans with negative ion mode nanoliquid chromatography/mass spectrometry at high $\mathrm{pH}$. Analytical chemistry 82, 1470-1477, doi:10.1021/ac902602e (2010).

28 van der Post, S. \& Hansson, G. C. Membrane protein profiling of human colon reveals distinct regional differences. Mol Cell Proteomics 13, 2277-2287, doi:10.1074/mcp.M114.040204 (2014).

29 Tobisawa, Y., Imai, Y., Fukuda, M. \& Kawashima, H. Sulfation of colonic mucins by $\mathrm{N}$-acetylglucosamine 6 -O-sulfotransferase-2 and its protective function in experimental colitis in mice. J Biol Chem 285, 6750-6760, doi:10.1074/jbc.M109.067082 (2010).

30 Egan, M., Jiang, H., O'Connell Motherway, M., Oscarson, S. \& van Sinderen, D. Glycosulfatase-Encoding Gene Cluster in Bifidobacterium breve UCC2003. Appl Environ Microbiol 82, 6611-6623, doi:10.1128/AEM.02022-16 (2016).

31 Briliute, J. et al. Complex N-glycan breakdown by gut Bacteroides involves an extensive enzymatic apparatus encoded by multiple co-regulated genetic loci. Nat Microbiol 4, 1571-1581, doi:10.1038/s41564-019-0466-x (2019).

32 Packer, N. H., Lawson, M. A., Jardine, D. R. \& Redmond, J. W. A general approach to desalting oligosaccharides released from glycoproteins. Glycoconjugate journal 15, 737-747, doi:10.1023/a:1006983125913 (1998).

33 Hayes, C. A. et al. UniCarb-DB: a database resource for glycomic discovery. Bioinformatics 27, 1343-1344, doi:10.1093/bioinformatics/btr137 (2011).

34 Everest-Dass, A. V., Abrahams, J. L., Kolarich, D., Packer, N. H. \& Campbell, M. $\mathrm{P}$. Structural feature ions for distinguishing $\mathrm{N}$ - and $\mathrm{O}$-linked glycan isomers by LC-ESI-IT MS/MS. J Am Soc Mass Spectrom 24, 895-906, doi:10.1007/s13361013-0610-4 (2013).

35 Domon, B. \& Costello, C. E. Structure elucidation of glycosphingolipids and gangliosides using high-performance tandem mass spectrometry. Biochemistry 27, 1534-1543, doi:10.1021/bi00405a021 (1988).

36 Byrne, D. P. et al. New tools for carbohydrate sulfation analysis: heparan sulfate 2-O-sulfotransferase (HS2ST) is a target for small-molecule protein kinase inhibitors. Biochem J 475, 2417-2433, doi:10.1042/BCJ20180265 (2018).

37 Byrne, D. P. et al. cAMP-dependent protein kinase (PKA) complexes probed by complementary differential scanning fluorimetry and ion mobility-mass spectrometry. Biochem J 473, 3159-3175, doi:10.1042/BCJ20160648 (2016). 38 Das, T. M., Rao, C. P. \& Kolehmainen, E. Synthesis and characterisation of Nglycosyl amines from the reaction between 4,6-O-benzylidene-D-glucopyranose and substituted aromatic amines and also between 2-(oaminophenyl)benzimidazole and pentoses or hexoses. Carbohydrate research 334, 261-269, doi:10.1016/s0008-6215(01)00202-6 (2001). 
39 Desai, M. S. et al. A Dietary Fiber-Deprived Gut Microbiota Degrades the Colonic Mucus Barrier and Enhances Pathogen Susceptibility. Cell 167, 1339-1353 e1321, doi:10.1016/j.cell.2016.10.043 (2016).

40 Koropatkin, N. M., Martens, E. C., Gordon, J. I. \& Smith, T. J. Starch catabolism by a prominent human gut symbiont is directed by the recognition of amylose helices. Structure 16, 1105-1115, doi:10.1016/j.str.2008.03.017 (2008).

41 Degnan, P. H., Barry, N. A., Mok, K. C., Taga, M. E. \& Goodman, A. L. Human gut microbes use multiple transporters to distinguish vitamin $\mathrm{B}(1)(2)$ analogs and compete in the gut. Cell Host Microbe 15, 47-57, doi:10.1016/j.chom.2013.12.007 (2014).

42 Kabsch, W. Xds. Acta Crystallogr D Biol Crystallogr 66, 125-132, doi:10.1107/S0907444909047337 (2010).

43 Winter, S. E., Lopez, C. A. \& Baumler, A. J. The dynamics of gut-associated microbial communities during inflammation. EMBO Rep 14, 319-327, doi:10.1038/embor.2013.27 (2013).

44 Evans, P. R. \& Murshudov, G. N. How good are my data and what is the resolution? Acta Crystallogr D Biol Crystallogr 69, 1204-1214, doi:10.1107/S0907444913000061 (2013).

45 Long, F., Vagin, A. A., Young, P. \& Murshudov, G. N. BALBES: a molecularreplacement pipeline. Acta Crystallogr D Biol Crystallogr 64, 125-132, doi:10.1107/S0907444907050172 (2008).

46 Vagin, A. \& Teplyakov, A. Molecular replacement with MOLREP. Acta Crystallogr D Biol Crystallogr 66, 22-25, doi:10.1107/S0907444909042589 (2010).

47 Emsley, P., Lohkamp, B., Scott, W. G. \& Cowtan, K. Features and development of Coot. Acta Crystallogr D Biol Crystallogr 66, 486-501, doi:10.1107/S0907444910007493 (2010).

48 Murshudov, G. N. et al. REFMAC5 for the refinement of macromolecular crystal structures. Acta Crystallogr D Biol Crystallogr 67, 355-367, doi:10.1107/S0907444911001314 (2011).

49 Lebedev, A. A. et al. JLigand: a graphical tool for the CCP4 template-restraint library. Acta Crystallogr D Biol Crystallogr 68, 431-440, doi:10.1107/S090744491200251X (2012).

50 Chen, V. B. et al. MolProbity: all-atom structure validation for macromolecular crystallography. Acta Crystallogr D Biol Crystallogr 66, 12-21, doi:10.1107/S0907444909042073 (2010).

51 Collaborative computational project, $\mathrm{n}$. The CCP4 suite: Programs for protein crystallography. Acta Crystallographica D50, 760-763 (1994).

52 Potterton, L. et al. CCP4i2: the new graphical user interface to the CCP4 program suite. Acta Crystallogr D Struct Biol 74, 68-84, doi:10.1107/S2059798317016035 (2018).

53 Katoh, K., Misawa, K., Kuma, K. \& Miyata, T. MAFFT: a novel method for rapid multiple sequence alignment based on fast Fourier transform. Nucleic Acids Res 30, 3059-3066, doi:10.1093/nar/gkf436 (2002).

54 Clamp, M., Cuff, J., Searle, S. M. \& Barton, G. J. The Jalview Java alignment editor. Bioinformatics 20, 426-427, doi:10.1093/bioinformatics/btg430 (2004). 
98255 Stamatakis, A. RAxML version 8: a tool for phylogenetic analysis and postanalysis of large phylogenies. Bioinformatics 30, 1312-1313, doi:10.1093/bioinformatics/btu033 (2014).

56 Felsenstein, J. Evolutionary trees from DNA sequences: a maximum likelihood approach. Journal of molecular evolution 17, 368-376, doi:10.1007/BF01734359 (1981).

57 Le, S. Q. \& Gascuel, O. An improved general amino acid replacement matrix. Mol Biol Evol 25, 1307-1320, doi:10.1093/molbev/msn067 (2008).

58 Felsenstein, J. Confidence Limits on Phylogenies: An Approach Using the Bootstrap. Evolution 39, 783-791, doi:10.1111/j.1558-5646.1985.tb00420.x (1985).

59 Kumar, S., Stecher, G. \& Tamura, K. MEGA7: Molecular Evolutionary Genetics Analysis Version 7.0 for Bigger Datasets. Mol Biol Evol 33, 1870-1874, doi:10.1093/molbev/msw054 (2016).

60 Neelamegham, S. et al. Updates to the Symbol Nomenclature for Glycans guidelines. Glycobiology 29, 620-624, doi:10.1093/glycob/cwz045 (2019). 


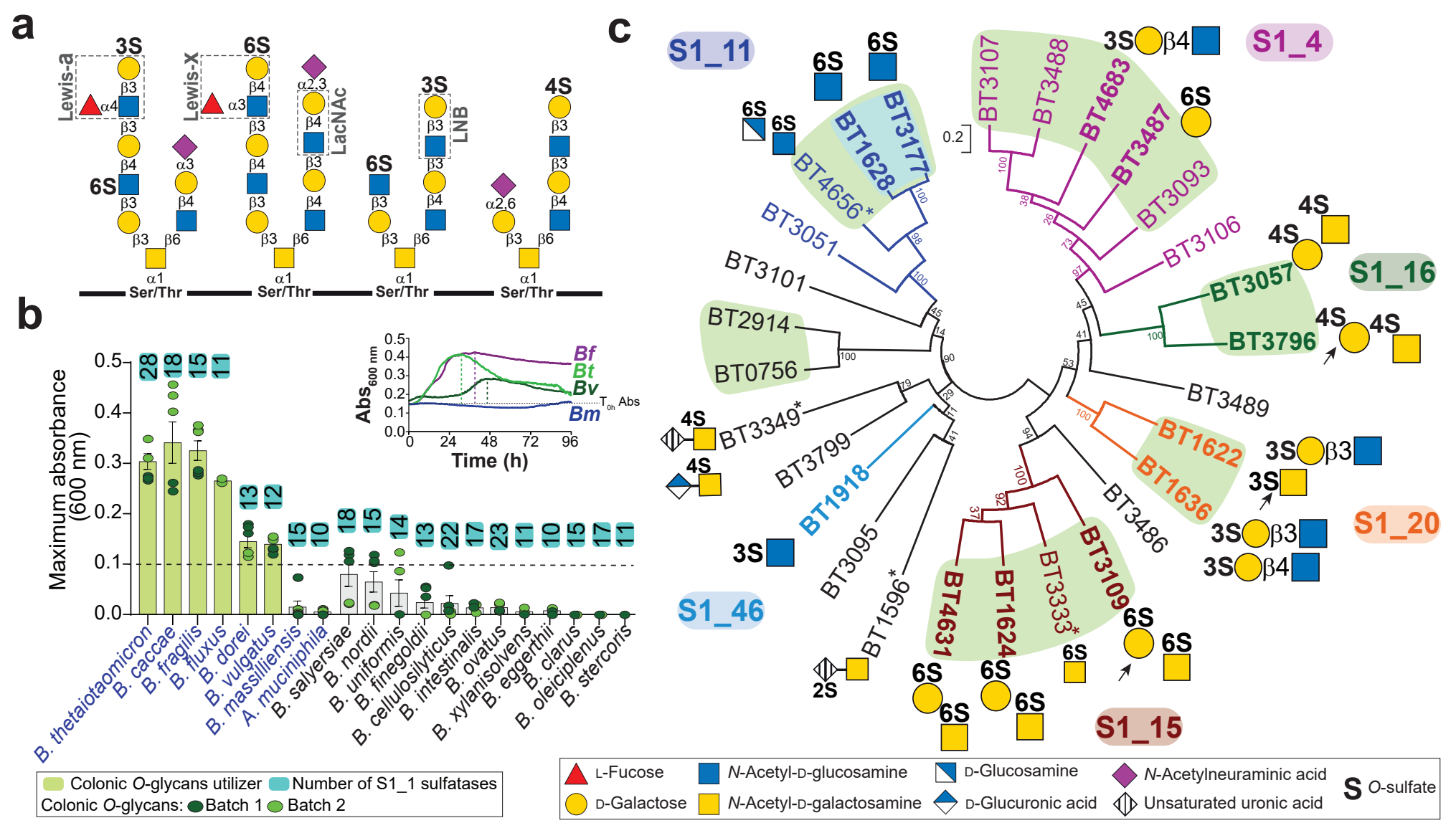

Fig 1. 
a
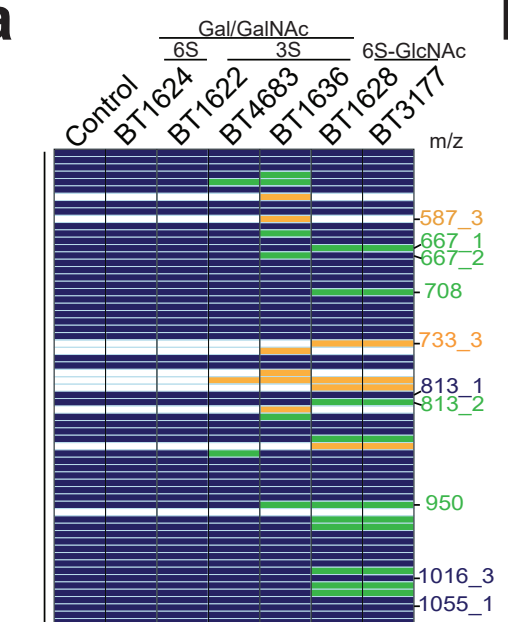

है
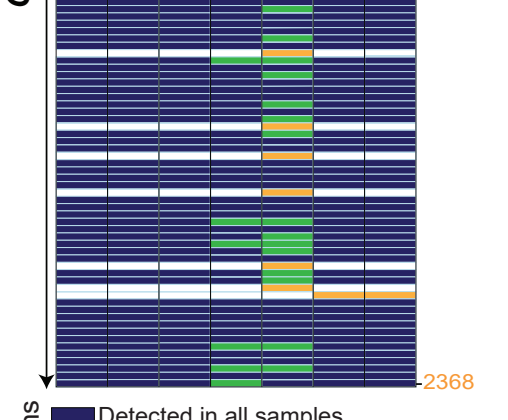

(2) Detected in all samples

ర్ $\square$ Not detect after enzyme incubation

ô $\square$ Only detected after enzyme incubation

○े $\square$ Not detected b
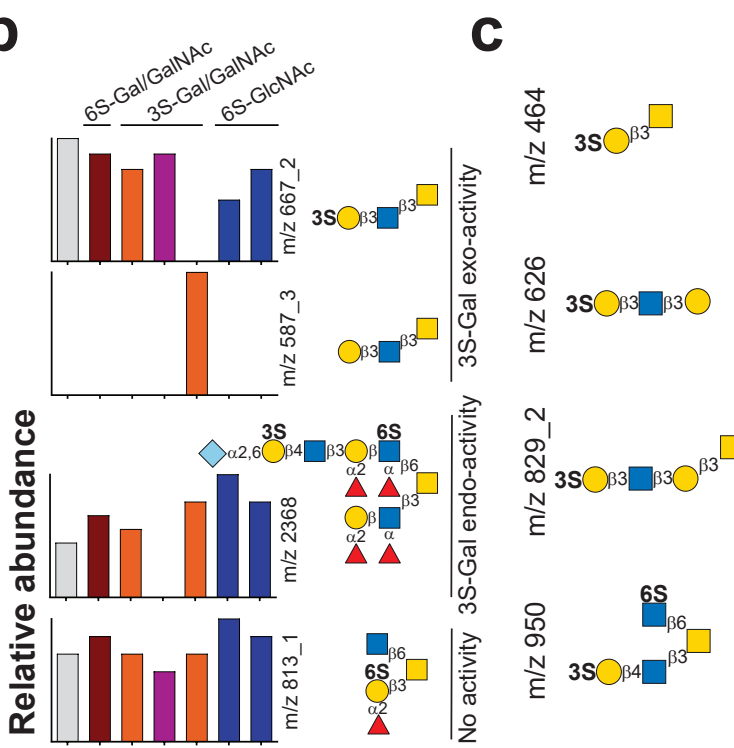

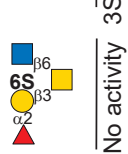

용 $\quad \mathrm{SS}^{66}$

$\stackrel{N}{\varepsilon} 3 \mathrm{~S} \bigcirc \beta 4 \square^{\beta 3}$

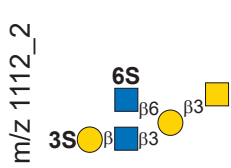

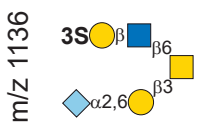

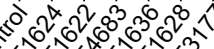
0 की

$S$ O-sulfate $\triangle$ L-Fucose ${ }_{D}$-Galactose

N-Acetyl-D-glucosamine

N-Acetyl-D-galactosamine

$\mathrm{N}$-Glycolylneuraminic acid

Sulfatase subfamily: 4

S1_11

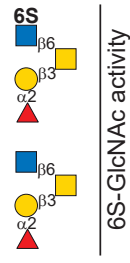

$\underset{N}{N} 35 \bigcirc \beta \square \square 33 \bigcirc^{\beta 3}$

है

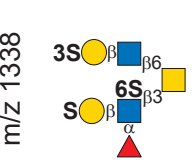



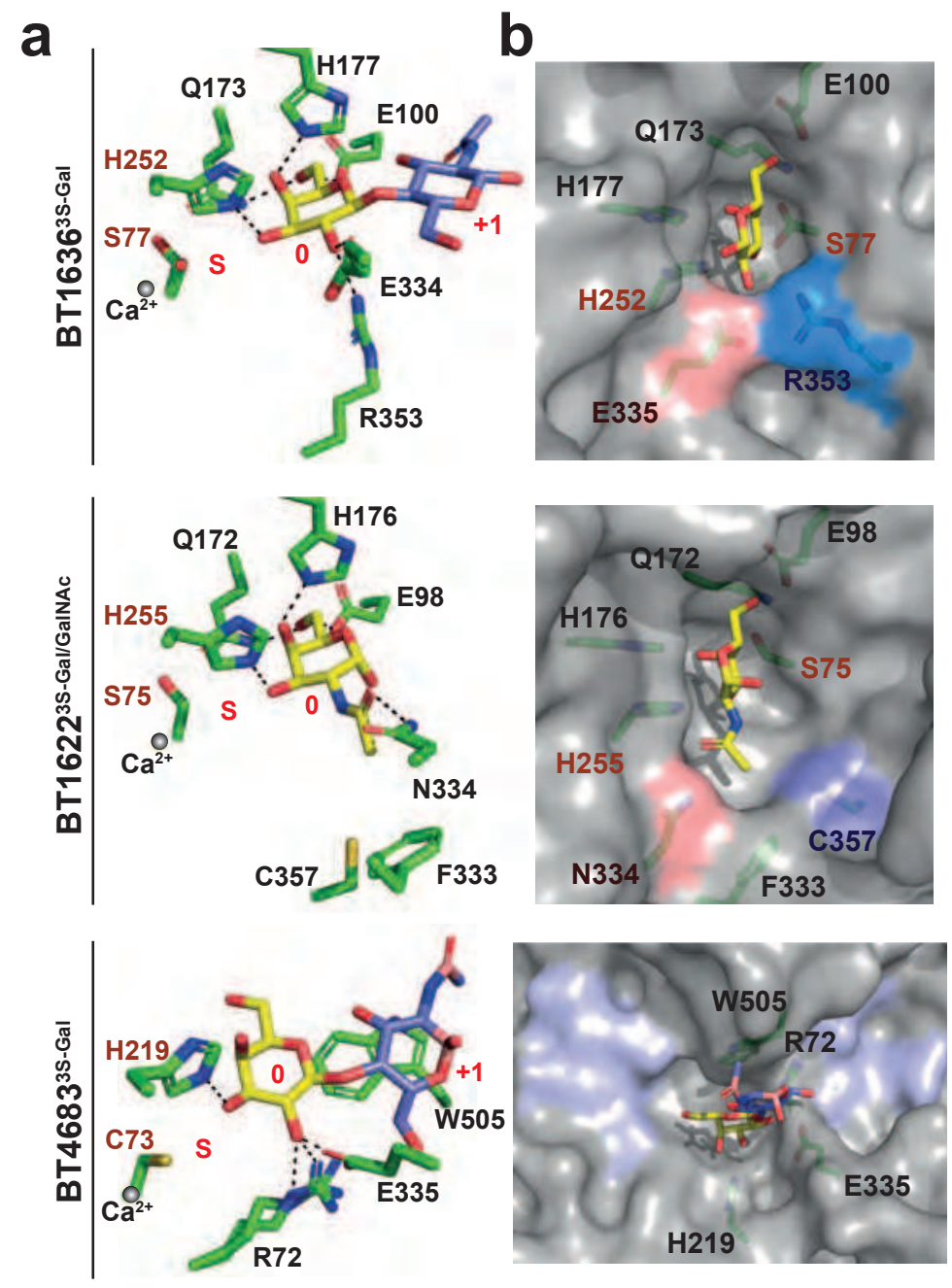

Fig 3. 
a

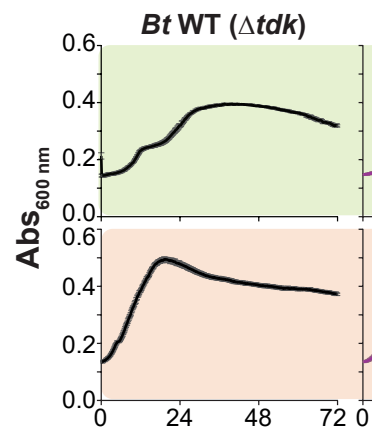

$\triangle$ anSME

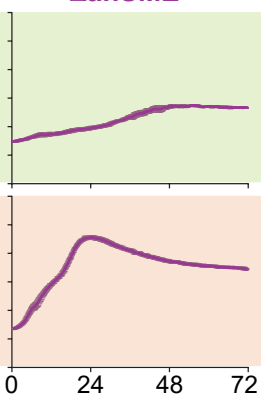

$\Delta b t 1636:: b t 1636^{3 s-G a l}$

$\Delta b t 1636^{3 s-G a l}$

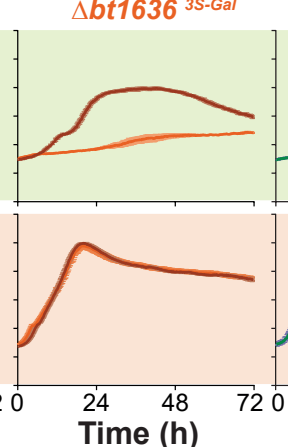

$\Delta 8 x$ sulf

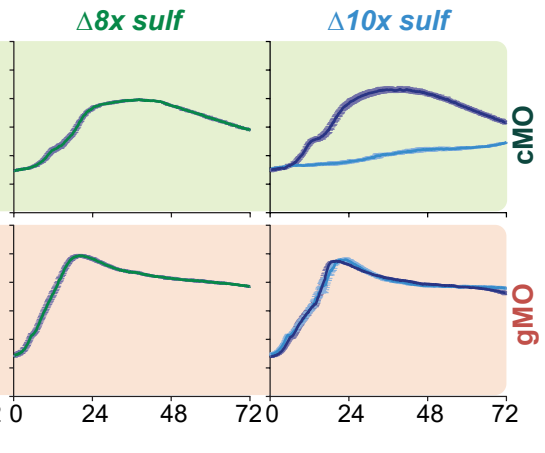

$\Delta 8 \mathrm{x}$ sulf $(\underline{\Delta b t 1622+\Delta b t 4683}+\underline{\Delta b t 1624+\Delta b t 3109+\Delta b t 4631}+\underline{\Delta b t 1628+\Delta b t 3177+\Delta b t 3051)}$ $\Delta 10 x$ sulf $\left[\frac{\text { bSt1636(S77A })+\Delta b t 1622+\Delta b t 4683}{\Delta b t 1624+\Delta b t 3109+\Delta b t 4631}+\frac{6 S-G l c N A c}{\Delta b t 1628+\Delta b t 3177}+\frac{\text { 4S-Gal }}{\Delta b t 3057+\Delta b t 3796}\right]$

C

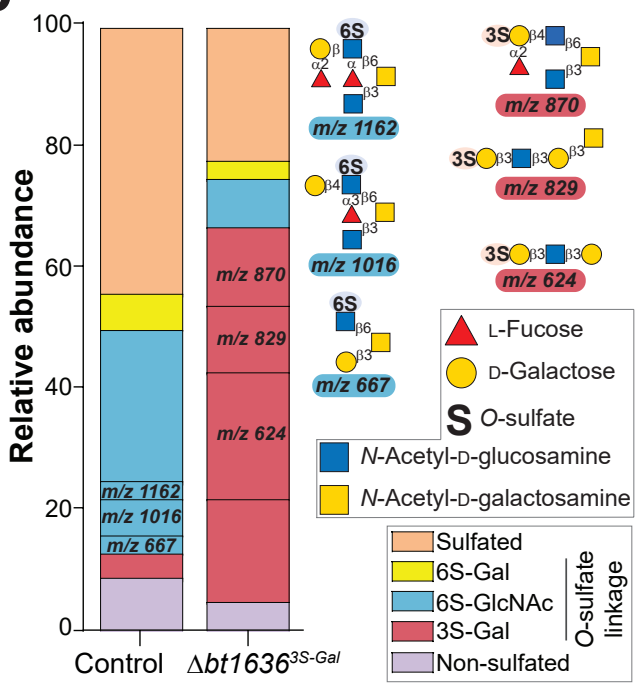

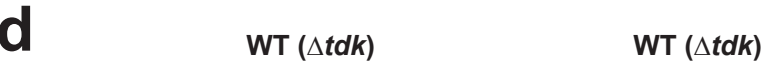

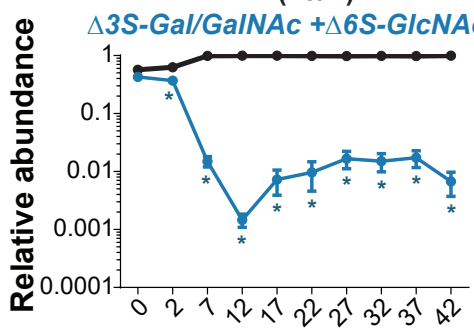

$\triangle$ SS-Gal/GalNAC

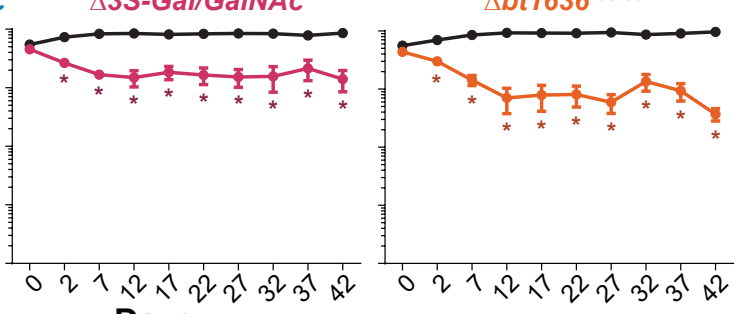

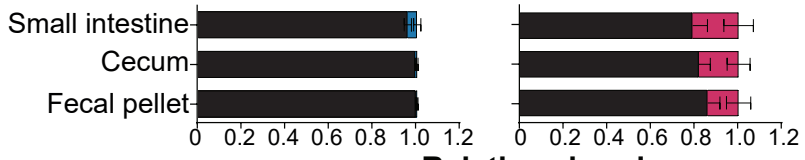

Relative abundance

$\Delta 3 S-G a l / G a l N A c+\Delta 6 S-G I c N A c\left[\Delta b t 1636(S 77 A)+\Delta b t 1622+\Delta b t 4683+\frac{\Delta b t 1628+\Delta b t 3177+\Delta b t 3051]}{6 S-G l c N A c}\right.$ $\Delta 3 S-G a l / G a l N A c[\Delta b t 1636(S 77 A)+\Delta b t 1622+\Delta b t 4683]$

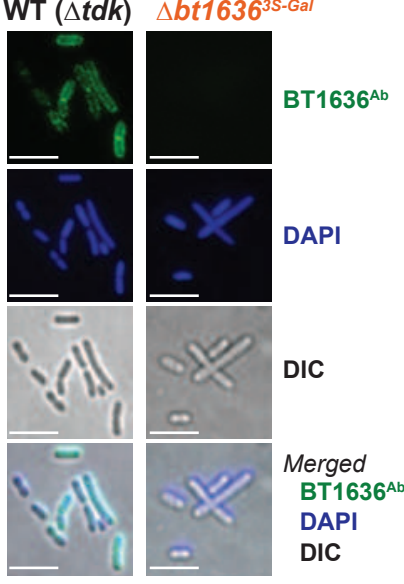




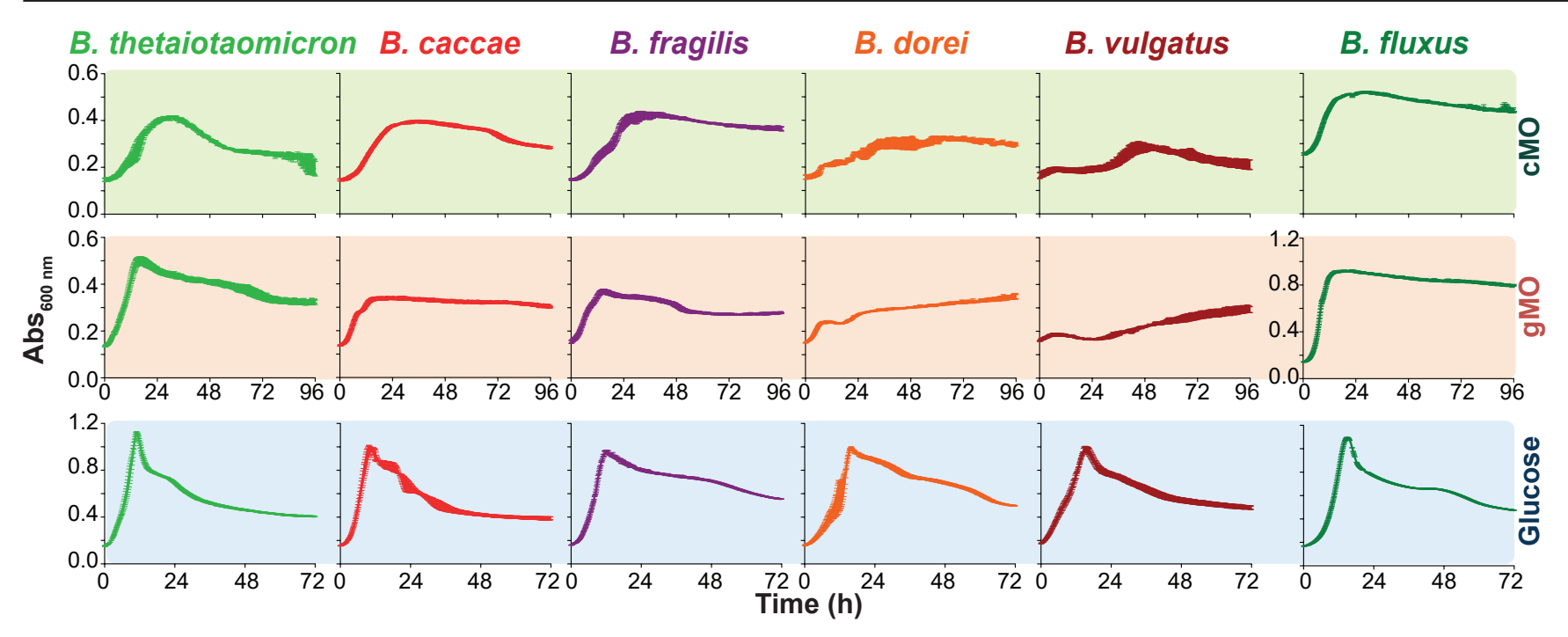

\section{Gastric O-glycans utilizer}

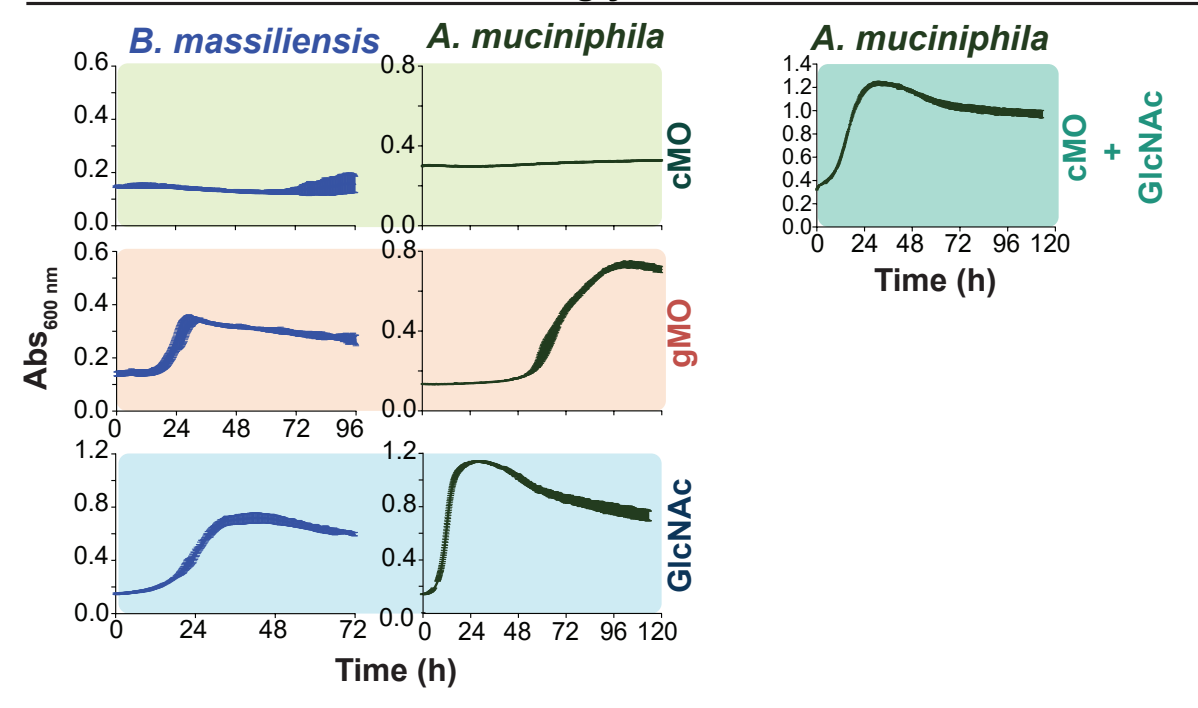



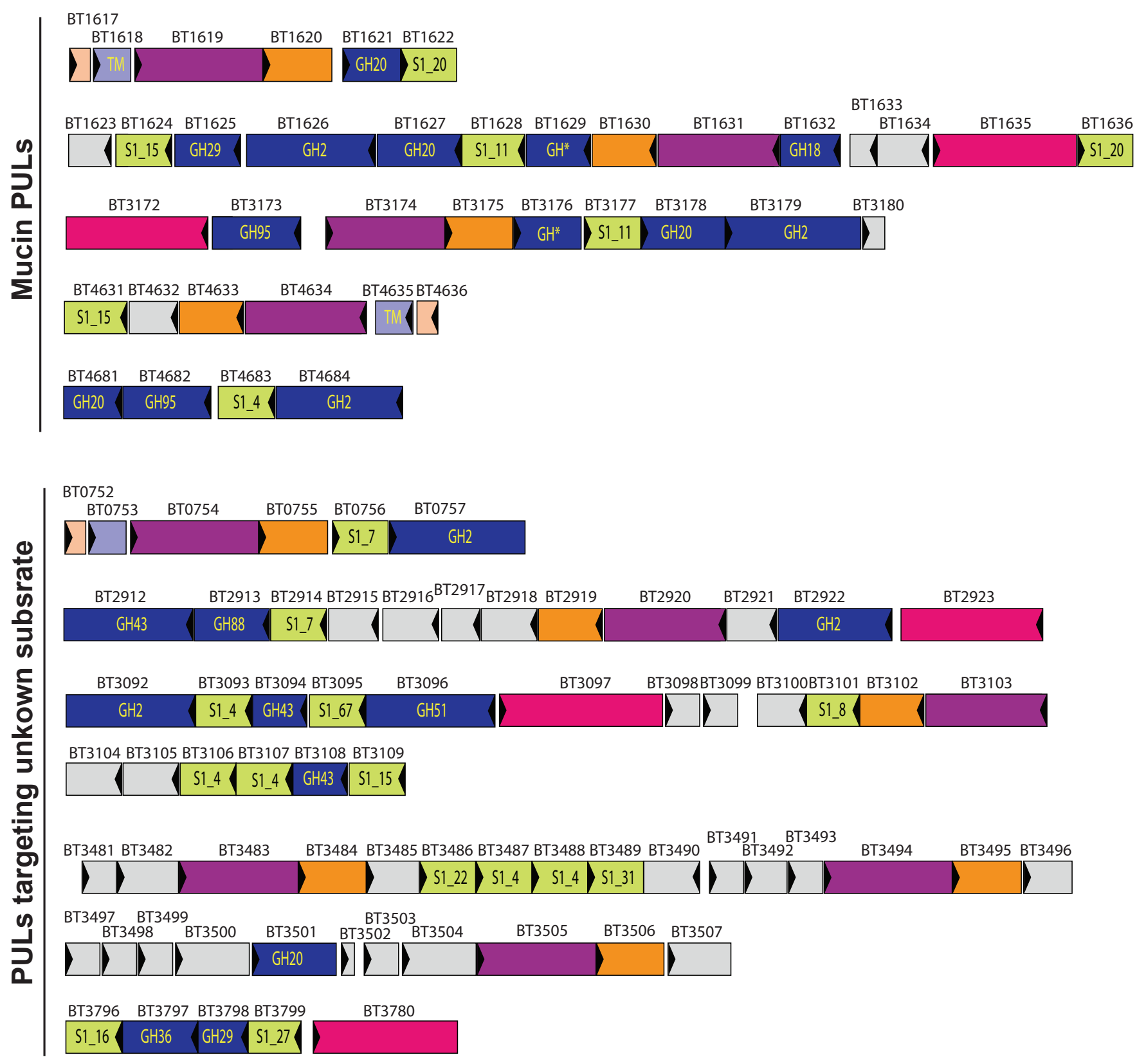

\begin{tabular}{|c|c|c|}
\hline$\square$ GH (Glycoside hydrolase) & $\square$ SusC-like & $\square$ Extra-cytoplasmic function sigma (ECF- $\sigma$ ) factor \\
\hline$\square$ S1 (Sulfatase) & $\square$ SusD-Like & $\square$ HTCS (Hybrid two component sytem) \\
\hline$\square$ HP (Hypothetical Protein) & $\square$ Anti-sigma (anti- $\sigma$ ) factor & $\square$ TM (Transcriptional regulator with transmembrane domain) \\
\hline
\end{tabular}



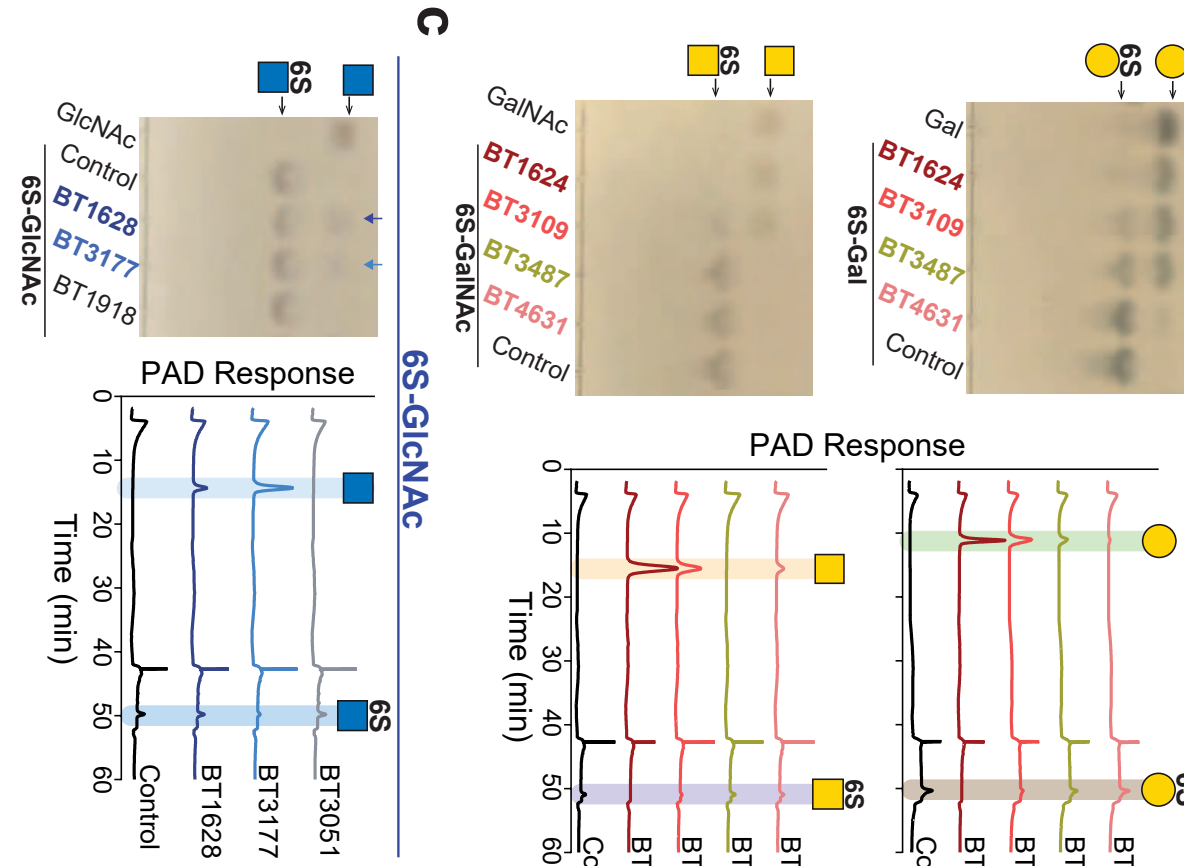

o

$\sigma$

4S-Gal/GalNAc
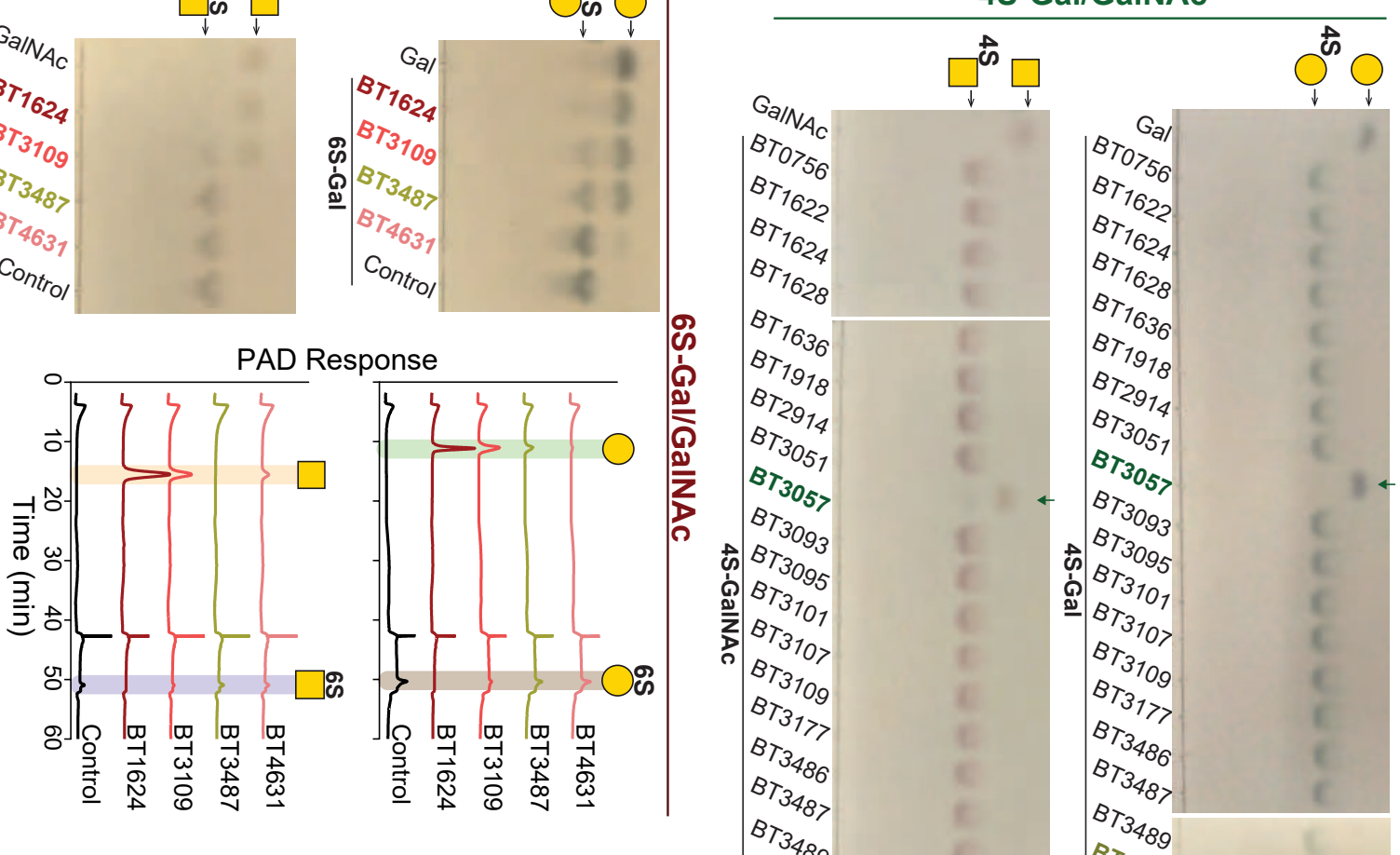

으
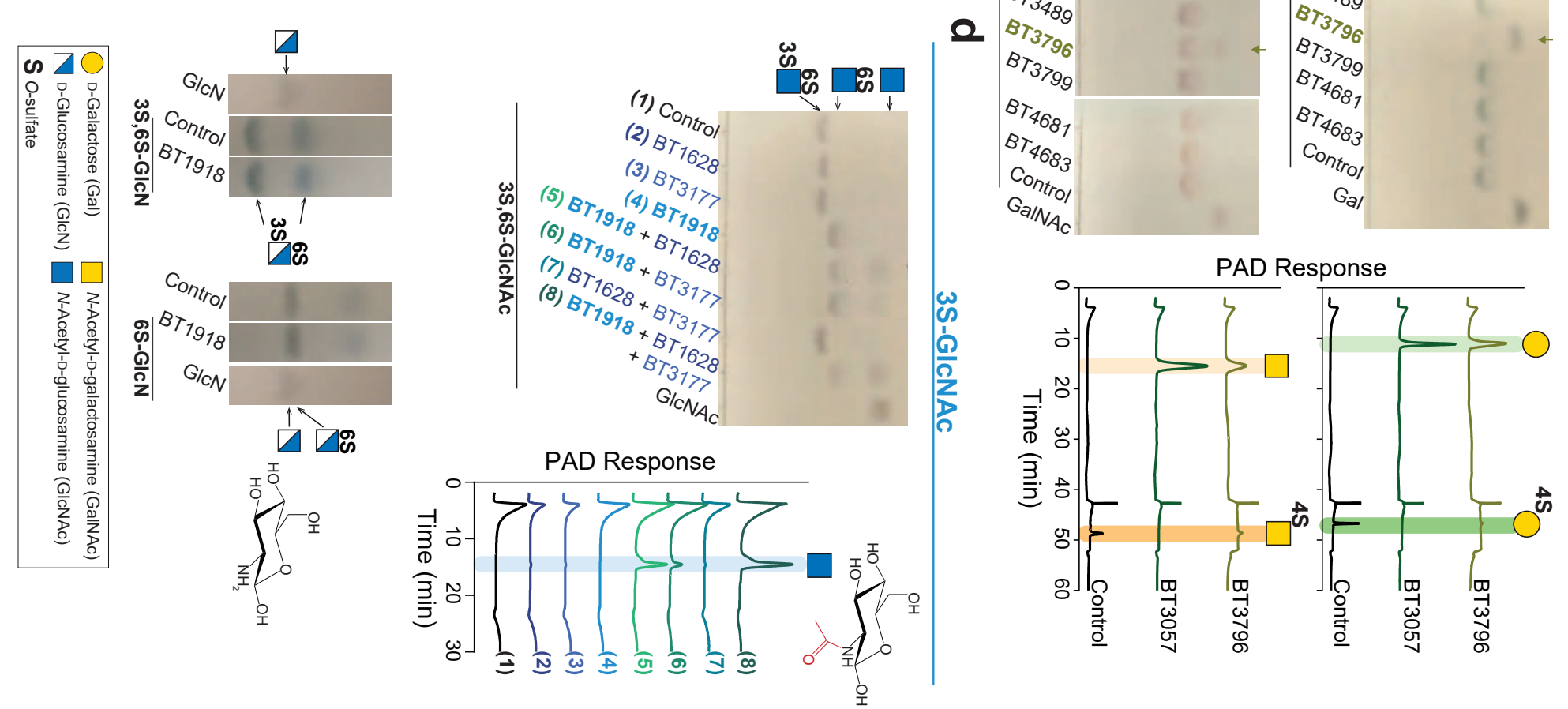
a

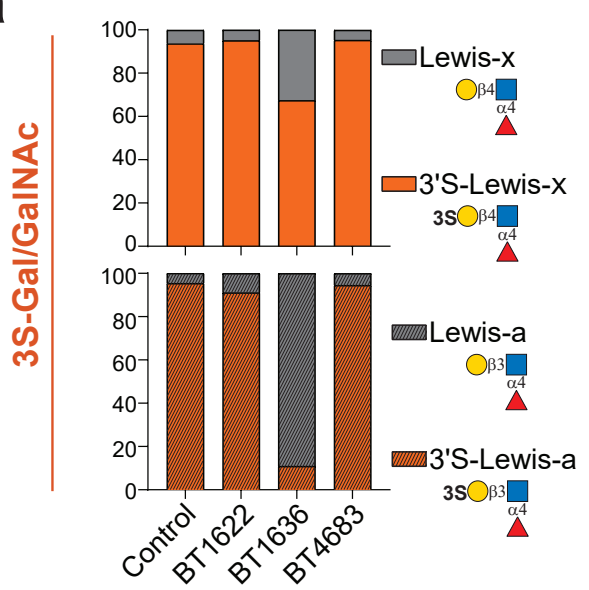

茯|

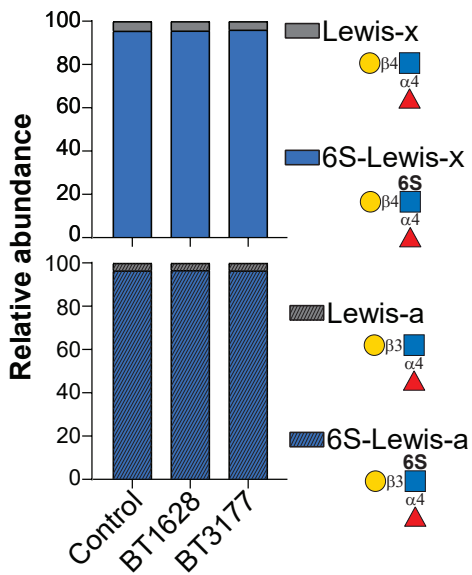

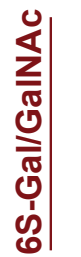

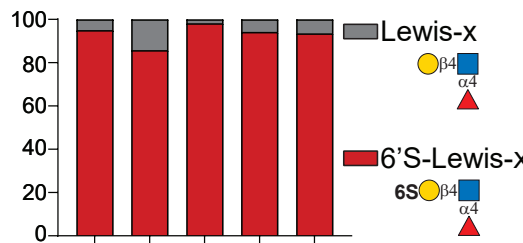

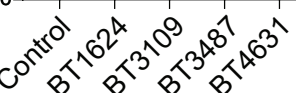

C

3S-Gal/GalNAc

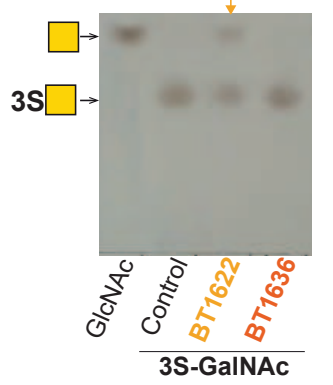

b 3S-Gal/GalNAc
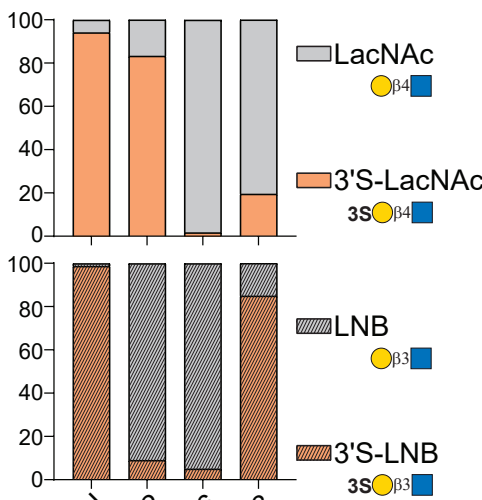

एक $6^{2} 6^{30} 0^{8^{3}}$
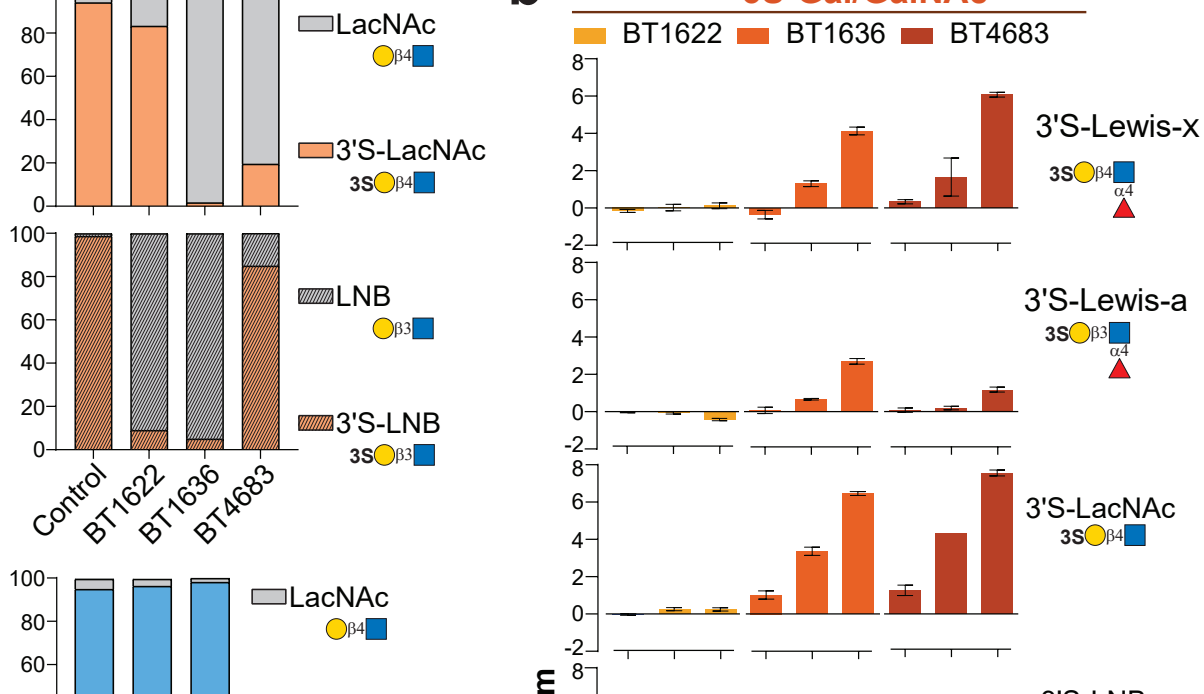

3'S-Lewis-a

$35 \bigcirc \beta^{3}$

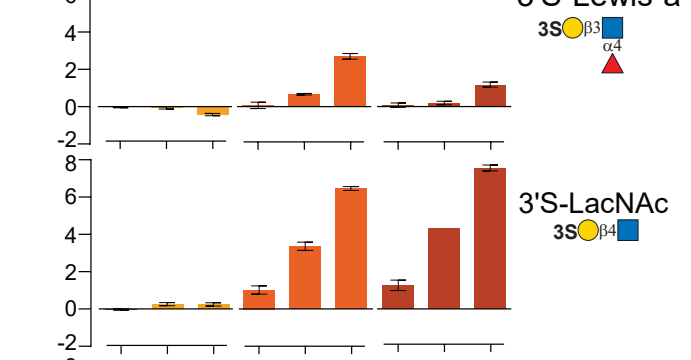

$\varepsilon^{8}$
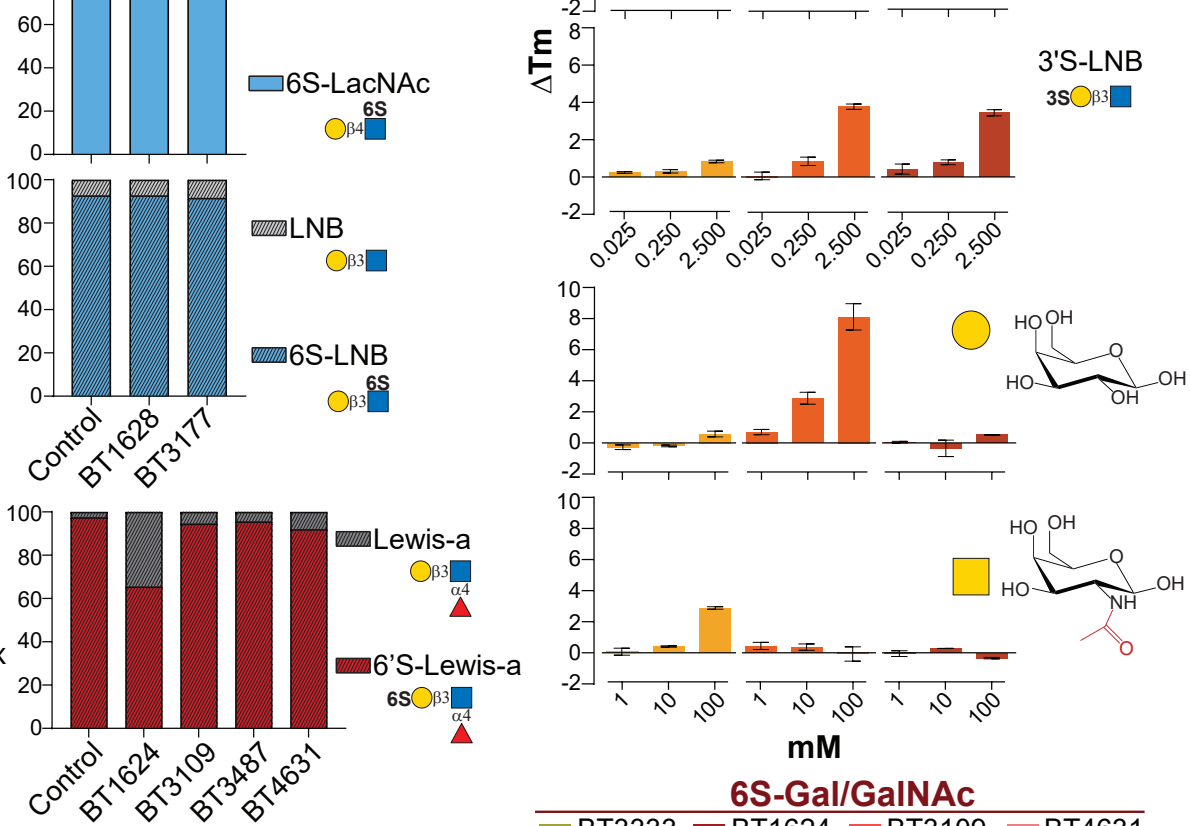

$\stackrel{\alpha^{4}}{\triangle}$

6S-Gal/GalNAc
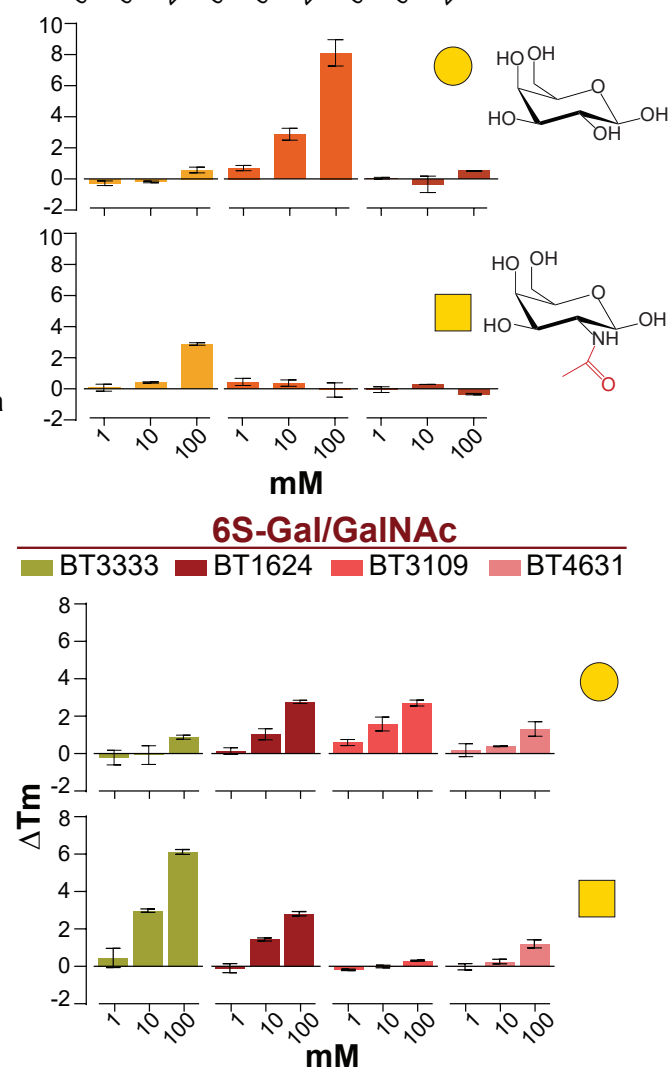
a
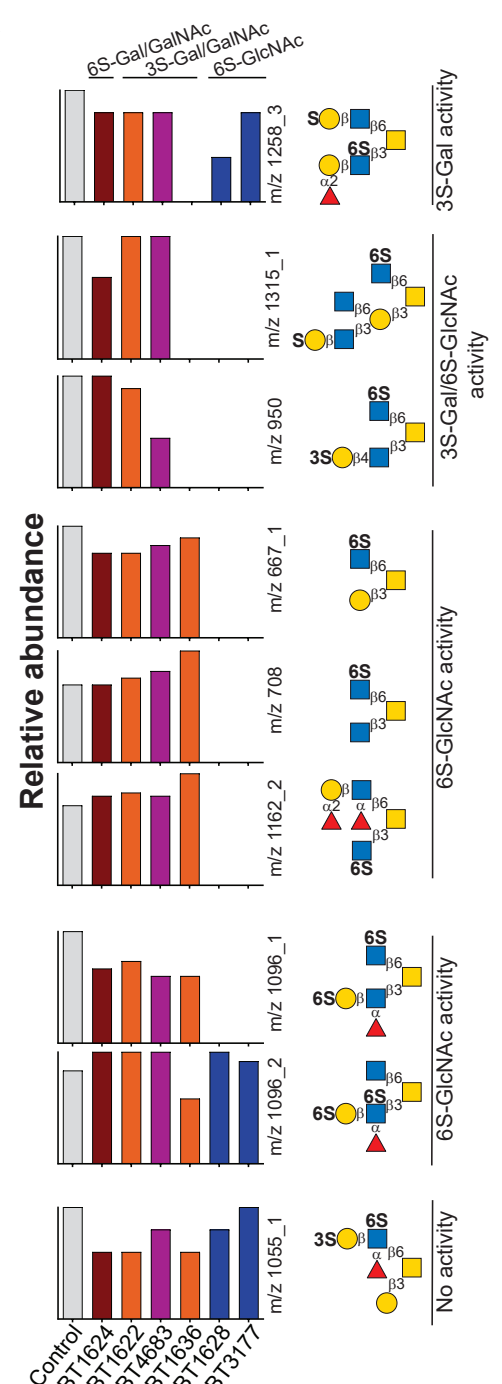

b

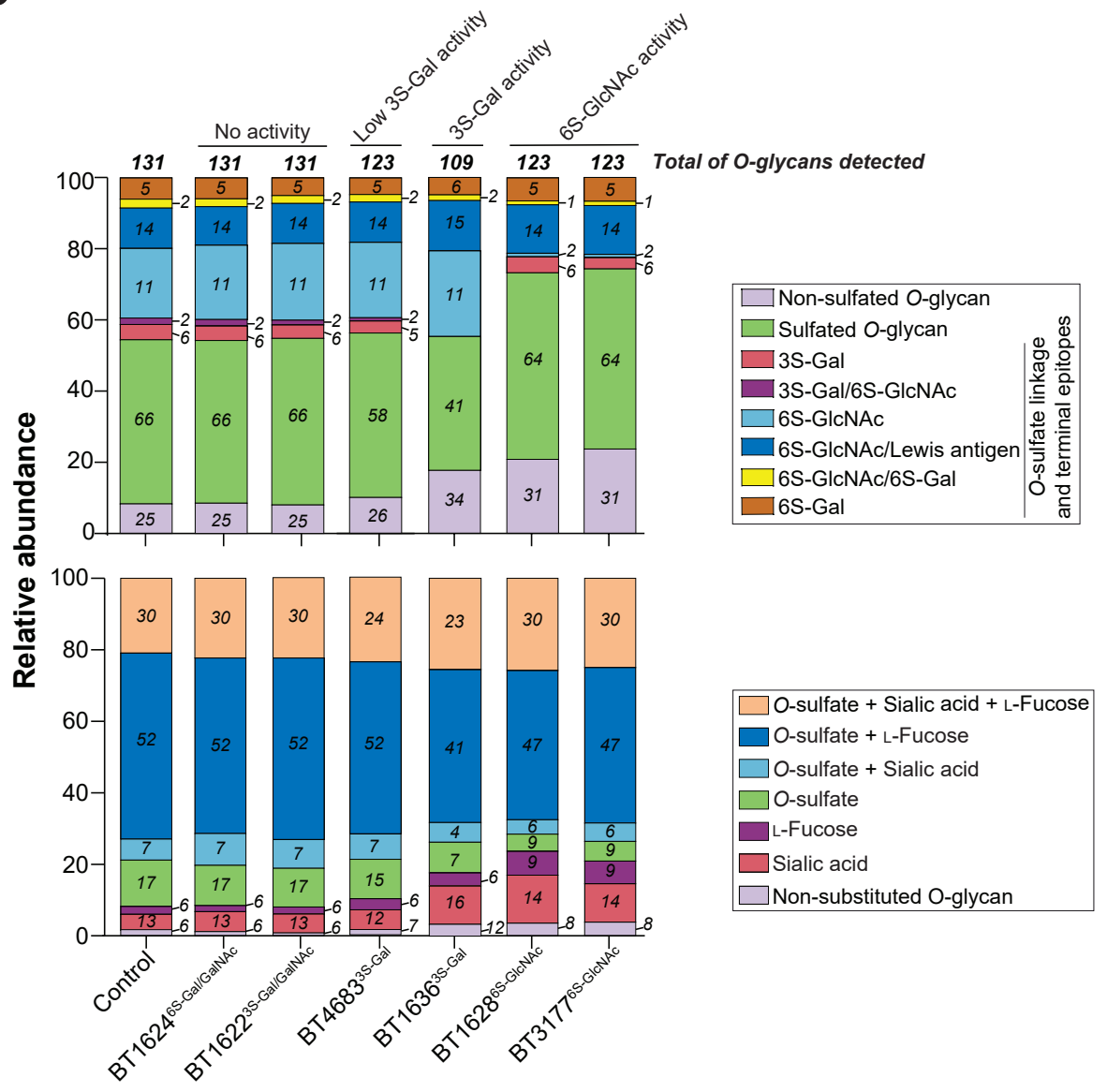

S O-sulfate $\triangle$ L-Fucose $\bigcirc$ D-Galactose $(\mathrm{Gal})$ N-Acetyl-D-glucosamine (GlcNAc)

$\square$ N-Acetyl-D-galactosamine (GalNAc)

Sulfatase subfamily: $\square$ S1_4 $\square$ S1_11 $\square$ S1_16 $\square$ S1_20 
a

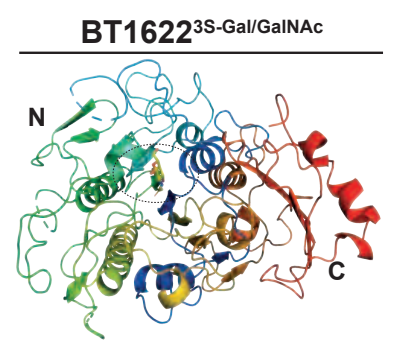

๓

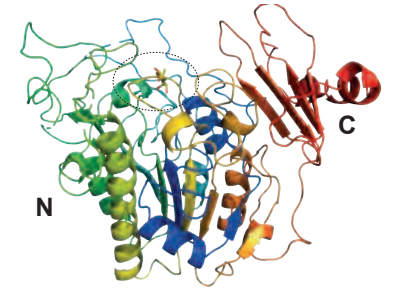

d
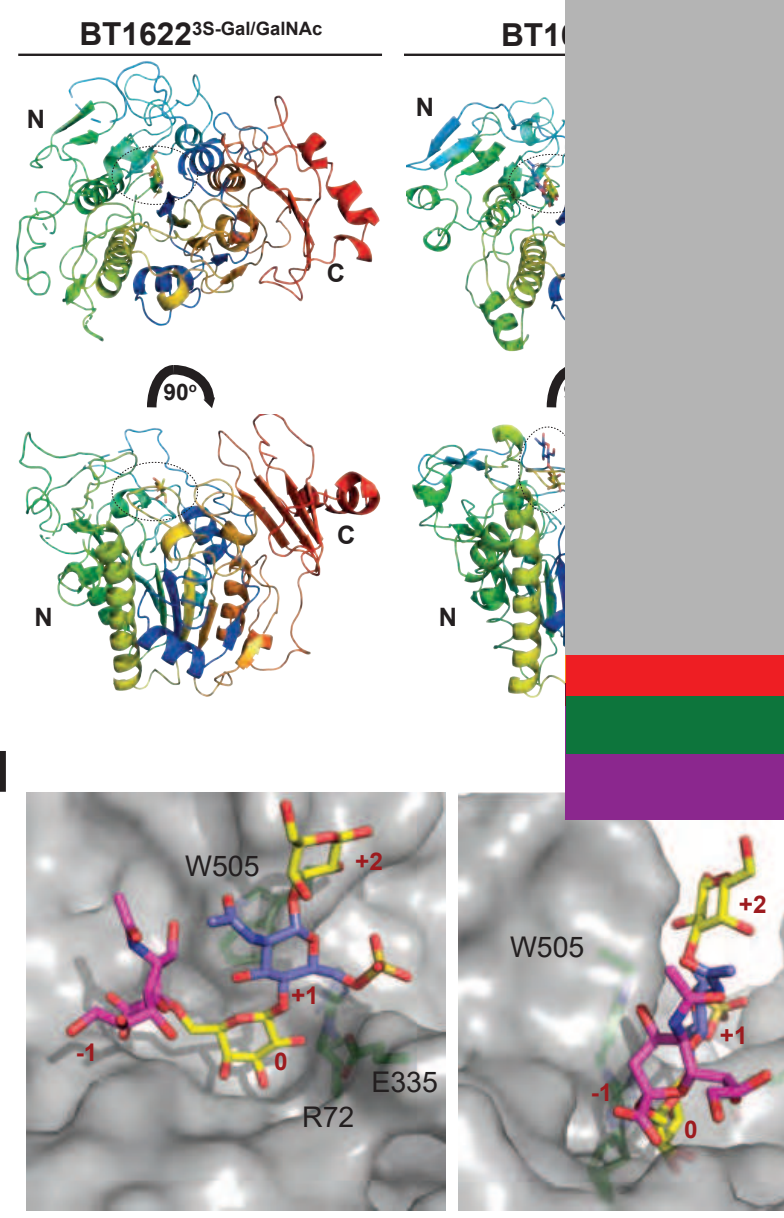

(90)
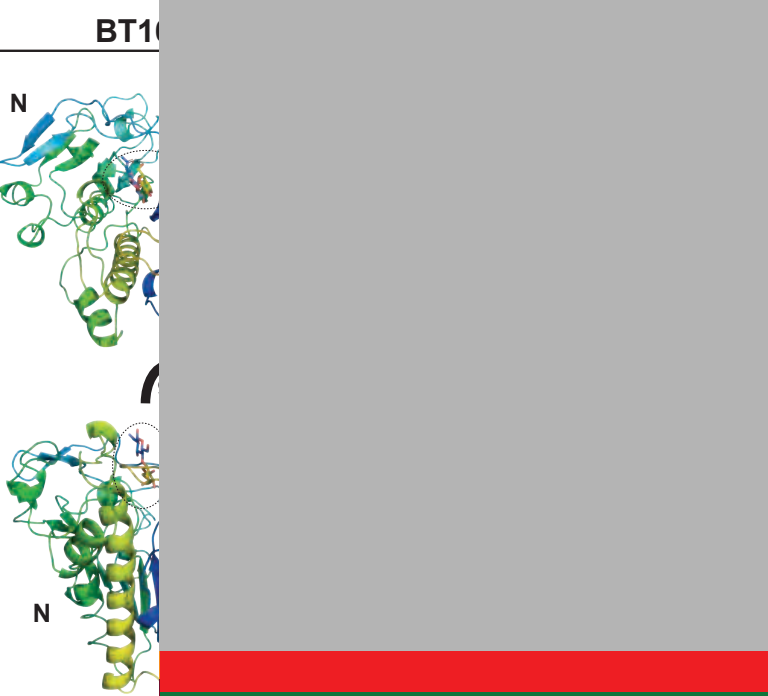

(9)
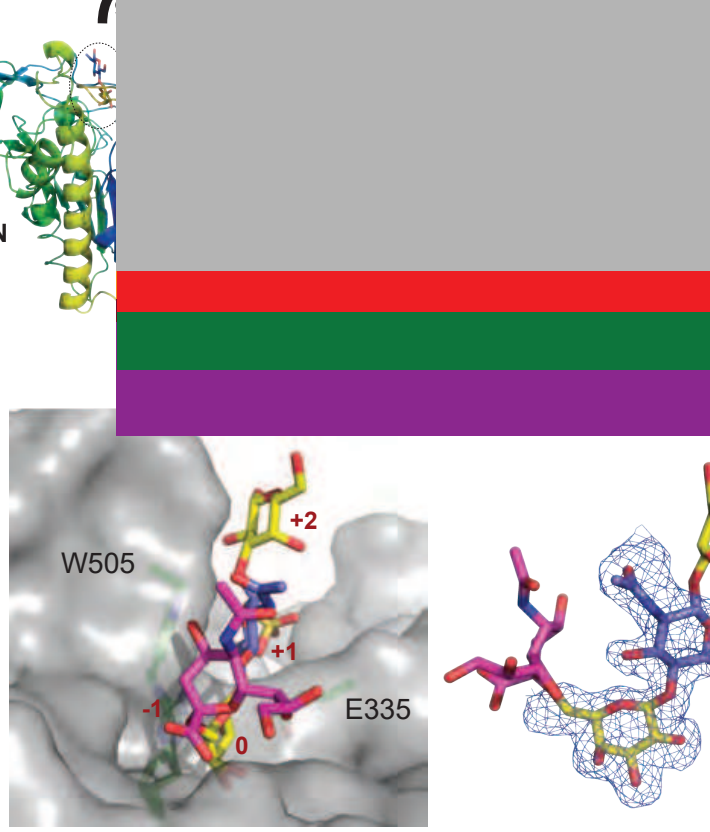

b

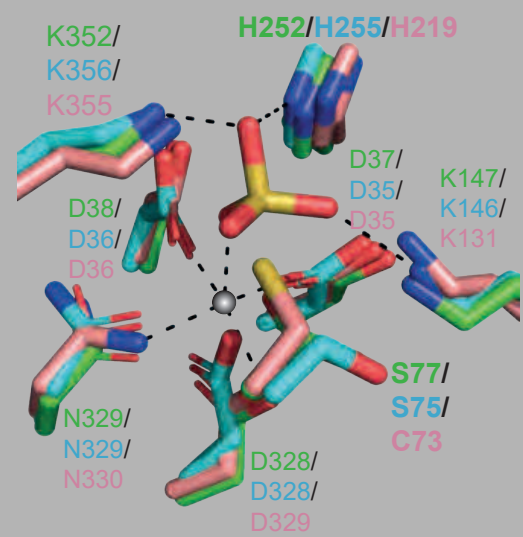

C

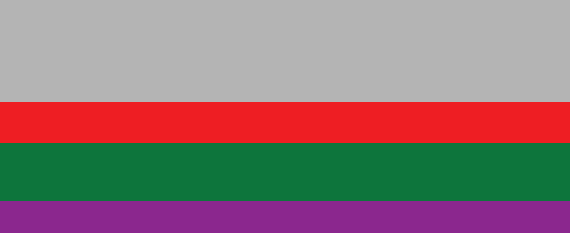

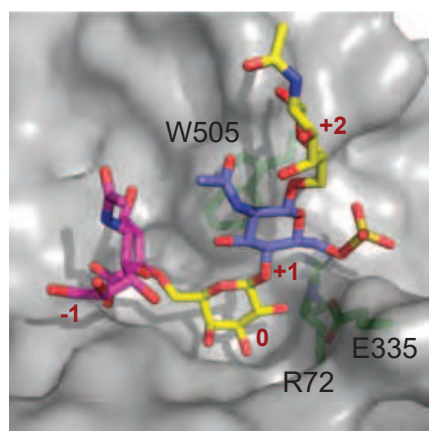

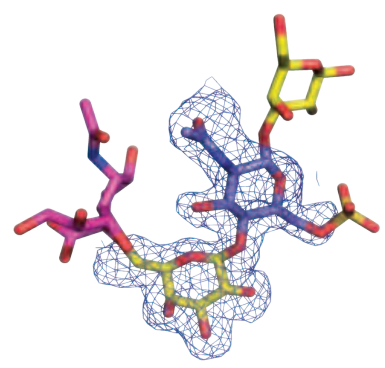

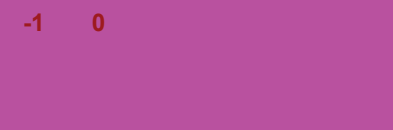
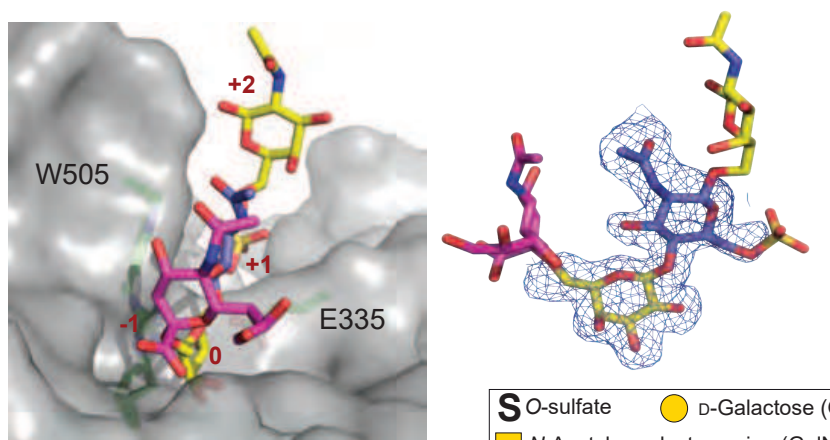

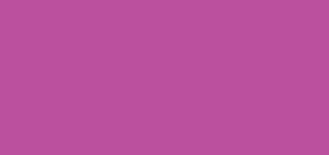

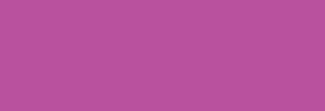

S O-sulfate D-Galactose (Gal) $\square$ N-Acetyl-D-glucosamine (GICNAc) $\square$ N-Acetyl-D-galactosamine (GaINAc) $>N$-Acetylneuraminic acid 
a

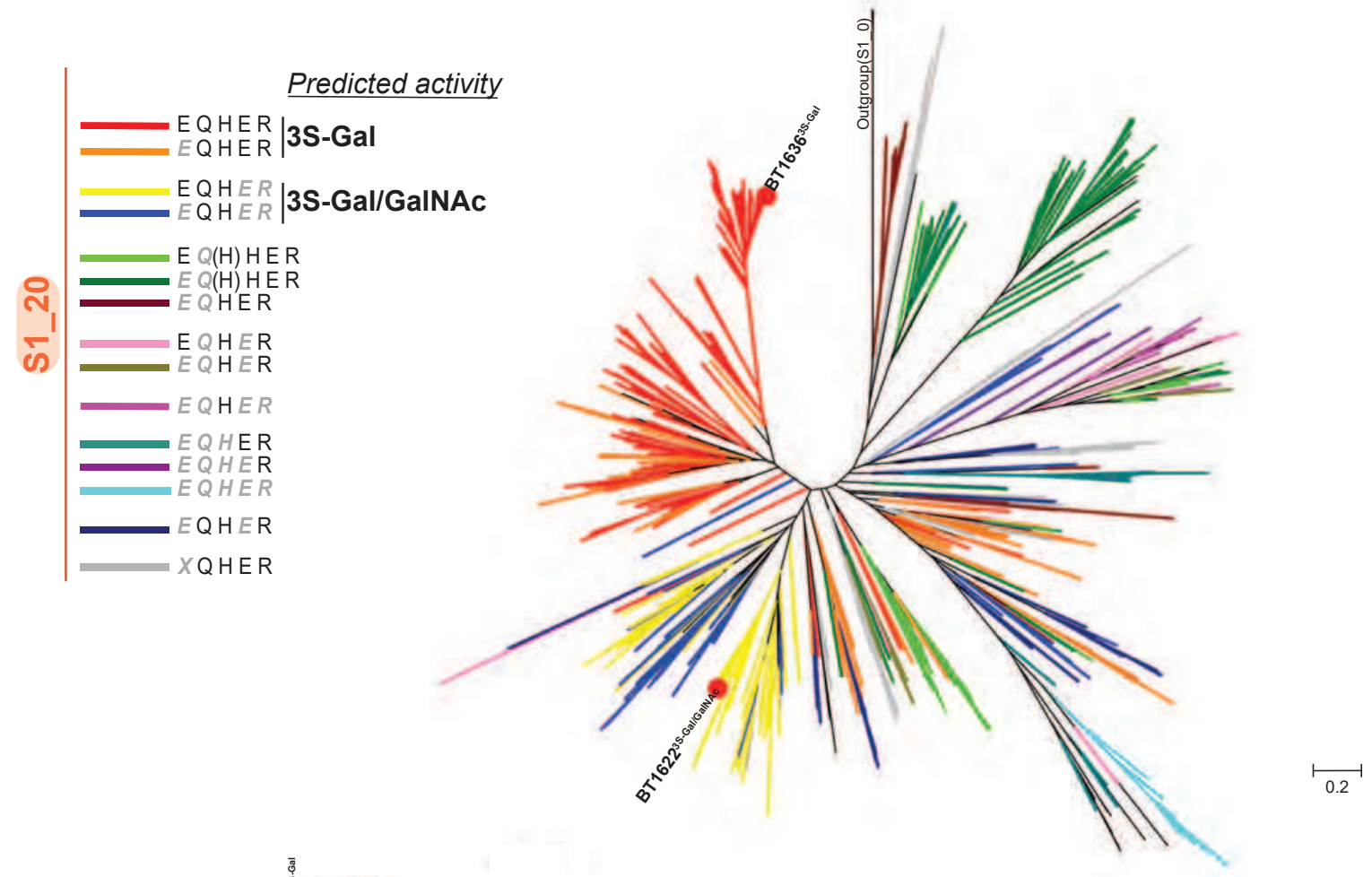

b

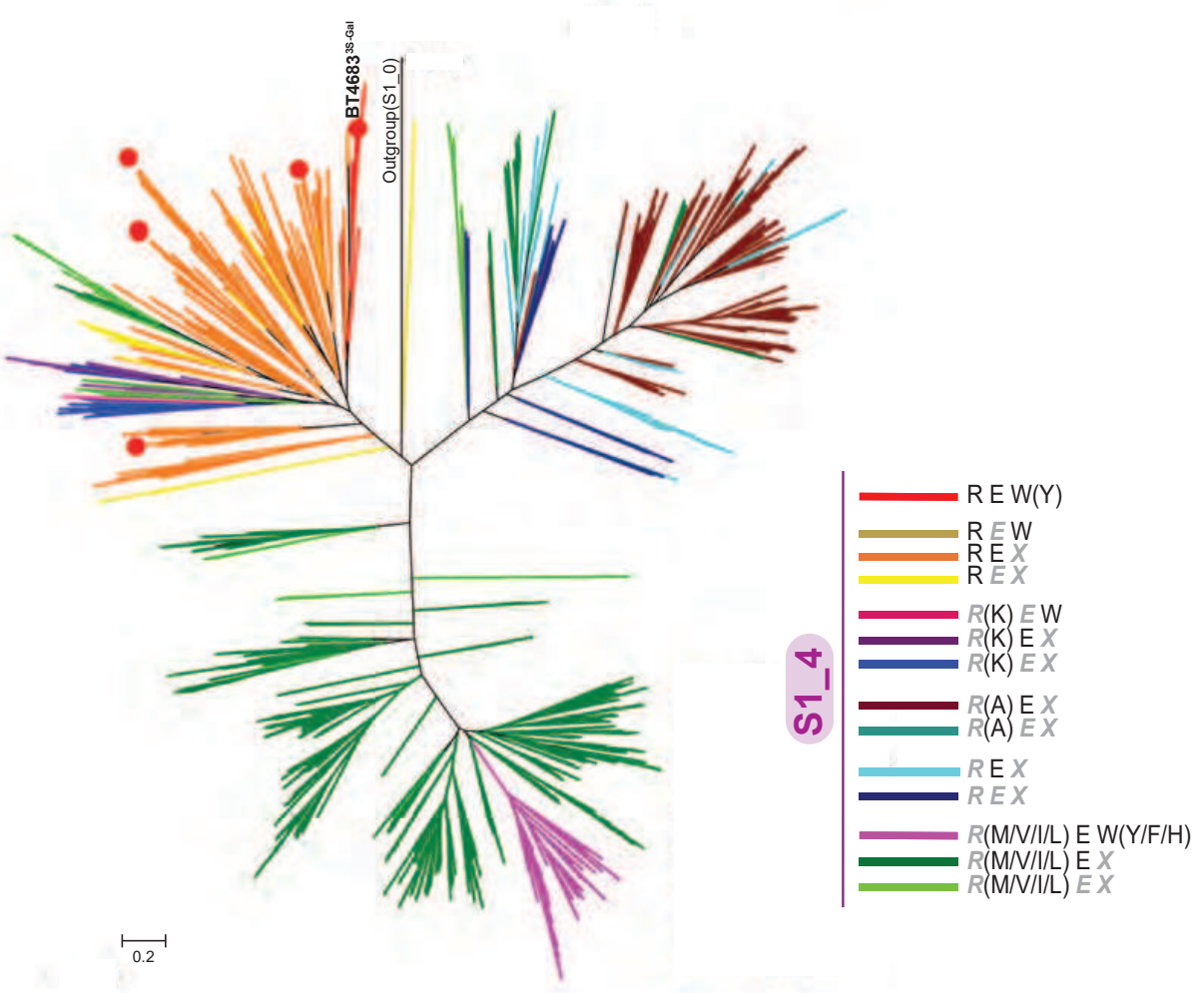


a

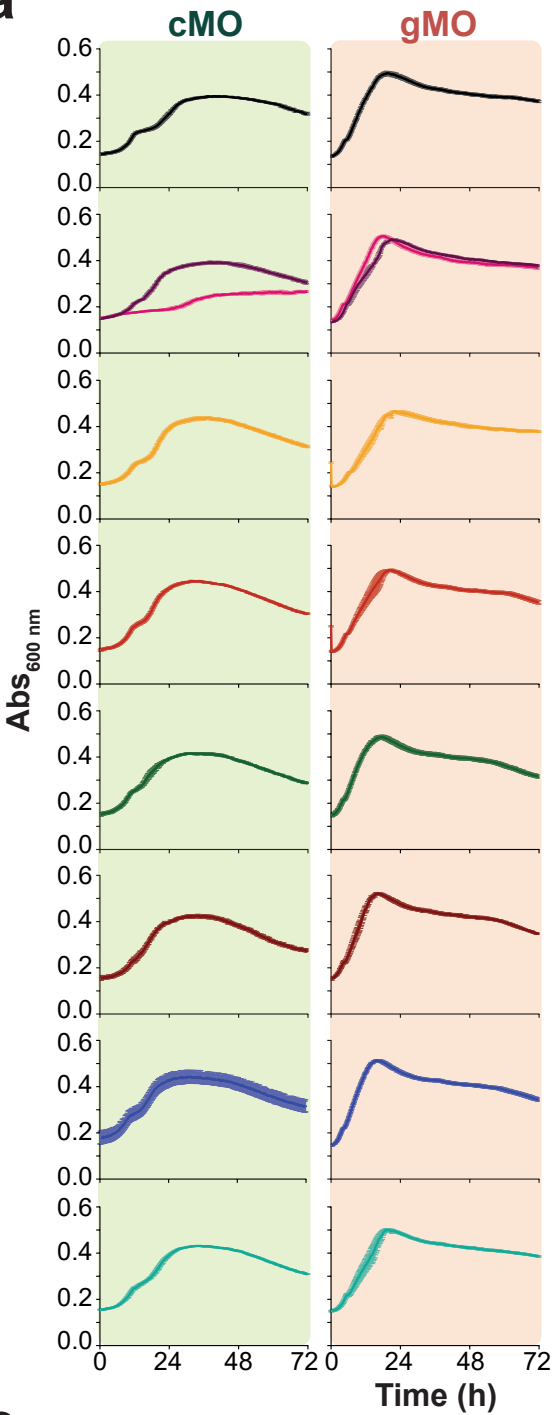

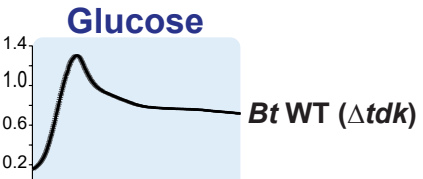
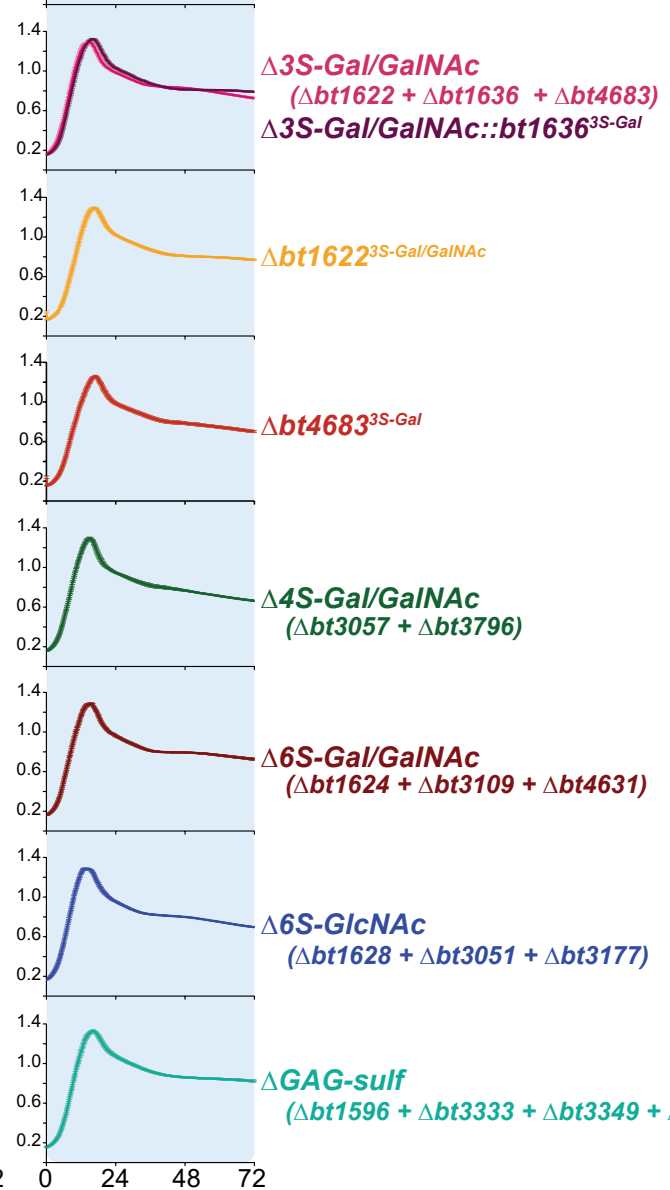

$\triangle 6 S-G I c N A C$

$(\Delta b t 1628+\Delta b t 3051+\Delta b t 3177)$

$\triangle G A G-s u l f$

$(\Delta b t 1596+\Delta b t 3333+\Delta b t 3349+\Delta b t 4656)$ b

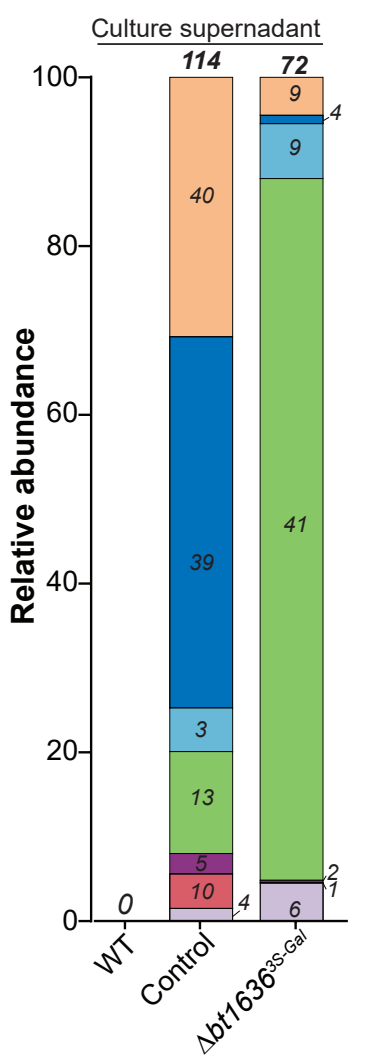

$\square$ O-sulfate + Sialic acid + L-Fucose $\square$ O-sulfate + L-Fucose $\square$ O-sulfate + Sialic acid $\square$ O-sulfate $\square$ L-Fucose $\square$ Sialic acid

$\square$ Non-substituted O-glycan
C

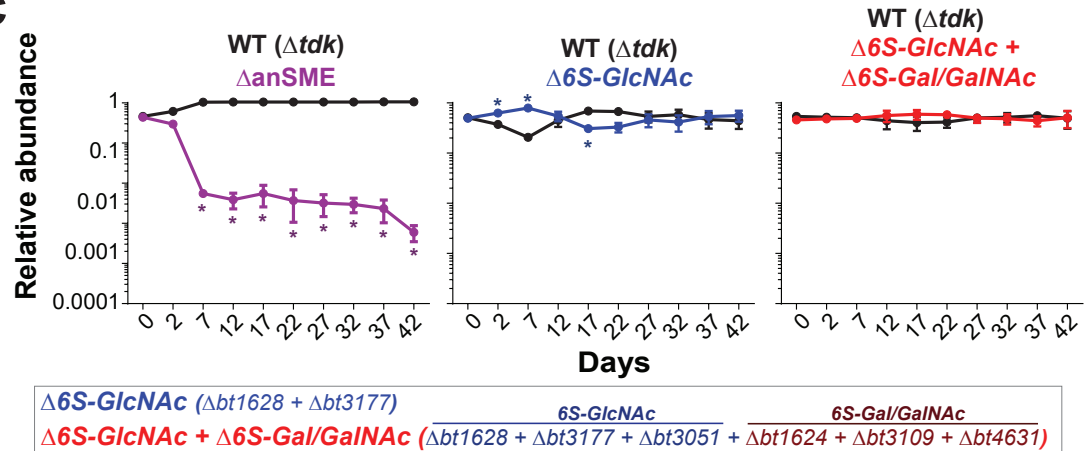

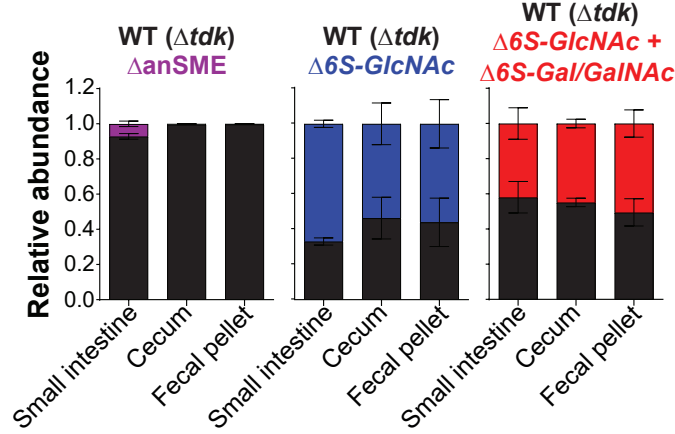




\section{Supplemental Discussion}

\section{Utilization of different mucin O-glycans sources by HGM}

Mucin composition varies throughout the gastrointestinal ( $\mathrm{Gl}$ ) tract, with the stomach having mainly MUC5AC and the colon mainly MUC2 ${ }^{1}$. The glycosylation of these respective mucins also varies along the $\mathrm{Gl}$ tract with higher levels of sulfated and sialylated structures observed in the distal colon compared to the upper GI tract ${ }^{2}$. Among the 20 bacterial strains tested for growth, 12 failed to grow on gastric mucin O-glycans (gMO) or colonic mucin O-glycans (cMOs) (Fig. 1b). Only 6 bacteria were able to utilize both O-glycans substrates but growth was variable. In both O-glycan substrates, Bacteroides thetaiotaomicron (Bt), B. caccae, B. fragilis and B. fluxus grew better than B. dorei and B. vulgatus (Fig. 1b and Extended Data Fig. 1). The differences observed in the growth profiles were reproducible in two different batches of purified cMOs (Fig. 1b) Indeed, it is likely that different HGM members have evolved to target different (or only a subset) of the available O-glycans and this fine-tuning of host glycan utilization may have important implications in gut colonization and symbiosis. Additionally, B. massiliensis and Akkermansia mucinipila grew on gMO but failed to utilize cMO (Fig. 1b and Extended Data Fig. 1). Both strains were able to grow on $\mathrm{N}$-acetylglucosamine (GlcNAc) and Akkermansia mucinipila grew on GlcNAc in the presence of cMO suggesting that these O-glycans do not inhibit the growth of this bacterium. Previous studies have determined that $B$. massiliensis and Akkermansia mucinipila are mucin-degraders by demonstrating growth on gastric mucins $^{3,4}$. However, the lack of growth in colonic O-glycans suggests that these bacteria are not able to initiate the degradation of more complex, sulfated colonic glycans. This finding highlights the importance of taking into account O-glycosylation differences along the $\mathrm{Gl}$ tract and the need to utilize colonic mucins to draw conclusions regarding the full mucin-degrading potential of the colonic HGM.

\section{Sulfatase activity in $\mathrm{cMO}$}

Despite all 12 sulfatases being active on defined oligosaccharides, of those tested on cMO, BT1622 ${ }^{3 S-G a l / G a l N A c}$ (S1_20 subfamily) and BT1624 ${ }^{6 S-G a l / G a l N A c}$ (S1_15 subfamily) did not show any activity on this complex substrate (Fig. 2, Extended Data Fig. 5 and Supplementary Table 4). These findings are consistent with the results 
observed in defined commercial oligosaccharides where BT1622 3 S-Gal/GalNAc showed a preference for sulfated GalNAc over Gal glycans (Extended Data Fig. 4) and BT1624 ${ }^{6 S-G a l / G a l N A c}$ activity is blocked by the presence of additional substitutions (such as Lewis antigens) (Extended Data Fig. 4a). Additionally, 2 of 6 detected 6S-Gal structures contained a capping sialic acid and a terminal blood group $\mathrm{H}$ type 2 [Fuc-

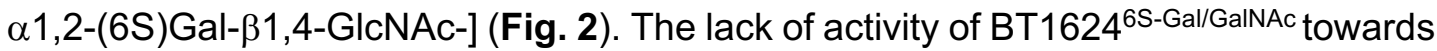
such structures confirms an exo-mode of action that we describe for this sulfatase using commercial substrates.

Overall, when compared to the non-enzyme treated control, we detected an increase of non-sulfated structures and decrease of sulfated oligosaccharides in all samples with the active enzymes (BT4683 ${ }^{3 S-G a l}$, BT1636 3 S-Gal, BT1628 ${ }^{6 S-G I c N A c}$ and BT3177 ${ }^{6 S-G I C N A c}$ ) (Extended Data Fig. 5b). BT1636 3 -Gal (S1_20) was active towards all detected 3S-Gal structures with the exception of glycan 1055_1 that is a doubly sulfated 3S-Gal/6S-GlcNAc fucosylated structure (Extended Data Fig. 5a). As we observed using commercial substrates, the presence of Lewis-a/x epitopes leads to a decrease in the activity of this sulfatase (Extended Data Fig. 4a and Supplementary Table 4) and the presence of a second sulfate group might exacerbate this negative effect leading to the lack of activity towards this complex sulfated O-glycan. The incubation of the 6 S-GIcNAc sulfatases BT1628 ${ }^{6 S-G I C N A c}$ and BT3177 ${ }^{6 S-G I C N A c}$ with cMO suggests that these enzymes are redundant, but because they are encoded in different PULs they could be expressed in response to different activating cues (Fig. 2 and Supplementary Table 4). Compared to the non-enzyme treated control, 16 glycans were not detected after incubation with these sulfatases, 14 of these structures have a terminal 6S-GICNAc (Fig. 2 and Supplementary Table 4). BT16286S-GICNAc and BT3177 ${ }^{6 S-G I c N A c}$ were active in 6 S-GIcNAc core 3 (GIcNAc- $\left.\beta 1,3-G a l N A c\right)$ and core 4 (GlcNAc- $31,6-$-GalNAc) structures (Extended Data Fig. 5a), suggesting that these sulfatases are well suited to accommodate the variations in linkages/sugars found in mucin O-glycans. Additionally, we also detect 7 new glycans that are likely to be reaction products of BT1628 ${ }^{6 S-G I C N A c}$ and BT3177 6 S-GlcNAc (Fig. 2 and Supplementary Table 4).

The identification and characterization of the first sulfatases active on mucin $O$ glycans creates the opportunity to improve our understanding of O-glycan structures by using these enzymes as analytical tools. After the treatment with BT1636 3 S-Gal 
several oligosaccharides predicted to contain a terminal sulfate linked to Gal were not detected. Although we could not determine the specific sulfate linkage by mass spectrometry, the activity of the 3S-sulfatase suggests that these oligos contain a terminal 3S-Gal (Extended Data Fig. 5a and Supplementary Table 4). The specificity of the 6S-GICNAc sulfatases for non-fucosylated O-glycans also illuminates their potential use as tools to characterize the structure of these complex structures since it allows the differentiation of different isomers. For example, we detect two oligosaccharides with mass 1096 , however, after incubation with BT1628 ${ }^{6 S-G I C N A c}$ or BT31776S-GICNAc, only the isomer 1096_2 was detected, indicating that the isomer 1096_1 contain a terminal 6S-GIcNAc (Extended Data Fig. 5a and Supplementary Table 4).

\section{Conserved structural features of the $\mathbf{S 1}$ formylglycine family}

\section{Protein fold and subsites nomenclature}

S1 sulfatases comprise the most common and largest family of sulfatases, currently encompass 36,816 members in sulfAtlas and are found in all domains of life ${ }^{5}$. S1 sulfatases are part of the alkaline phosphatase superfamily and adopt an alkaline phosphatase-like fold. This is an $\mathrm{N}$-terminal $\alpha / \beta / \alpha$ domain with $\mathrm{S} 1$ sulfatases also possessing a smaller C-terminal 'sub domain'. The active site is located in the $\mathrm{N}$ terminal domain that has a large mixed $\beta$-sheet composed of $\sim 10 \beta$ strands, sandwiched between $\alpha$ helices above and below. The C-terminal 'sub-domain' is composed of a 4 stranded antiparallel $\beta$-sheet and a single amphipathic terminal helix.

This $\mathrm{C}$-terminal domain abuts the $\mathrm{N}$-terminal domain through the antiparallel $\beta$-sheet with loops from the $\beta$ strands sometimes contributing to the active site architecture (Extended data Fig. 6a). The subsite nomenclature for carbohydrate sulfatases is such that the invariant sulfate binding site is denoted as the $S$ site. The $S$ site sulfate is appended to the 0 subsite sugar. Subsites then increase in number (i.e. $+1,+2,+3$ ) as the sugar moves toward the reducing end (free 01 ) and decreases in number as the sugar chain moves towards the non-reducing end (i.e. $-1,-2,-3)^{6}$. 


\section{S1 formylglycine active site conservation}

The sulfate binding site ( $\mathrm{S}$ site) is invariant across the $\mathrm{S} 1$ family and comprises the catalytic residues (nucleophile and catalytic acid) and a calcium binding site (Extended data Fig. 6b). An invariant histidine is likely the potential catalytic acid but a lysine has also been suggested to possibly fulfil this role ${ }^{7}$. The pKa of His is $\sim 6.0$, whilst Lys has a pKa of $>10$, making it more chemically feasible that His performs the role of the catalytic acid. Homologues of these residues (H252 and K352 in BT1636 35 Gal) make hydrogen bonds to the scissile sulfoester linkage (Extended data Fig. 6b). Previously published work with BT1596 and BT4656, which are 2S-Uronic acid and 6S-GIcNAc sulfatases, respectively, showed that the mutation of either residue to alanine causes inactivation ${ }^{7}$. Consistent with this work, a BT4683 ${ }^{3 S-G a l}$ H219A mutant was inactive. However, in BT1622 ${ }^{3 S-G a l / G a l N A c}$, the mutation of $\mathrm{H} 255$ to Ala caused only a $\sim 30$-fold decrease in activity (Supplementary Table 3). Thus, it is possible that in BT1622 ${ }^{3 S-G a l / G a l N A c}$ the loss of $\mathrm{H} 255$ is compensated by the invariant residue $\mathrm{K} 356$ and interestingly BT1622 $2^{3 S-G a l / G a l N A c}$ has a pH optimum $\sim 2$ units higher than most sulfatases assayed (Supplementary Fig. 4).

The calcium binding site is located at the base of the $S$ site interacting with the sulfate group. This calcium ion is an essential component of the catalytic mechanism helping to stabilise negative charges that occur during the catalysis. All three of the solved structures had occupation for calcium. In BT1636 3 -Gal D328 and the sulfate group of the substrate coordinate above and below the calcium with D37, D38, N329 and the formylglycine binding in a plane completing an octahedral coordination (Extended Data Fig. 6b). These three Asp and the Asn coordinated with calcium are structurally conserved in all 3S-Gal/GalNAc sulfatases structures (Extended data Fig. 6b).

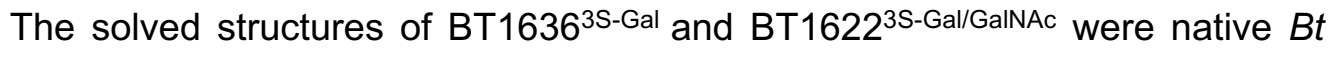
proteins having a Ser at the formylglycine position. However, the structure of BT46833S-Gal was obtained with the active protein where S73 was mutated to Cys (as E. coli can only convert Cys, not Ser, to formylglycine). The analysis of BT4683 ${ }^{3 \mathrm{~S}-\mathrm{Gal}}$ reveals that the crystallized protein still has the Cys and not formyglycine indicating poor installation of the formylglycine. This observation means the kinetic data, (although the rates are significant and readily measurable) may be an underestimation of true catalytic performance. This will affect the $k_{\text {cat }}$ component of the $k_{\text {cat }} / K_{\mathrm{M}}$ 
measurement and thus the $k_{\mathrm{cat}} / K_{\mathrm{M}}$ reported in Supplementary Table 3 is an underestimate of the true activity.

\section{Additional 3S-Gal/GalNAc specificity determinants based on structures}

BT1636 ${ }^{3 S-G a l}$ was solved in complex with the product LacNAc; the Gal at 0 subsite is well ordered and makes extensive interactions, whilst the +1 GlcNAc is highly disordered and appears to make no interactions with the protein (Fig. 3 and Extended Data Fig. 6c). $\mathrm{O} 2$ of the Gal hydrogen bonds with $\mathrm{O} \varepsilon 1$ of $\mathrm{E} 334$ and $\mathrm{NH} 2$ of R353. Mutation of these residues to Ala causes $\sim 300$ and $\sim 60$-fold reductions in $k_{\text {cat }} / K_{\mathrm{M}}$, respectively. The $\mathrm{O} 6$ group of Gal potentially coordinates with $\mathrm{O} \varepsilon 2$ of E100 and $\mathrm{N} \varepsilon 2$ of $\mathrm{Q} 173$ and mutations of these residues to Ala cause $\sim 80$ and $\sim 50$-fold decreases in $k_{\text {cat }} / K_{\mathrm{M}}$, respectively (Fig. 3 and Supplementary Table 3 ). Comparison of the BT1622 ${ }^{3 S-G a l / G a N A c}$ structure with BT1636 ${ }^{3 S-G a l}$ shows that E98 and Q172 (which correspond to E100 and Q173 in BT1636 ${ }^{3 S-G a l}$ ) are conserved (Fig. 3) and mutating E98 to Ala caused only a 15 -fold decrease in $k_{\text {cat }} / K_{M}$ (Supplementary Table 3 ). Additionally, in BT1622 3 S-Gal/GaNAc the hydrophobic interactions with the $N$-acetyl group, and the more open pocket, may offset the effects H176A (300-fold loss in activity) when compared to H177A (complete loss in activity) in BT1636 ${ }^{3 S-G a l}$ (Fig. 3 and Supplementary Table 3).

BT4683 ${ }^{\text {Gal-3S }}$ also displayed the same 3S-Gal activity as the S1_20 enzymes but showed a preference for 3'S-LacNAc, reciprocal to BT1622 ${ }^{3 S-G a l}$. BT46833S-Gal bound the $\mathrm{O} 2 \mathrm{Gal}$ of LacNAc via Oع2 of E335 (equivalent to E334 in BT1636 ${ }^{3 \mathrm{~S}-\mathrm{Gal}}$ ) and through either $\mathrm{N} \varepsilon$ or $\mathrm{NH} 1$ of $\mathrm{R} 72$ (Fig. 3). Although R72 is sequentially distal to R353 in BT1636 35 -Gal it is spatially similar and likely contributes in a similar capacity (Fig. 3). Despite the mutations $\mathrm{R} 72 \mathrm{~A}$ and E335A resulting in loss of activity, the Glu and Arg are only conserved in $62 \%$ and $19 \%$ of S1_4 sequences, respectively, suggesting there is a significant but not absolute selection for an equatorial $\mathrm{O} 2$ in this subfamily (Extended Data Fig. 7 and Supplementary Fig. 2). Uniquely among the 3S-Gal sulfatases identified, BT4683 3 S-Gal utilises a hydrophobic stacking interaction through W505 to provide a platform for the +1 GlcNAc and partially the 0 Gal. Mutation of W505 to Ala almost completely abolishes activity on 3'S-LacNAc (Supplementary Table 3) but surprisingly this residue is not conserved in our phylogenetic analyses of S1_4 being present in only 8 other sequences (Extended Data 7 and Supplementary 
Fig. 2). It is important to note, that W505 is not well conserved; potential equivalent aromatic residues can be found in some additional clades, which are coloured light brown (or bronze), pink or dark red, but it is not evident from the alignment that these are functional equivalents. Future structural work is needed to confirm if other aromatic residues take equivalent positions in those sulfatases. Additionally, the BT468335-Gal activity against defined sulfated saccharides was suggestive of an exo-acting enzyme that cleaves terminal 3S-Gal (Extended Data Fig. 4). However, a close analysis of this sulfatase structure shows that the active site is located in an open cleft characteristic of an endo-active enzyme ${ }^{6}$. This more open cleft of BT4683 ${ }^{3 S-G a l}$ may allow additional sugars/sulfates to be accommodated on the $\mathrm{O} 6$ of both the $0 \mathrm{Gal}$ and +1 GlcNAc. Indeed, the activity determined in cMO shows that this sulfatase can act on sialylated O-glycan (Fig. 2). Further modelling of different O-glycan structures (using the crystallographically solved LacNAc as an 'anchor') indicate that this enzyme can accommodate complex O-glycans with internal sulfation (Fig. 3 and Extended Data 6d). Together, these results suggest that BT4683Gal-3S, and its close homologues, could be endo $3 S$ sulfatases where the 0 subsite specificity for $G$ al is driven by glycan context and/or distal subsites such as -1 and +2 , rather than an axial O4 as in S1_20.

Additionally, it is unclear why BT1636 ${ }^{\text {Gal-3S }}$ acts better on LacNAc substrates than BT1622 ${ }^{3 \mathrm{~S}-G a l / G a l N A c}$. It is interesting to note, however, that both BT1636 ${ }^{3 \mathrm{~S}-G a l}$ and BT4683 ${ }^{3 \mathrm{~S}-G a l}$ perform well on LacNAc configured substrates and utilise an Arg and Glu to coordinate $\mathrm{O} 2$ whilst BT1622 ${ }^{3 \mathrm{~S}-\mathrm{Gal}}$ lacks these residues (Fig. 3). These residues may lead to the enhanced activity on LacNAc ( $\beta 1,4$ glycan) vs. LNB ( $\beta 1,3$ substrate). Another thing to note is that a $\beta 1,4$ vs $\beta 1,3$ linkage will rotate the GIcNAc $\sim 60^{\circ}$ but switch the position of the Fuc residue from being on the ' $N$-acetyl side' of the glycosidic bond to the 'O6 side' of the glycosidic bond, and this may also be the cause of the differential activities on $\beta 1,4$ vs $\beta 1,3$ linked substrates.

\section{Phylogenetic analyses of S1 20 specificity determinants}

The essential His that acts as a key specificity determinant of galacto- over gluco-substrates (H177 and $\mathrm{H} 176$ in BT1636 ${ }^{3 S-G a l}$ and BT1622 ${ }^{3 S-G a l / G a l N A c}$, respectively) is highly conserved (92\% of S1_20 sequences) (Extended data Fig. 7 and Supplementary Fig. 3). The GIn (Q173 and Q172 in BT1636 ${ }^{3 S-G a l}$ and BT1622 35 - 
Gal/GalNAc, respectively) is only conserved in $66 \%$ of sequences and in $25 \%$ of the cases is substituted with a histidine, a residue that can also fulfill the same role of GIn interacting with Gal O6. Indeed, these conserved residues are located in a highly conserved domain with the consensus sequence [CDNS]-[QH]-[RVF]-[QHLD]-[AG]-H[NRST]-[YHF]-[YF]-P (Prosite syntax). With H177 targeting the axial O4 of Gal directly, a Q173 may function indirectly to select for an axial O4 and thus these residues may operate as a selectivity 'dyad' for Gal with S1_20. Additionally, the residues implicated in recognition of Gal over GalNAc, E335 and R353 in BT1636 ${ }^{3 S-G a l}$ are conserved in 64 and $74 \%$ of S1_20 sequences, whilst the residue that allows the accommodation of O2 N-acetyl and activity in GalNAc (N334 in BT16223S-Gal/GalNAc) is only found in 8\% of members of this family (Extended Data Fig. 7 and Supplementary Fig. 3). This observation suggests that the majority of the S1_20 sulfatases evolved to target sulfated Gal and only a subset of this subfamily's members can actually also be active on GalNAc. Interestingly, all of the close homologs of BT1636 3 S-Gal and BT1622 3 SGal/GalNAc that share the critical specificity determinants of these proteins (Supplementary Tables 12 and 13) were isolated from mammals at body regions rich in mucins, highlighting the role of these sulfatases in accessing sulfated host glycans.

\section{Growth of sulfatase mutants on O-glycans}

The deletion strain lacking 4S-Gal/GalNAc sulfatases $(\Delta b t 3057+\Delta b t 3796)$ did not show any phenotype in CMO (Extended Data Fig. 8a), a result that is consistent with the lack of these sulfated linkages in colonic mucins (Supplementary Table 4). Unexpectedly, the deletion strains lacking the identified $6 S-G a l / G a I N A c$ sulfatases $(\Delta b t 1624+\Delta b t 3109+\Delta b t 4631)$ and 6 S-GlcNAc sulfatases $(\Delta b t 1628+\Delta b t 3051+$ $\Delta b t 3177)$ also did not show any growth defect on cMOs (Extended Data Fig. 8a). Analysis of $\mathrm{cMO}$ by mass spectrometry showed that this substrate contains a low abundance of $6 S-G a l$ but a relatively high abundance of 6 S-GIcNAc, especially in shorter structures (Supplementary Table 4). Although the low abundance of O6sulfated Gal could explain the lack of phenotype of the 6S-Gal/GalNAc sulfatase deficient strain, the lack of effect in the $\triangle 6 S-G / c N A c$ mutant in $\mathrm{cMO}$ was unexpected (Extended Data Fig. 8a). Due to the limitations of the mass spectrometry technique it is not possible to analyse sulfation in longer oligos, making the real complexity of glycans found in colonic mucins unclear. Indeed, the lack of phenotype of $\triangle 6 S-G / c N A c$ 
mutant in $\mathrm{cMO}$ suggests that 6S-GIcNAc might not be a major terminal epitope in colonic mucins. It is also important to note that the mutant $\triangle 6 S-G / c N A c$ is the deletion of two characterized 6S-GIcNAc sulfatases active on cMO (BT1628 ${ }^{6 S-G I c N A c}$ and

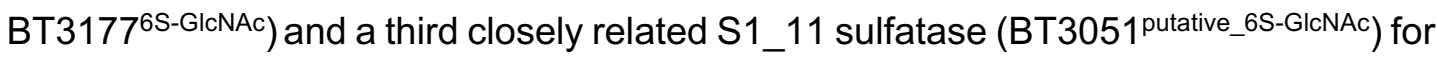
which no activity was found. This putative 6S-GICNAc sulfatase was deleted to avoid possible compensation of function after loss of BT16286S-GICNAc and BT3177 6 6S-GICNAc activities.

The deletion of previously characterized GAG-specific sulfatases ${ }^{8}(\Delta b t 1596+$ $\Delta b t 3333+\Delta b t 3349+\Delta b t 4656)$ did not result in any observable phenotype in cMO (Extended Data Fig. 8a), indicating that this substrate was not contaminated with additional endogenous host glycans. Additionally, despite some mutants exhibiting growth defects on sulfated $\mathrm{cMO}$, all of the mutants grew well on gMO and glucose (Fig. 4a and Extended Data Fig. 8a), suggesting that the phenotypes observed are dependent on the mucin source (colon) and cannot be observed utilizing mucins from other regions of the gastrointestinal tract. Together these results highlight the contribution of sulfatases in utilization of colonic mucins by the HGM.

\section{Analysis of $\Delta b t 1636^{3 S-G a l}$ culture supernatant by MS}

The analysis of the oligosaccharides present in $\Delta b t 1636^{3 S-G a l}$ culture supernatant after $96 \mathrm{~h}$ incubation revealed that the detected glycans are different from the cMO profile in the starting material (Fig. 4c, Extended Data Fig. 8b and Supplementary Table 5). We detected 114 glycans in the cMO sample, of which 39 were sulfated and fucosylated (44\% total) (Extended Data Fig. 8b) and the three most common structures (12\% total) were 6S-GIcNAc oligosaccharides (Fig. 4c and Supplementary

Table 5). In the control sample, the levels of sulfation, sialylation and fucosylation were $92 \%, 40 \%$ and $77 \%$, respectively (Supplementary Table 5). In the $4 b t 1636^{3 S-}$ Gal culture supernatant, we detected 72 glycans, of which 41 were substituted only with O-sulfate (84\% total) (Extended Data Fig. 8b). In the mutant supernatant the levels of sulfation (95\%) were similar to $\mathrm{cMO}$, however the levels of sialylation $(11 \%)$ and fucosylation (5\%) decreased substantially (Supplementary Table 5), suggesting that this mutant is not able to utilize sulfated structures and these accumulate in culture media. 
Additionally, a total of 98 of the 114 structures present in cMO were not detected in $\Delta b t 1636^{3 S-G a l}$ culture supernatant whereas in mutant supernatant, we detected 49 glycans that were not detected in the initial substrate (Supplementary Table 5). This suggests that some of the oligosaccharides present in $\mathrm{cMO}$ can support the limited growth of $\Delta b t 1636^{3 S-G a l}$ and, although this mutant is not able to utilize many sulfated cMO structures, it can still modify the glycans to create novel structures. It remains unclear which enzymes are encoded by the mutant to modify the O-glycans, but the presence of a cell surface sialidase ${ }^{9}$ can explain the decrease of sialylation levels in structures found in $\Delta b t 1636^{3 S-G a l}$ supernatant. Additionally, the presence of surface endo-acting glycoside hydrolases able to cleave O-glycans into shorter oligosaccharides ${ }^{10}$ can also contribute to new glycan structures in the mutant culture supernatant. Together these results show that $\Delta b t 1636^{3 S-G a l}$ is not able to utilize most sulfated O-glycans explaining the limited growth of this mutant in $\mathrm{cMO}$.

\section{References}

1 Arike, L. \& Hansson, G. C. The Densely O-Glycosylated MUC2 Mucin Protects the Intestine and Provides Food for the Commensal Bacteria. J Mol Biol 428, 3221-3229, doi:10.1016/j.jmb.2016.02.010 (2016).

2 Arike, L., Holmen-Larsson, J. \& Hansson, G. C. Intestinal Muc2 mucin Oglycosylation is affected by microbiota and regulated by differential expression of glycosyltranferases. Glycobiology 27, 318-328, doi:10.1093/glycob/cww134 (2017).

3 Derrien, M., Vaughan, E. E., Plugge, C. M. \& de Vos, W. M. Akkermansia muciniphila gen. nov., sp. nov., a human intestinal mucin-degrading bacterium. Int J Syst Evol Microbiol p54, 1469-1476, doi:10.1099/ijs.0.02873-0 (2004).

4 Pudlo, N. A. et al. Symbiotic Human Gut Bacteria with Variable Metabolic Priorities for Host Mucosal Glycans. MBio 6, e01282-01215, doi:10.1128/mBio.01282-15 (2015).

5 Barbeyron, T. et al. Matching the Diversity of Sulfated Biomolecules: Creation of a Classification Database for Sulfatases Reflecting Their Substrate Specificity. PLoS One 11, e0164846, doi:10.1371/journal.pone.0164846 (2016).

6 Hettle, A. G. et al. The Molecular Basis of Polysaccharide Sulfatase Activity and a Nomenclature for Catalytic Subsites in this Class of Enzyme. Structure 26, 747-758 e744, doi:10.1016/j.str.2018.03.012 (2018).

7 Cartmell, A. et al. How members of the human gut microbiota overcome the sulfation problem posed by glycosaminoglycans. Proc Natl Acad Sci U S A 114, 7037-7042, doi:10.1073/pnas.1704367114 (2017).

8 Ndeh, D. et al. Metabolism of multiple glycosaminoglycans by Bacteroides thetaiotaomicron is orchestrated by a versatile core genetic locus. Nat Commun 11, 646, doi:10.1038/s41467-020-14509-4 (2020). 
9 Briliute, J. et al. Complex N-glycan breakdown by gut Bacteroides involves an_SI. Nat Microbiol 4, 1571-1581, doi:10.1038/s41564-019-0466-x (2019).

10 Crouch, L. I. et al. Prominent members of the human gut microbiota express endo-acting O-glycanases to initiate mucin breakdown. Nat Commun 11, 4017, doi:10.1038/s41467-020-17847-5 (2020). 


\section{Figures}

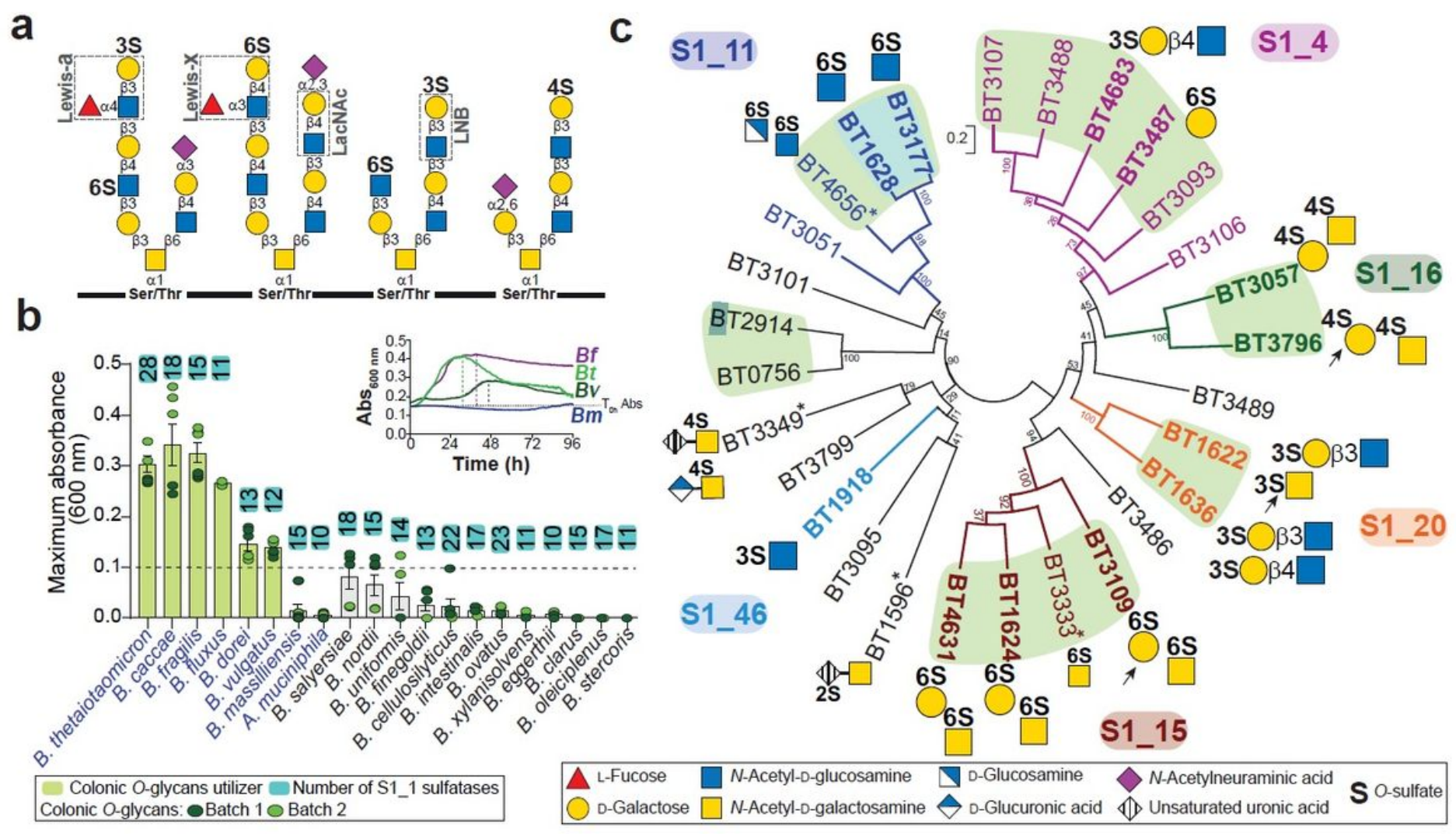

\section{Figure 1}

Bacterial growth on colonic mucin and Bt sulfatase activities. a, Schematic representation of mucin 0glycans and relevant terminal epitopes (dashed boxes). Sugars are shown according to the Symbol Nomenclature for Glycan system60. b, Growth of Bacteroides type strains and Akkermansia muciniphila on colonic mucin $\mathrm{O}$ glycans ( $\mathrm{CMO}$ ) and number of respective encoded S1 sulfatases. The bars represent the average of two independent experiments with different batches of cMO. Bacterial species able to utilize gastric mucin glycans are highlighted in blue. Maximum absorbance is the difference of the maximum absorbance value (Abs600nm) for each culture and the initial absorbance at time 0 (TOh). Graphic shows the example of growth curves for B. fragilis (Bf), B. thetaiotaomicron (Bt), B. vulgatus (Bv) and B. massilliensis (Bm). C, Phylogeny of Bt sulfatases showing the $28 \mathrm{~S} 1$ sulfatases and their respective substrates where known, including this study. Enzymes are color coded according the respective subfamilies with sulfatases characterized in this study highlighted in bold. * indicates sulfatase activity previously characterized and arrows point the substrate preferentially targeted by the respective enzyme. Sulfatases on a shared branch that share more than $86 \%$ and $39-58 \%$ of sequence identity are highlighted in blue and green background, respectively. Data from biological replicates $n=3$ to 6 and error bars denote s.e.m.. 


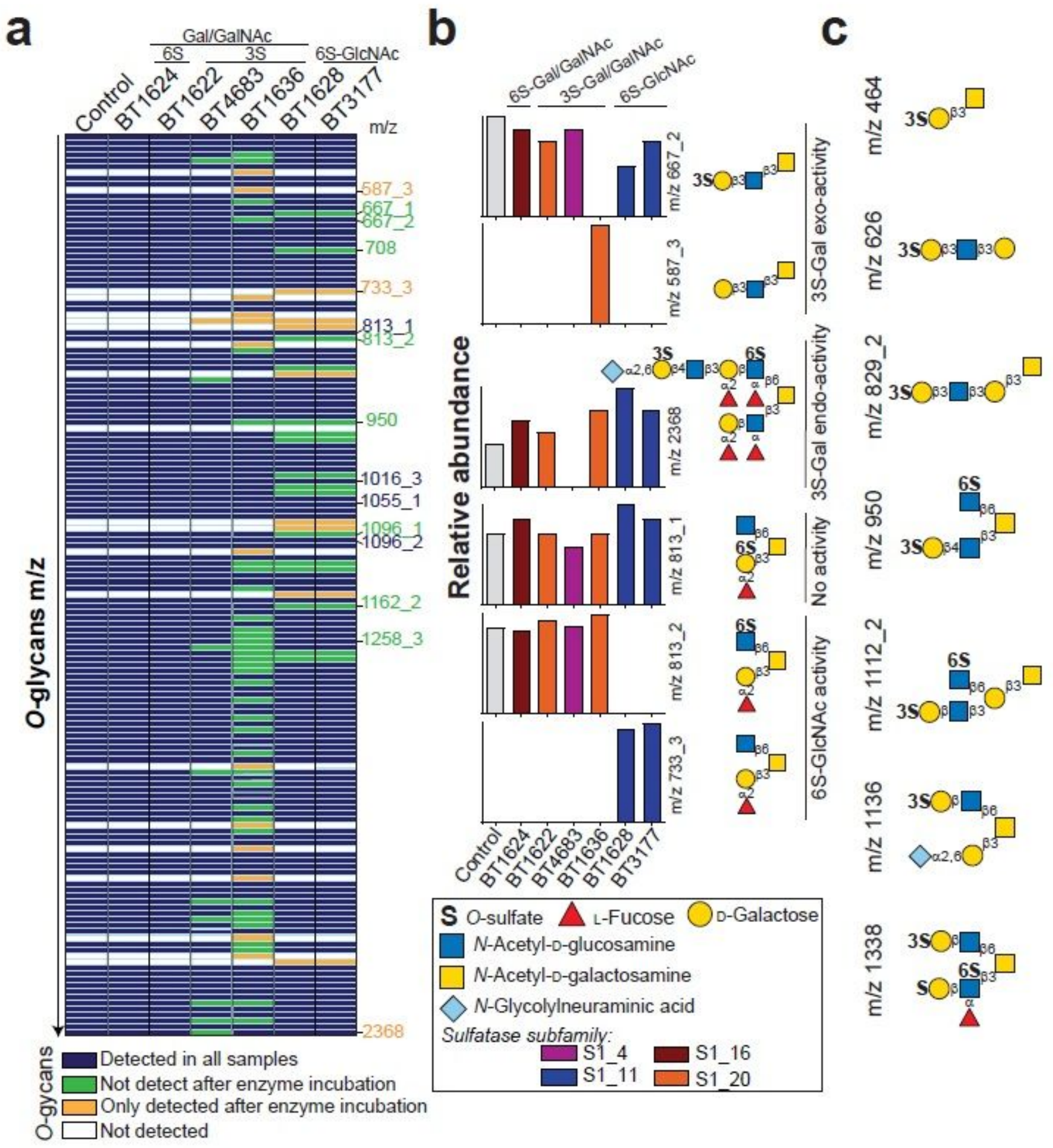

Figure 2

Activity of Bt sulfatases on colonic mucin 0-glycans. a, Representation of O-glycans detected by mass spectrometry in $\mathrm{CMO}$ (control) and after sulfatase treatment from the lower (top) to the higher (bottom) mass range. b, Relative abundance and putative structures for the specific $\mathrm{m} / \mathrm{z}$ shown in panel $a$. Remaining structures are shown in Extended figure 5a. c, Schematic representation of the putative structures that were not detected after treatment with BT16363S-Gal. 

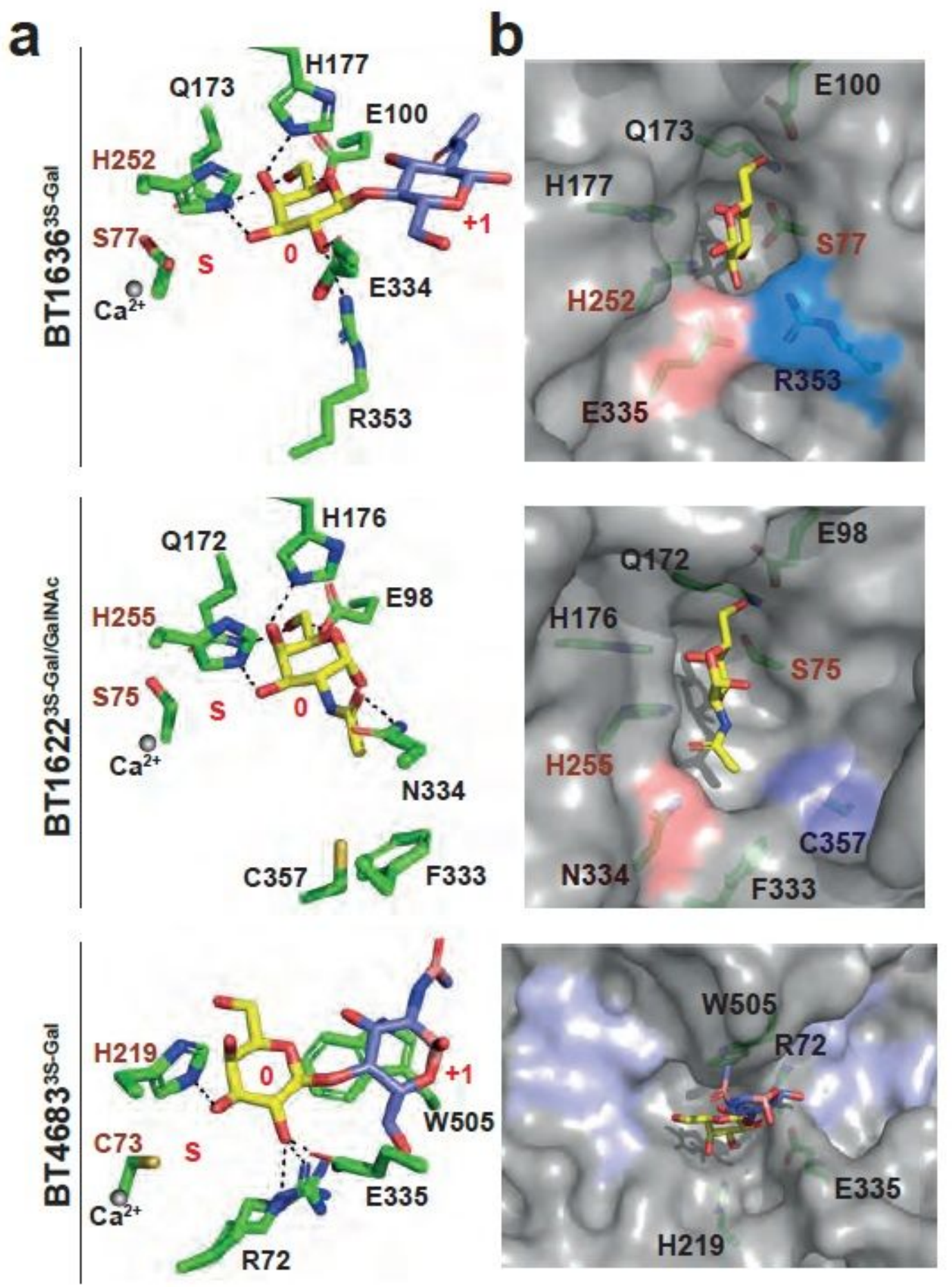

\section{Figure 3}

Crystal structures of 3S-Gal/GalNAc sulfatases. a, Schematic representation of the residues interacting with targeted sugars, including the putative catalytic residues (in dark red), the calcium ion (grey sphere) and subsites S, 0 and +1 highlighted in red. BT16363S-Gal and BT46833S-Gal in complex with LacNAc (D-Gal-b1,4-D-GlcNAc) and BT16223S-683 Gal/GalNAc in complex with GalNAc. b, Surface representation of the active pocket. The equivalent Gal/GalNAc specificity residues in BT16363S-Gal and BT46833S-Gal 
are highlighted in red and blue. The open active site of BT46833S-Gal is highlighted in purple. In all structures the amino acids and ligands are represented as stick.

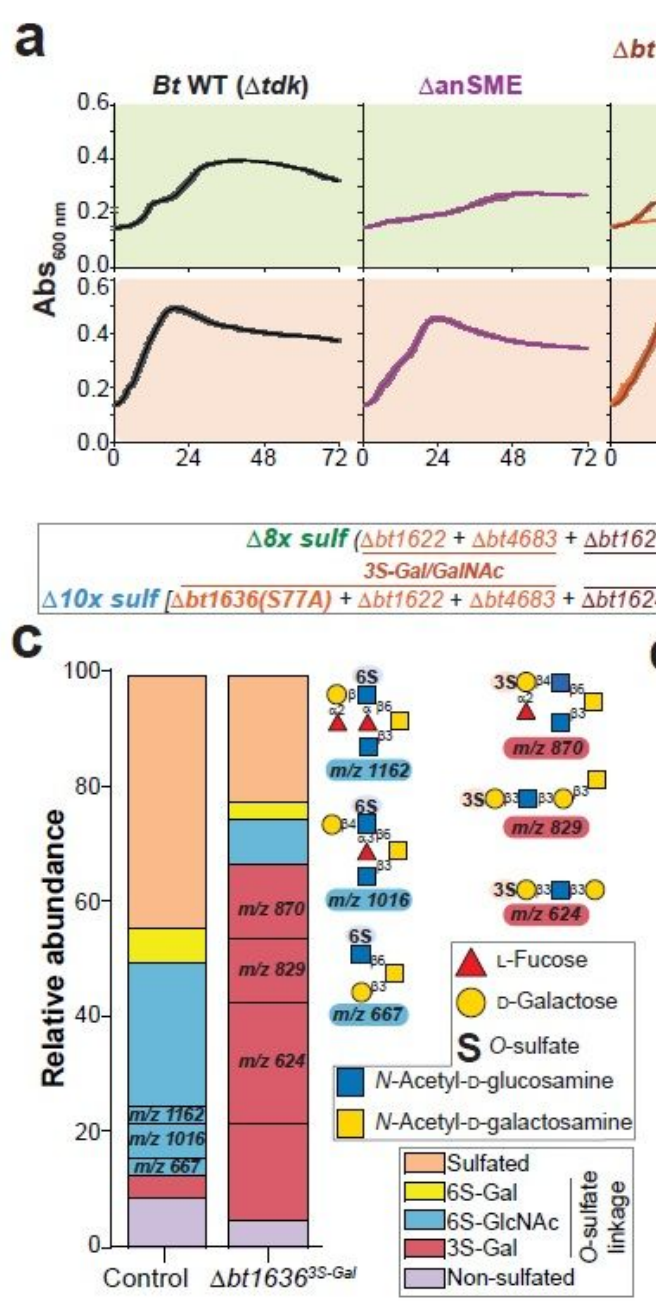

$\Delta$ bt1636::bt16363s-Gal

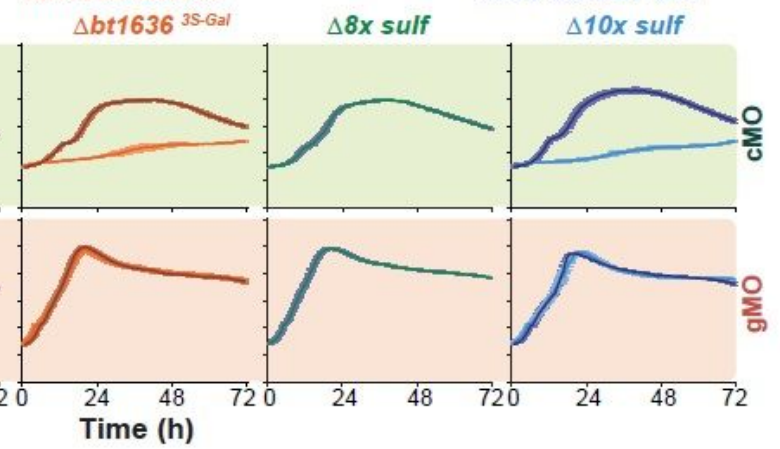

$\Delta 10 x$ sulf::bt1636 $35-G a l$
$6 S-G a l / G a l N A c$

4S-Gal

$+\Delta b t 3109+\Delta b t 4631+\Delta b t 1628+\Delta b t 3177+\Delta b t 3057+\Delta b t 3796]$

b

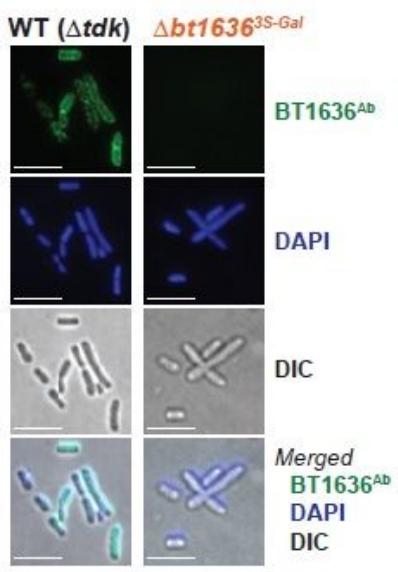

d

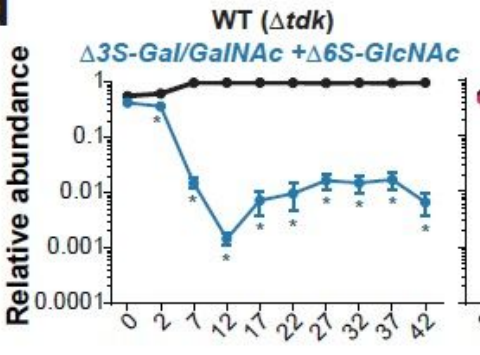

WT ( $\Delta t d k)$ $\triangle 3 S-G a l / G a I N A c$

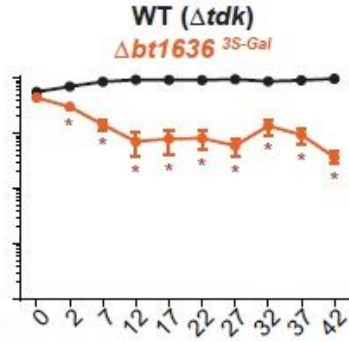
Days

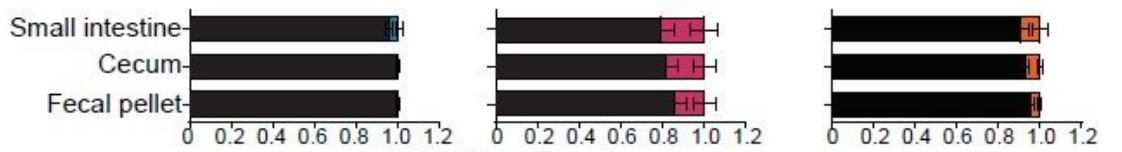

Relative abundance

$\Delta 3 S-G a l / G a l N A c+\Delta 6 S-G I c N A c[\Delta b t 1636(S 77 A)+\Delta b t 1622+\Delta b t 4683+\Delta b t 1628+\Delta b t 3177+\Delta b t 3051]$ $\Delta 3 S-G a l / G a l N A c[\Delta b t 1636(S 77 A)+\Delta b t 1622+\Delta b t 4683]$

\section{Figure 4}

BT16363S-Gal activity is required for the utilization of $\mathrm{CMO}$ and competitive fitness in vivo. a, Growth of Bt wild-type Dtdk (WT), different sulfatase gene-deletion mutants (named "DbtXXXX") and strains complemented with bt16363S-Gal on colonic or gastric mucin 0-glycans (cMO and gMO, respectively) (line represents the average of biological replicates $(n=3)$ and error bars denote s.e.m.) b, Immunofluorescent and differential interference contrast (DIC) microscopy of Bt WT and sulfatase mutant staining with polyclonal antibody (Ab) against BT16363S-Gal (green) and DNA staining with DAPI (blue). c, Relative abundance of different O-glycans detected by mass spectrometry in Dbt16363SGal culture supernatant or $\mathrm{cMO}$ in minimal media without bacteria (control), after $96 \mathrm{~h}$ in anaerobic conditions. The mass and associated structure of the 3 more abundant glycans in both samples are shown. $d$, in vivo competitions in gnotobiotic mice $(n=5-9$ separate mice from two separate experiments, except competition of the D6S-GICNAc mutant that showed no defect in one experiment) fed fiber-free diet and inoculated with WT and mutants. The fecal relative abundance of each strain was determined 
along the time course and in small intestine and cecum at day 42 (experimental endpoint). The relative abundance in each mouse is represented in the respective light colour. The error bars denote s.e.m. Significant differences between wild-type and mutant strain were compared at each time point using student's t-test (paired, one tail) and * indicates sample days in which the mutant was significantly different $(p<0.01)$ from the wild-type.

\section{Supplementary Files}

This is a list of supplementary files associated with this preprint. Click to download.

- SupplementaryTablesandFigures.pdf

- SupFigure2.pdf

- SupFigure3.pdf

- SupplementaryTable4.xlsx

- SupplementaryTable5.xlsx 